bleiben somit die Möglichkeiten $\mathrm{H}_{2} \mathrm{O}, \mathrm{H}_{2} \mathrm{O}^{+}, \mathrm{O}_{2} \mathrm{H}$ oder $\mathrm{O}_{2} \mathrm{H}^{+}$. Da in der 50:50-Mischung kein drittes neues Spektrum auftritt, wie es für die HDO-Konfiguration zu erwarten wäre, ist anzunehmen, daß in dem Träger nur ein H-Atom vorhanden ist. Da weiter in der Aufnahme mit $\mathrm{D}_{2} \mathrm{O}^{18}\left(10 \% \mathrm{O}^{18}\right)$ ebenfalls keine neuen Spektren gefunden wurden, ist wahrscheinlich, daß im Träger nicht mehr als zwei O-Atome enthalten sind. Eine versuchsweise Zuordnung des neuen Spektrums zu $\mathrm{O}_{2} \mathrm{H}$ oder $\mathrm{O}_{2} \mathrm{H}^{+}$erscheint somit plausibel. Das in der $\mathrm{H}_{2} \mathrm{O}$-Entladung beobachtete Spektrum wurde ausgemessen, aber kein Versuch einer Analyse gemacht.
Mein Dank gilt vor allem meinem verehrten Lehrer, Herrn Professor SchüLer, für sein stets förderndes Interesse am Fortgang dieser Arbeit und für viele wertvolle Ratschläge. Die Untersuchung im Schumans-Gebiet wurde während eines Aufenthalts an der Physikalischen Anstalt der Universität Basel im Laboratorium von Herrn Professor E. Miescher durchgeführt, dem ich für seine liebenswürdige Gastfreundschaft, sein dauerndes Interesse und viele Diskussionen zu großem Dank verpflichtet bin. Mein Dank gilt weiter dem Direktor der Physikalisch-Chemischen Anstalt der Universität Basel, Herrn Professor Kuns, der eine größere Menge $\mathrm{D}_{2} \mathrm{O}^{16}$ sowie $\mathrm{D}_{2} \mathrm{O}^{18}$ großzügig zur Verfügung gestellt hat.

Die Arbeit wurde mit dankenswerter Unterstützung der Deutschen Forschungsgemeinschaft durchgeführt.

\title{
Dichteste Atompackung und Sdhichtpaket-Bildung in einatomigen Metallschmelzen
}

\author{
Von H. Richter, G. Breitling und F. Herre \\ Aus dem Röntgeninstitut der Technischen Hochschule Stuttgart und dem Institut für Metallphysik \\ am Max-Planck-Institut für Metallforschung, Stuttgart \\ (Z. Naturforschg. 12 a, 896-925 [1957]; eingegangen am 14. Juni 1957)
}

\begin{abstract}
Eingehende Betrachtungen der Intensitätskurven einatomiger Metallschmelzen zeigen, daß die Lagen der Maxima im wesentlichen durch den kürzesten Atomabstand $r_{1}$ und damit durch eine einzige Interferenzfunktion $\sin x_{1} / x_{1}$ festgelegt werden. Bei Kenntnis von $r_{1}$ bzw. des Atomradius $a_{\mathrm{K}}{ }^{*}$ läßt sich daher die Lage des ersten Maximums nach der Gleichung $\sin \vartheta_{\mathrm{I}} / \lambda=0,6147 \cdot 1 / r_{1}=0,307 \cdot 1 / a_{\mathrm{K}}$ " berechnen. Trägt man die aus dem Experiment erhaltenen $\sin \vartheta_{\mathrm{I}} / \lambda$-Werte über dem Atomradius $a_{\mathrm{K}} *$ für Zwölfer-Koordination als Abszisse auf, so sieht man, daß sämtliche $\sin \vartheta_{\mathrm{I}} / \lambda$-Werte der bisher untersuchten Metallschmelzen praktisch auf die $\sin \vartheta_{\mathrm{I}} / \lambda \cdot a_{12}{ }^{*}$-Kurve (gleichseitige Hyperbel) fallen, d. h. in den Metallschmelzen liegt eine Zwölfer-Koordination, also eine dichteste Packung der Atome nach Art des statistischen Kugelmodells vor.

Weiter zeigt die Diskussion der Streukurven geschmolzener Metalle, daß die dichteste Atompackung besonders in der Nähe des Schmelzpunktes sehr häufig von einer Paket-Struktur, z. Tl. mit Flächengitter-Charakter, überlagert ist. Mit steigender Temperatur verliert sich diese SchichtpaketBildung.
\end{abstract}

\section{Diskussion der Atomverteilungskurve von geschmolzenem Au und $\mathrm{Na}$}

Die Deutung der Atomverteilungskurven einatomiger geschmolzener Metalle in Verbindung mit den zugehörigen Streukurven gestattet nach Richter, Breitling und Herre ${ }^{1}$ einige grundlegende Aussagen über die Atomanordnung in einfachen Metallschmelzen zu machen. Es seien zunächst die kürzesten Atomabstände $r$ und die zugehörigen Atomzahlen $N$ im Au- und Na-Gitter (Zwölfer- und Achter-Koordination) betrachtet.

${ }^{1}$ H. Richter, G. Breitling u. F. Herre, Naturw.44, 109 [1957].

\section{Au-Gitter}

I. Koord. $r_{1}=\frac{a_{\mathrm{K}}}{2} \sqrt{2}=2,88 \AA\left(N_{1}=12\right)$,

II. Koord. $r_{2}=r_{1} \sqrt{2}=a_{\mathrm{K}}=4,07 \AA\left(N_{2}=6\right)$.

\section{Na-Gitter}

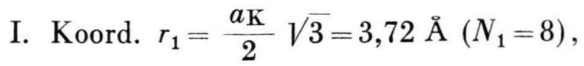

II. Koord. $r_{2}=\frac{2}{3} r_{1} \sqrt{3}=a_{\mathrm{K}}=4,30 \AA\left(N_{2}=6\right)$.

Läge in der Schmelze von Au bzw. Na eine Atomanordnung wie im zugehörigen Gitter vor, so müßte man die beiden Koordinationen I und II vielleicht schon mit $\mathrm{CuK}_{\alpha}$-Strahlung auflösen können, oder aber die Lage des ersten Maximums in den Atom- 


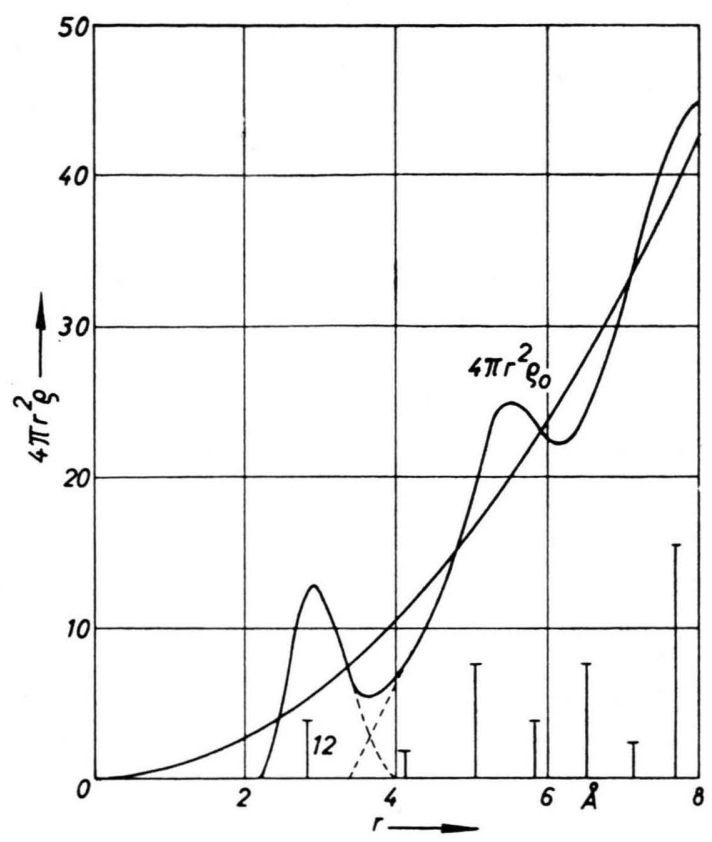

Abb. 1. Atomverteilungskurve von geschmolzenem Au. $\mathrm{I}=$ Atomlagen im Gitter.

verteilungskurven müßte durch Superposition, am ehesten bei $\mathrm{Na}$, nach größeren $r$-Werten verschoben sein. In diesem Falle hätte man zu erwarten

$$
\begin{aligned}
& (r)_{\mathrm{Au}}=3,28 \AA \text { mit } \quad N=18 \text { Atomen, } \\
& (r)_{\mathrm{Na}}=3,97 \AA \text { mit } \quad N=14 \text { Atomen; }
\end{aligned}
$$

man findet aber

$$
\begin{aligned}
& \left(r_{1}\right)_{\mathrm{Au}}=2,91 \AA \text { mit } N_{1} \approx 11 \text { Atomen, } \\
& \left(r_{1}\right)_{\mathrm{Na}}=3,83 \AA \text { mit } N_{1} \approx 10 \text { Atomen, }
\end{aligned}
$$

d. h. das erste Maximum wird sowohl bei Au als auch bei Na praktisch beim kürzesten Atomabstand ${ }^{2}$ des zugehörigen Gitters erhalten (vgl. auch die Abb. 1 und 2).

Die zweite Atomgruppe, im Falle des Au-Gitters mit $r_{2}=4,07 \AA$ und $N_{2}=6$ Atomen, zeichnet sich in der Atomverteilungskurve nicht ab, weder direkt noch in einer Verschiebung des ersten Maximums nach größeren $r$-Werten. Das zweite Maximum in Abb. 1 bei $r \approx 5,50 \AA$ (vgl. Hendus ${ }^{3}$ und Glocker ${ }^{4}$ ) kommt durch Uberlagerung der Atomgruppen des Au-Gitters $r_{3}=r_{1} \sqrt{3}=4,98 \AA$ und $r_{4}=2 r_{1}=5,76 \AA$

2 Die allgemein bei den Alkali-Metallen beobachtete Vergrößerung des kürzesten Gitterabstandes beim Schmelzvorgang hat in der veränderten Packungsdichte ihre Ursache.

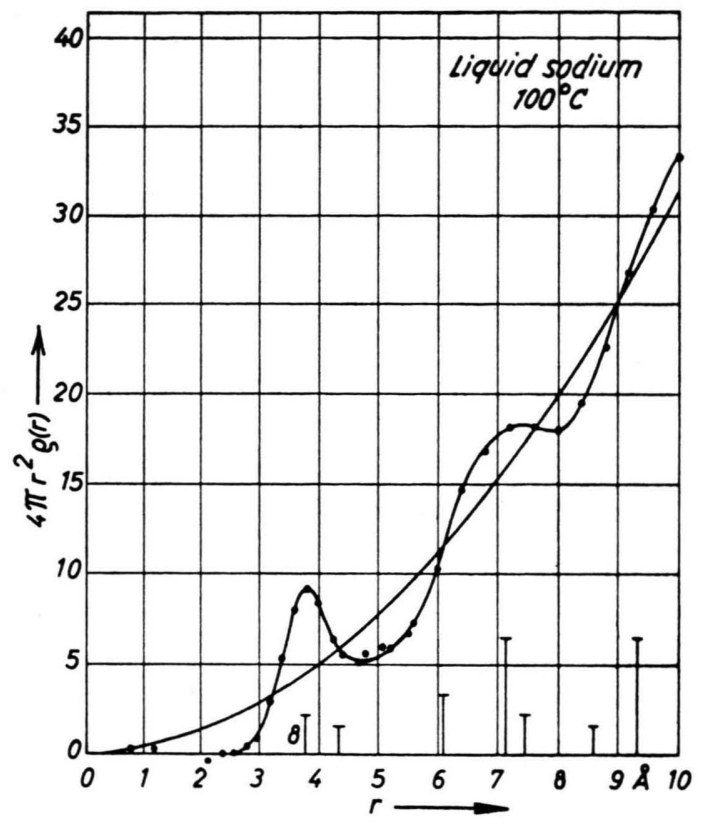

Abb. 2. Atomverteilungskurve von geschmolzenem Na. $\mathrm{I}=$ Atomlagen im Gitter.

zustande, d. h. die Atome dieser Koordinationen streuen stark um die bevorzugten Lagen. Von den Atomgruppen bei $r_{3}$ mit $N_{3}=24$ und $r_{4}$ mit $N_{4}=12$ liegen jeweils 6 Atome, zusammen mit 6 Atomen der ersten Koordination, in der (111)-Ebene des Au-Gitters; lediglich diese Atome heben sich in der Verteilungskurve der Abb. 1 ab. Dieser Befund bedeutet, daß in flüssigem $\mathrm{Au}$ - was die geordneten Bereiche in der Schmelze anlangt - offensichtlich (111)-Ebenen mit geringer Ordnung bei z. Tl. beliebiger Parallel-Lagerung, zu Schichtpaketen aneinander gefügt, vorhanden sind. Diese Schichtpakete kommen nicht als diskrete Einzel-Teilchen vor, sondern sind in steter Verbindung miteinander und mit der Kontinuität der Raumerfüllung des statistischen Kugelmodells.

Bei den Alkali-Metallen Li, Na, K usf., die raumzentriert-kubisch kristallisieren, liegen in der Schmelze die Verhältnisse völlig analog. Die zweite Atomgruppe des Gitters mit $r_{2}=\frac{2}{3} r_{1} \sqrt{3}=a_{\mathrm{K}}$ und mit $N_{2}=6$ Atomen ist in der Verteilungskurve ebenfalls nicht zu beobachten (vgl. Abb. 2).

3 H. Hendus, Z. Naturforschg. 2 a, 505 [1947].

4 R. Glocker, Ergebn. exakt. Naturw. 22, 186 [1949]. 


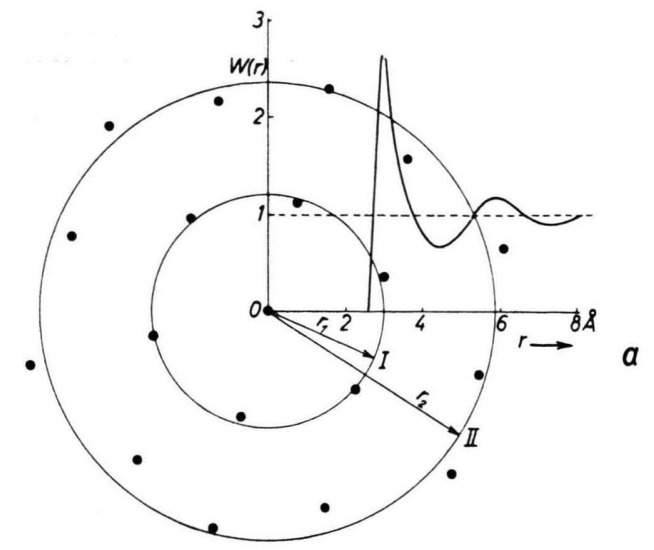

Um die Atomverteilung in einatomigen Metallschmelzen zu beschreiben, braucht man demnach zwei Modelle:

1. das statistische Kugelmodell und

2. das Schichtpaketmodell.

Das statistische Kugelmodell sei an Hand der Abb. 3 a betrachtet. Die Ordinaten-Werte der eingezeichneten Verteilungskurve über dem Atomabstand $r$ als Abszisse sind ein $\mathrm{Maß}$ für die Häufigkeit der Atomlagen. Danach wird der kürzeste $\mathrm{Ab}$ standswert $r_{1}=3,0 \AA$ von den Atomen bevorzugt eingenommen, ein weiterer liegt bei $r_{2} \approx 5,9 \AA$, nur sind hier die Streuungen der Atomlagen erheblich stärker. Für große $r$-Werte geht die Atomverteilung allmählich in eine gleichmäßige über. Diese Atomanordnung in einer Flüssigkeit läßt sich durch eine Verteilung der Atome auf Kugelschalen (statistisches Kugelmodell) darstellen. Hierzu schlägt man um das Ausgangsatom 0 eine Kugel vom Radius $r_{1}$. Man sieht, daß die Atome der I. Koordination mit nur sehr geringen Streuungen auf der Kugeloberfläche liegen. Macht man den Radius der Kugel gleich dem zweiten bevorzugten Abstand (II. Koordination), dann sind die Streuungen ganz beträchtlich stärker. Für noch größere Abstandswerte sind kaum noch weitere Häufungsstellen der Atomlagen zu beobachten. Dabei ist für jedes Atom als Ausgangsatom die Verteilung stets die gleiche. Beim statistischen Kugelmodell ist der kürzeste Atomabstand dominierend, da die weiteren bevorzugten Abstände wegen zu großer Streuung der Atomlagen stark zurücktreten.

Zur Diskussion des Begriffes „Schichtpaket“ seien die Abb. 3 b, c, d betrachtet. In Abb. 3 b ist ein Raumgitter in schematischer Darstellung wiedergegeben. Hier treten bei geeigneter Kristall-Lage die

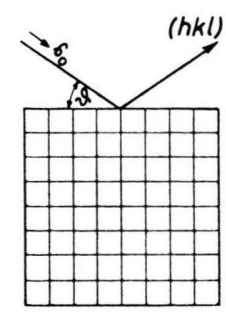

$b$

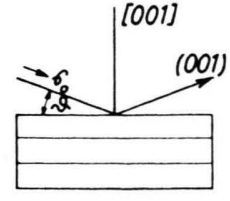

C

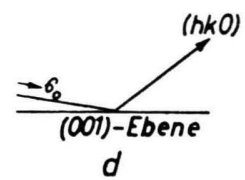

Abb. 3. Strukturmodelle in schematischer Darstellung. a) Statistisches Kugelmodell, b) Raumgitter, c) Schichtpaket, d) Flächengitter.

Raumgitter-Interferenzen ( $h k l)$ entsprechend den Intensitätsgesetzen auf. In Abb. $3 \mathrm{c}$ ist das Raumgitter bis auf wenige Schichtlagen zu einem „Schichtpaket" abgebaut. Jetzt tritt vorwiegend die (001) Interferenz auf, alle weiteren Raumgitter-Interferenzen $(h k l)$ sind wegen zu geringer Anzahl von Streuzentren in den betreffenden Netzebenen stark verbreitert und geschwächt. Bei regelloser Parallel-Lagerung der Einzel-Schichten innerhalb des Schichtpaketes verschwinden die Raumgitter-Interferenzen $(h k l)$ bis auf die (001)-Interferenz; zudem verhält sich jetzt das Schichtpaket wie eine einzelne Netzebene, d. h. es zeigt auch „Flächengitter-Charakter". Liegt wie in Abb. $3 \mathrm{~d}$ nur eine einzelne Netzebene, ein „Flächengitter" vor, dann treten lediglich die Flächen " ${ }^{++r}$. Interferenzen $(h k 0)$ auf (vgl. Anm. ${ }^{50}$ ).

Es ist möglich ${ }^{5}$, die Atomverteilungskurve für geordnete Bereiche durch Fourier-Umkehr der berechneten Streukurve zu erhalten. So sind in den Abb. 4 a, b, c drei Verteilungskurven für Au wiedergegeben; und zwar in Abb. 4 a für eine Einzelschicht mit insgesamt $N^{*}=13$ Atomen [(111)-Ebene des flächenzentriert-kubischen Au-Gitters], in Abb. 4b für drei übereinander gelagerte (111)-Ebenen mit $N^{*}=37$ Atomen und einer Schichtanordnung wie im Au-Gitter und schließlich in Abb. $4 \mathrm{c}$ für eine schalenartige Anordnung von $N_{1}=12$ Atomen um ein Aus gangsatom (statistisches Kugelmodell einer einatomigen Flüssigkeit). In allen drei Fällen liegt eine dichteste Anordnung der Atome vor, ferner beträgt der kürzeste Atomabstand $r_{1}=2,91 \AA$ und die Integrationslänge wie beim Experiment

$$
s_{0}=4 \pi(\sin \vartheta / \lambda)_{\max .}=7,5 .
$$

Das deutliche Maximum bei $r \approx 4,0 \AA$ in den Abb. 4 a

5 H. Richter, G. Breitling u. F. Herre, Naturw. 42, 151 [1955]. 


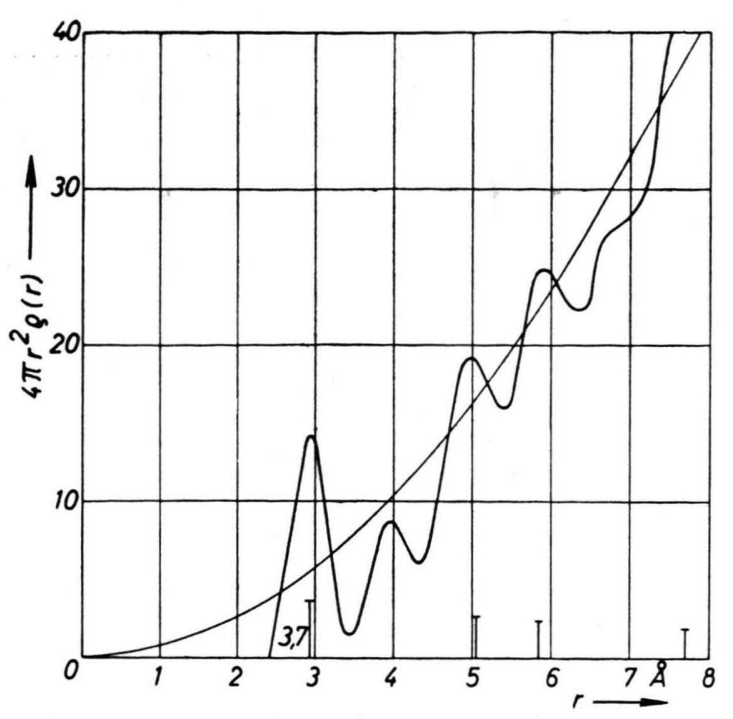

Abb. 4. Berechnete Atomverteilungskurven für Au. $\left(r_{1}=2,91 \AA\right.$ And $\left.s_{\sigma}=4 \pi(\sin \vartheta / \lambda)_{\max .}=7,5\right)$.

a) Einzelschicht mit $N^{*}=13$ Atomen,

und c kommt durch das Zusammenspiel der Nebenmaxima der berücksichtigten Atomabstände zustande; es ist also nicht reell. In Abb. $4 \mathrm{~b}$ ist dieses Maximum durch die zweite Atomgruppe $r_{2}=r_{1} \sqrt{2}=4,12 \AA$ mit $N_{2}=6$ Atomen beträchtlich angehoben und, wie verlangt, geringfügig nach größeren $r$-Werten verlagert. Abb. $4 \mathrm{c}$ zeigt nur ein ausgeprägtes Maximum, da das ihr zugrunde gelegte Modell durch einen einzigen Atomabstand charakterisiert ist. In allen drei Modellen besitzen die Atome ganz bestimmte, diskrete Abstände voneinander; im Gegensatz dazu sind in den Metallschmelzen z. Tl. beträchtliche Streuungen der Atomlagen vorhanden, die mit dem Abstand vom Zentralatom rasch anwachsen (vgl. Kratky ${ }^{6}$ ). Diese Streuungen der Atome haben eine mit dem Abstand zunehmende Verbreiterung der Maxima bei abnehmender Höhe zur Folge. Eine solche Verbreiterung bringt in Abb. 4 a das Nebenmaximum bei $r \approx 4,0 \AA$ praktisch zum Verschwinden und überlagert die Maxima der beiden Atomgruppen bei $r_{3}=5,04 \AA$ und $r_{4}=5,82 \AA$ in Übereinstimmung mit dem Experiment zu einem Maximum bei $r \approx 5,50 \AA$. In Abb. $4 \mathrm{~b}$ müßten dagegen die Maxima bei $r_{2}=4,12 \AA$ und $r_{3}=5,04 \AA$ trotz ihrer Verbreiterung noch deutlich zu erkennen sein. Das Maximum bei $r_{2}=4,12 \AA$ kommt allerdings dann zum Ver-

\footnotetext{
${ }^{6}$ O. Kratky, Phys. Z. 34, 482 [1933].
}

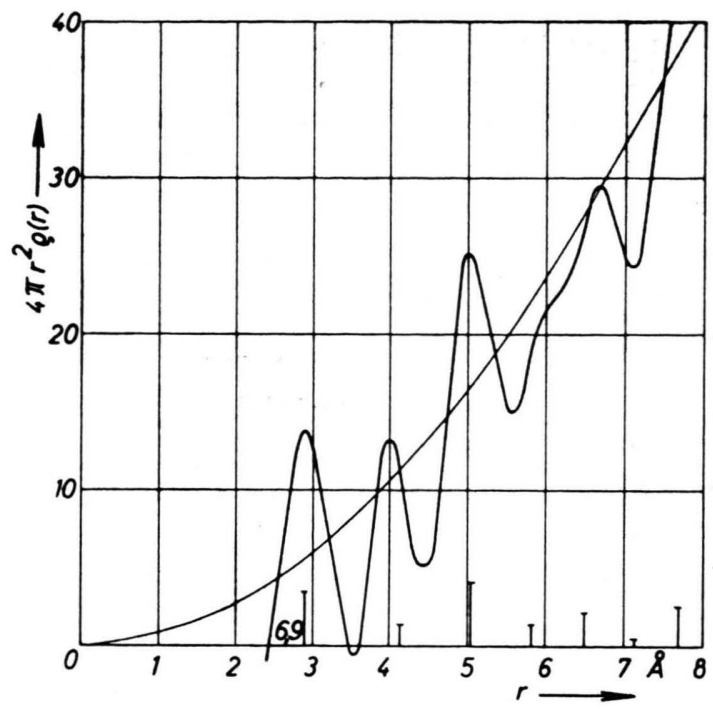

b) Drei Schichten mit $N^{*}=37$ Atomen,

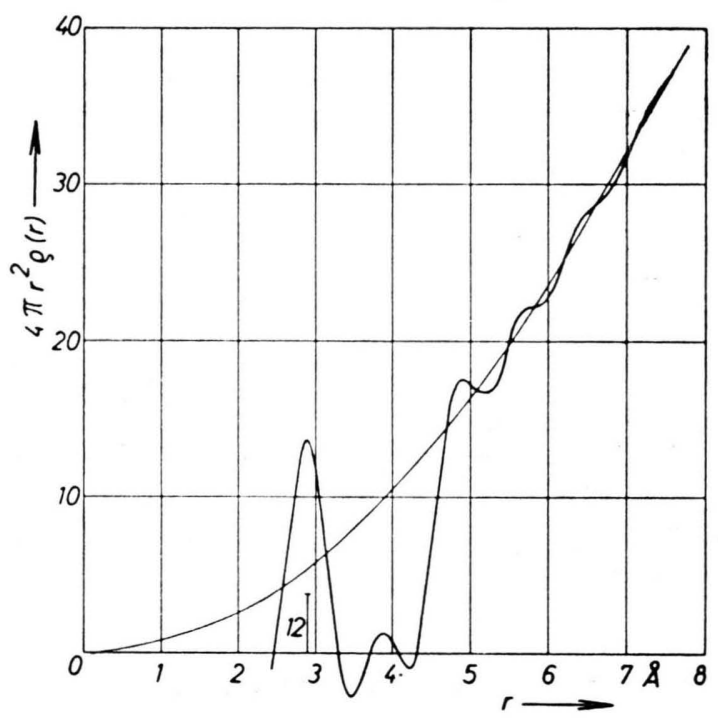

c) Statistisches Kugelmodell mit $N_{1}=12$ Atomen.

schwinden, wenn man annimmt, daß die Streuung der Atome um ihre Lagen durch die Breite des ersten Maximums in Abb. 1 gegeben wäre. Infolge des Abbrucheffektes (vgl. Richter, Breitling und Herre ${ }^{7}$ ) trifft aber diese Annahme nicht zu. So ist wegen der fehlenden Übereinstimmung der Atomverteilungskurve in Abb. $4 \mathrm{~b}$ mit dem experimentellen Befund in Abb. 1 die gitterähnliche An-

7 H. Richter, G. Breitling u. F. Herre, l. c. ${ }^{5}$; Z. angew. Phys. 8, 433 [1956]. 
ordnung der Atome in flüssigem Au auszuschließen, d. h. die Au-Schmelze ist nicht als ein gleichmäßig verwackeltes Raumgitter aufzufassen. Ebensowenig scheint nach Abb. 4c das Kugelmodell die Verhältnisse in Metallschmelzen wiederzugeben. In der Nähe des Schmelzpunktes gilt dieses Modell offenbar nur in Verbindung mit der Schicht- bzw. Paketstruktur, die zur Verteilungskurve in Abb. 4 a führt (vgl. Abb. 1 mit Abb. 4 a und c). Bei Temperaturen weit über dem Schmelzpunkt wird dagegen die Atomverteilung vorwiegend vom statistischen Kugelmodell bestimmt. Man sieht also, daß auch der unmittelbare Vergleich von experimenteller und berechneter Atomverteilung in flüssigem $\mathrm{Au}$ zur Vorstellung von Schichtpaketen aus (111)-Ebenen mit geringer Ordnung der Atome und mit beliebiger Parallel-Verschiebung der Schichten führt.

In der Hosemannschen Theorie des Parakristalls (vgl. Hosemann ${ }^{8}$ ) wird ein Gitter betrachtet, das sich an Stelle der üblichen drei Gittertranslationen aus drei Koordinationsstatistiken aufbaut. Durch geeignete Wahl der drei Statistiken liefert diese Theorie als Grenzfälle die Lauesche Kristall-Interferenztheorie und die Debye-Menke-Zernike-Prinssche Flüssigkeitstheorie. Weiter führt sie, ebenfalls bei geeigneter Wahl der Koordinationsstatistik, zu den obigen Schichtstrukturen, d. h. die Schichtstruktur ist auch als Spezialfall in der Hosemannschen Theorie enthalten; zudem liefert sie einen stetigen Übergang von den Raumgitter- zu den Flächengitter-Interferenzen (vgl. HosemanN ${ }^{8}$ ), d. h. von der Raumgitterzur Flächengitter-Struktur, analog den Ausführungen auf S. 898.

\section{Herkunft des kleinen Maximums in der Atomverteilungskurve geschmolzener einatomiger Metalle}

Bei einer Reihe von Elementen wie $\mathrm{Pb}, \mathrm{Na}, \mathrm{Tl}$, Cd, In, Sn u. a. führt das Vorhandensein eines kleinen Maximums in der Atomverteilungskurve, und zwar ganz in der Nähe des ersten Maximums, sehr

8 R. Hosemann, Z. Phys. 128, 1 u. 465 [1950]; 146, 588 [1956]; Naturwiss. 41, 440 [1954]; Ergebn. exakt. Naturw. 24, 142 [1951] ; Zur Struktur und Materie der Festkörper, Springer-Verlag, Berlin 1952.

- Bei flüssigem Ar tritt das kleine Maximum sogar bei Vorliegen einer Zwölfer-Koordination auf; ebenso bei geschmolzenem $\mathrm{Li}$ mit Zwölfer-Koordination, andererseits häufig zur Aufteilung der ersten Atomgruppe in $(8+4)$-Atome ${ }^{9}$. Wie die Ausführungen von Richter, Breitling und Herre ${ }^{7}$ zeigen, tritt ein solches Maximum (erstes Nebenmaximum) als Folge der endlichen Integrationslänge $s_{0}$ im Abstand $\Delta_{1}\left(s_{0}\right)=2,5 \pi / s_{0}$ vom zugehörigen Hauptmaximum auf; seine Lage kann allerdings durch das Zusammenspiel mit den Haupt- und Nebenmaxima der benachbarten Atomabstände etwas verändert sein, in seltenen Fällen kann das Nebenmaximum durch Superposition mit anderen Maxima sogar ausgelöscht sein. Der Abstand $\Delta_{1}$ des ersten Nebenmaximums von seinem Hauptmaximum in Abhängigkeit von $\sin \vartheta / \lambda$ ist in Abb. 5 dargestellt. Die ausgezogene Kurve gibt die nach oben berechnete Abhängigkeit wieder. Man sieht, wie bei Verwendung von $\mathrm{CuK}_{\alpha^{-}}$und $\mathrm{MoK}_{\alpha^{-}}$Strahlung $\left[(\sin \vartheta / \lambda)_{\max .}=0,64\right.$ und $\left.(\sin \vartheta / \lambda)_{\max .}=1,40\right]$ die experimentell erhaltenen Abstandswerte $\Delta_{1}$ geringfügig um die ausgezogene Kurve streuen, dabei zeigen sie mit der berechneten Kurve denselben Gang. In der Atomverteilungskurve der Metallschmelzen ist der kürzeste Atomabstand, zudem mit großer Atomzahl dominierend, deshalb zeichnen sich hier die Nebenmaxima besonders deutlich ab. Das kleine Maximum, das mitunter in den Verteilungskurven im Abstand $\Delta_{1}$ vom ersten Maximum auftritt, ist also nicht reell. Damit erfolgt die Aufteilung der ersten Koordination in $(8+4)$-Atome zu Unrecht. Ohne diese Aufteilung erhält man für die Koordinationszahl $N_{1}$ etwas gröBere Werte als in Tab. 1 angegeben.

FinbaK $^{10}$ glaubt das kleine Maximum durch eine ungenaue Intensitätsmessung in gewissen Teilen der Streukurve erklären zu können (vgl. auch SharraH und SмIтн $^{11}$ ). Durch eine willkürliche und nicht unbeträchtliche Änderung des Streukurven-Verlaufes eliminiert er dieses Maximum. Man sieht hieraus, $\mathrm{da} ß$ auch eine teilweise ungenaue Intensitätsmessung, allerdings von beträchtlichem Ausmaße, neue kleine Maxima in der Atomverteilungskurve hervorrufen kann, aber niemals mit so definierter Lage und Abhängigkeit wie in Abb. 5 .

fehlt es bei flüssigem $\mathrm{K}$ mit angeblich 8 Atomen in der ersten Sphäre. Das Auftreten des kleinen Maximums ist also sehr unterschiedlich.

10 Chr. Finbak, Acta Chem. Scand. 3, 1293 u. 1279 [1949]; 8, 829 [1954].

11 P.C. Sharrah u. G.P. Sмith, J. Chem. Phys. 21, 228 [1953]. 


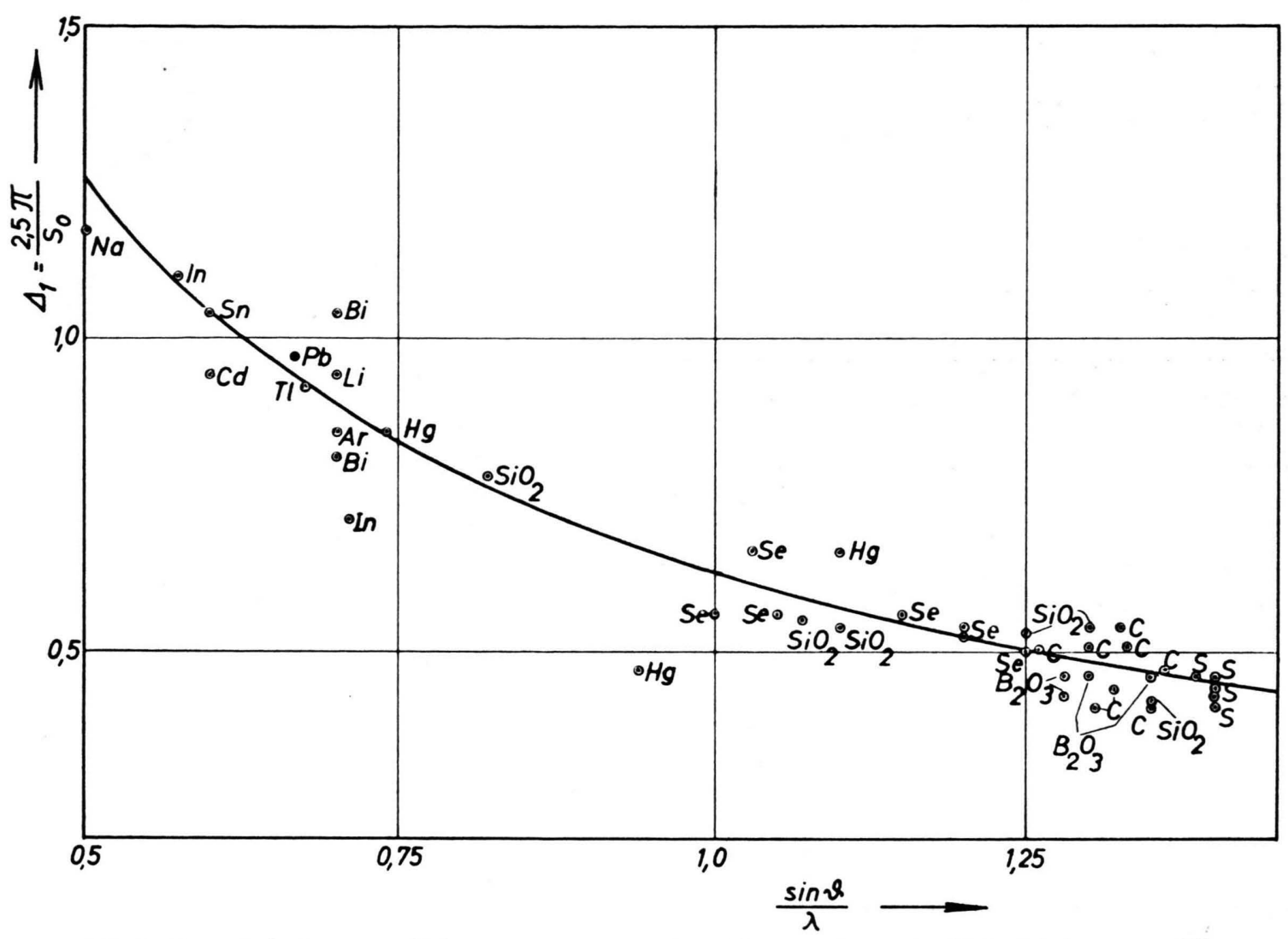

Abb. 5. Abstand $\Delta_{1}$ des ersten Nebenmaximums von seinem Hauptmaximum in Abhängigkeit von $\sin \vartheta / \lambda$.

III. Berechnung der Lage des I. Maximums der Intensitätskurve und des kürzesten Atomabstandes geschmolzener einatomiger Metalle

Wie die folgenden Ausführungen zeigen, kann man die Lagen der Maxima in der Intensitätskurve auch rechnerisch erhalten. Bei dichtester Packung der Atome nach Art des statistischen Kugelmodells seien die Maxima (Flüssigkeits-Maxima) mit I, II, III usf. bezeichnet und die experimentell erhaltenen schlechthin mit 1, 2, 3 usf.; entsprechend seien die Lagen der Flüssigkeits-Maxima durch $\sin \vartheta_{\mathrm{I}, \mathrm{II}, \mathrm{III} . . / \lambda}$ und die, wie sie vom Experiment unmittelbar geliefert werden, durch $\sin \vartheta_{1,2,3}, \ldots / \lambda$ gekennzeichnet.

\section{a) Berechnung von $\sin \vartheta_{\mathrm{I}} / \lambda$ aus der Lage des II. Maximums der Intensitätskurve}

Die Streukurve von flüssigem $\mathrm{Au}$ in Abb. 6 zeigt bezüglich der Interferenz-Lagen die gleichen Verhältniswerte wie die $\sin x / x$-Funktion hinsichtlich der Lagen der Maxima, d. h. die Interferenz-Maxima werden im wesentlichen von einem (kürzesten) $\mathrm{Ab}$ stand ${ }^{12}$ bestimmt. Sicherlich sind, wie bei der Diskussion der Atomverteilungskurve von $\mathrm{Au}$ in Abb. 1 gezeigt wurde, noch weitere Atomabstände im Spiele, doch wird durch sie der Verlauf der Intensitätskurve wegen zu starker Streuung dieser Atomlagen kaum beeinflußt. Damit liegen hier die Verhältnisse ähnlich wie beim statistischen Kugelmodell. In beiden Fällen ist der kürzeste Atomabstand dominierend. Vornehmlich durch ihn, d. h. durch ein einziges Interferenzglied, werden die Lagen der Maxima in Abb. 6 festgelegt. Damit können bei Kenntnis von $r_{1}$ die Lagen der Maxima in den Intensitätskurven einatomiger Metallschmelzen mittels der einfachen Interferenz-Funktion $\sin x_{v} / x_{v}$ mit

$$
x_{v}=k s_{v} r_{1}=2 \pi / \lambda \cdot 2 \sin \vartheta_{v} \cdot r_{1},
$$

der Bedingungsgleichung $x_{v}=\operatorname{tg} x_{v}$ und den Lösungen $x_{v}=4 \pi \cdot \sin \vartheta_{v} / \lambda \cdot r_{1}=7,725 ; 14,064 ; 20,371$;

12 Fußnote 12 auf S. 902. 
26,666; 32,956 usf. berechnet werden. Der Einfluß des Atomformfaktors wurde hier nicht berücksichtigt. Die aus $x=\operatorname{tg} x$ folgende Gleichung

$$
\sin \vartheta / \lambda=\text { const } \cdot 1 / r
$$

mit const ${ }^{13}=0,615 ; 1,119 ; 1,621 ; 2,123 ; 2,623$ usf. ist für die Diskussion der Streukurven geschmolzener Metalle von großer Bedeutung. So wurden nach ihr die Lagen der Interferenz-Maxima für geschmolzenes $\mathrm{Au}$ mit $r_{1}=2,91 \AA$ berechnet; sie sind in Abb. 6 durch die Pfeile $\bar{\downarrow}$ eingezeichnet. Andererseits ergibt sich hier die Möglichkeit, aus der Lage der Maxima den kürzesten Atomabstand $r_{1}$ in der Schmelze unmittelbar zu bestimmen. Der Wert von $r_{1}$ wird im allgemeinen durch Fourien-Analyse der Streukurve erhalten, er ist gleich dem doppelten Atomradius $a_{\mathrm{K}}{ }^{*}$. Die Maxima der Intensitätskurve kommen häufig, wie unten gezeigt wird, durch verschiedene Beugungsvorgänge zustande, und zwar durch Beugung an den dichtest gepackten Atomen der Kugelmodell-Anordnung, die zu FlüssigkeitsInterferenzen führt, sowie durch Beugung an Schichtpaketen $^{14}$ - eventuell mit dichtester Atomanordnung - , die zu Raumgitter- und Flächengitter-Interferenzen Veranlassung geben kann. Von allen Interferenz-Maxima wird das I. Flüssigkeits-Maximum der Streukurve am stärksten von den verschiedenen Beugungsvorgängen betroffen. Aus diesem Grunde ist es zweckmäßig, zur Berechnung von $r_{1}$ den $\sin \vartheta / \lambda$-Wert für das II. Maximum mit $x_{2}=14,064$ zugrunde zu legen, dessen Lage vornehmlich vom kürzesten Atomabstand des statistischen Kugelmodells bestimmt wird. Praktisch läuft die Rechnung darauf hinaus, daß man zunächst aus $\left(\sin \vartheta_{2} / \lambda\right)_{\exp }$.

12 Für die kohärent gestreute Intensität von $N^{*}$ Atomen läßt sich schreiben

$$
I(s)=N^{*} F^{2}+N^{*} F^{2} \int_{0}^{\infty} 4 \pi r^{2}\left[\varrho(r)-\varrho_{0}\right] \frac{\sin k s r}{k s r} \mathrm{~d} r .
$$

Zerlegt man die radiale Atomdichte $\varrho(r)$ in

$$
\varrho(r)=\varrho^{\prime} \delta\left(r-r_{1}\right)+\varrho_{1} S(r, R) \text { mit } r_{1}<R,
$$

wobei $\delta$ die Diracsche Funktion und $S$ eine Stufenfunktion mit den zugehörigen Grenzbedingungen bedeuten, dann gilt nach einfachem Umformen

$$
\begin{aligned}
I(s)=N^{*} F^{2} & {\left[1+N_{1} \frac{\sin k s r_{1}}{k s r_{1}}+\int_{0}^{\infty} 4 \pi r^{2}\left(\varrho_{1}-\varrho_{0}\right)\right.} \\
& \left.\cdot \frac{\sin k s r}{k s r} \mathrm{~d} r-\frac{4 \pi R \varrho_{0}}{k^{2} s^{2}}\left(\frac{\sin k s R}{k s R}-\cos k s R\right)\right]
\end{aligned}
$$

und bei $N_{1}=12, \varrho_{1} \rightarrow \varrho_{0}$ und $k s>1$ näherungsweise,

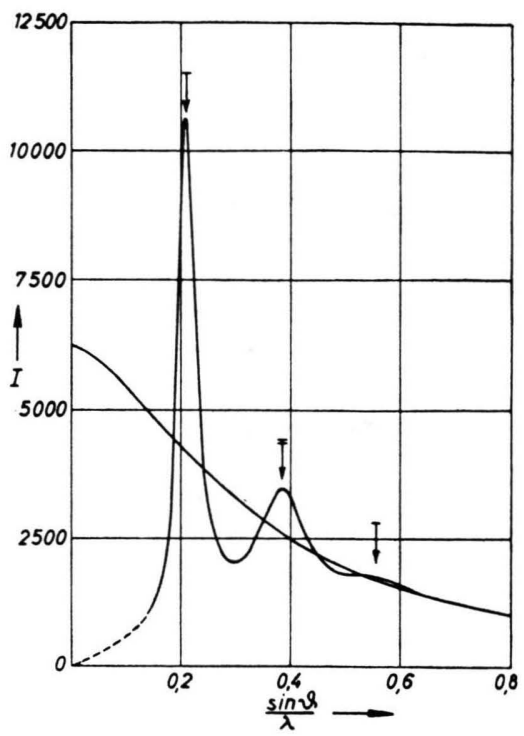

Abb. 6. Intensitätskurve von geschmolzenem Au.

$\downarrow=$ berechnete Lagen der Flüssigkeits-Maxima,

$\bar{\downarrow}=$ Berechnung zugrunde gelegte Interferenz-Lage.

$\approx \sin \vartheta_{\mathrm{II}} / \lambda=1,119 \cdot 1 / r_{1}$ die Größe $r_{1}$ berechnet, um dann aus $\sin \vartheta_{\mathrm{I}} / \lambda=0,615 \cdot 1 / r_{1}$ die tatsächliche Lage des I. Interferenz-Maximums der KugelmodellAnordnung zu erhalten. Wird die Kenntnis von $r_{1}$ nicht verlangt, dann folgt unmittelbar $\left(\sin \vartheta_{\mathrm{I}} / \lambda\right)_{\text {ber. }}$. $=0,55\left(\sin \vartheta_{2} / \lambda\right)_{\text {exp. }}$. Die aus der Lage des II. Maximums berechneten Werte ${ }^{15}$ von $\left(\sin \vartheta_{\mathrm{I}} / \lambda\right)_{\text {ber. }}$ und $\left(r_{1}\right)_{\text {ber. }}$ sind mit den entsprechenden experimentellen Daten, einschließlich der Atomzahlen $\left(N_{1}\right)_{\text {exp. }}$. in Tab. 1 zusammengestellt.

wie verlangt,

$$
I(s) \approx N^{*} F^{2}\left(1+12 \frac{\sin k s r_{1}}{k s r_{1}}\right),
$$

d. h. der gesamte Intensitätsverlauf wird, wenn die Atome für $r>R$ stark streuen, durch ein einziges Interferenzglied bestimmt.

13 Die entsprechenden Werte für die Minima sind: 0,358 ; 0,$868 ; 1,370 ; 1,872 ; 2,372$ usf.

14 Die Schichtpakete bestehen bei flächenzentriert-kubischen bzw. hexagonalen Gittern aus z. Tl. beliebig gegeneinander parallel verschobenen (111) kub.- bzw. (001) hex.-Ebenen.

15 Multipliziert man zur Schaffung gleicher Verhältnisse die Neutronen-Streukurven von $\mathrm{Pb}$ und $\mathrm{Bi}$ (Sharrah und $\mathrm{S}_{\mathrm{MITH}}{ }^{11}$ ) mit dem zugehörigen Atomformfaktor, dann erhält $\operatorname{man}\left(\sin \vartheta_{2} / \lambda\right)_{\exp .}^{\mathrm{Pb}}=0,320$ und $\left(\sin \vartheta_{2} / \lambda\right)_{\exp .}^{\mathrm{Bi}}=0,326$; hieraus folgt

$$
\left(\sin \vartheta_{\mathrm{I}} / \lambda\right)_{\text {ber. }}^{\mathrm{Pb}}=0,176 \text { und }\left(\sin \vartheta_{\mathrm{I}} / \lambda\right)_{\text {ber. }}^{\mathrm{Bi}}=0,179
$$

bzw. $\left(r_{1}\right) \mathrm{Pb}=3,49 \AA$ und $\left(r_{1}\right)_{\mathrm{Bi}}=3,43 \AA$ (vgl. Tab. 1). 


\begin{tabular}{|c|c|c|c|c|c|c|c|c|c|c|c|}
\hline \multirow[b]{2}{*}{ 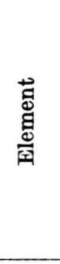 } & \multirow[b]{2}{*}{$\frac{\dot{1}}{\stackrel{0}{0}}$} & \multirow[b]{2}{*}{ 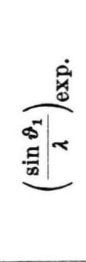 } & \multirow{2}{*}{ 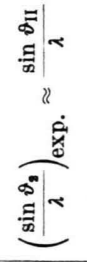 } & \multirow[b]{2}{*}{$\stackrel{\dot{\vec{d}}}{\stackrel{0}{0}}$} & \multicolumn{3}{|c|}{$\left(\frac{\sin \theta_{\mathrm{I}}}{\lambda}\right)_{\text {ber. }}$ aus den } & \multirow[b]{2}{*}{ 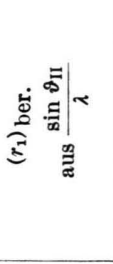 } & \multirow[b]{2}{*}{ 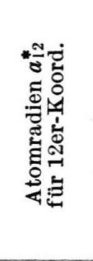 } & \multirow[b]{2}{*}{ 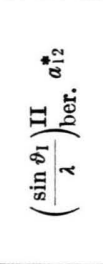 } & \multirow[b]{2}{*}{ 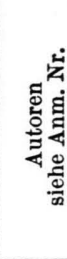 } \\
\hline & & & & & 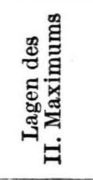 & 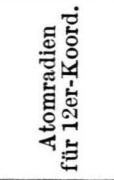 & 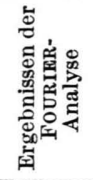 & & & & \\
\hline $\mathrm{He}$ & $\frac{-}{8,4}$ & $\begin{array}{l}0,160 \\
0,162 \\
0,164\end{array}$ & $\begin{array}{c}0,300 \\
- \\
-\end{array}$ & $\frac{\approx 3,25}{\overline{3,70}}$ & $\begin{array}{c}0,165 \\
- \\
-\end{array}$ & $\frac{-}{-}$ & $\begin{array}{c}0,188 \\
\overline{0} \\
0,165\end{array}$ & $\begin{array}{l}3,73 \\
(3,78) * \\
(3,74)\end{array}$ & - & - & $\begin{array}{l}16 \\
17 \\
17\end{array}$ \\
\hline $\mathrm{Ar}$ & $\begin{array}{r}\overline{10,6} \\
8,2\end{array}$ & $\begin{array}{l}0,152 \\
0,159 \\
0,159\end{array}$ & $\begin{array}{l}0,284 \\
0,287 \\
0,287\end{array}$ & $\begin{array}{l}3,80 \\
3,79 \\
3,90\end{array}$ & $\begin{array}{l}0,156 \\
0,158 \\
0,158\end{array}$ & $\begin{array}{l}- \\
-\end{array}$ & $\begin{array}{l}0,162 \\
0,162 \\
0,158\end{array}$ & $\begin{array}{l}3,94 \\
3,90 \\
3,90\end{array}$ & 1,92 & 0,303 & $\begin{array}{l}18 \\
19 \\
19\end{array}$ \\
\hline $\mathrm{Au}$ & 11,0 & 0,210 & 0,385 & 2,86 & 0,212 & 0,212 & 0,214 & 2,91 & 1,44 & 0,306 & 3 \\
\hline $\mathrm{Al}$ & 10,6 & 0,210 & 0,360 & 2,96 & 0,198 & 0,214 & 0,207 & $(2,92)$ & 1,43 & $(0,300)$ & 20 \\
\hline $\mathrm{Pb}$ & $\begin{array}{r}- \\
- \\
8,0 \\
12,0 \\
9,4\end{array}$ & $\begin{array}{l}0,164 \\
0,170 \\
0,174 \\
0,165 \\
0,180\end{array}$ & $\begin{array}{c}- \\
\overline{-} \\
0,321 \\
0,321 \\
0,325\end{array}$ & $\begin{array}{l}- \\
\overline{3}, 40 \\
3,40 \\
3,40\end{array}$ & $\begin{array}{c}- \\
\overline{-} \\
0,176 \\
0,176 \\
0,179\end{array}$ & 0,175 & $\begin{array}{c}- \\
- \\
0,180\end{array}$ & $\begin{array}{c}- \\
- \\
3,49 \\
3,49 \\
3,45\end{array}$ & 1,75 & $\begin{array}{c}\overline{-} \\
\overline{-} \\
0,308 \\
0,308 \\
0,313\end{array}$ & $\begin{array}{r}21 \\
22 \\
3,23 \\
24 \\
11\end{array}$ \\
\hline $\mathrm{Tl}$ & $-8,0$ & $\begin{array}{l}0,164 \\
0,180\end{array}$ & $\begin{array}{l}0,323 \\
0,325\end{array}$ & $\overline{3,30}$ & $\begin{array}{l}0,178 \\
0,179\end{array}$ & 0,179 & $\overline{0,186}$ & $\begin{array}{l}3,46 \\
3,44\end{array}$ & 1,71 & $\begin{array}{l}0,304 \\
0,306\end{array}$ & $\begin{array}{r}21 \\
3,23\end{array}$ \\
\hline $\mathrm{Li}$ & 9,8 & 0,195 & 0,355 & 3,24 & 0,195 & 0,195 & 0,189 & 3,15 & 1,57 & 0,306 & 20 \\
\hline $\mathrm{Na}$ & $\begin{array}{l}- \\
\overline{10,0} \\
9,5\end{array}$ & $\begin{array}{l}0,166 \\
0,152 \\
0,155 \\
0,161\end{array}$ & $\begin{array}{c}\overline{-} \\
\overline{-} \\
0,282 \\
0,290\end{array}$ & $\begin{array}{c}\overline{-} \\
\approx \overline{4,00} \\
3,83\end{array}$ & $\begin{array}{c}\overline{-} \\
\overline{-} \\
0,155 \\
0,160\end{array}$ & 0,159 & $\begin{array}{c}\overline{-} \\
\overline{-} \\
0,160\end{array}$ & $\begin{array}{c}- \\
\overline{-} \\
3,97 \\
3,86\end{array}$ & 1,92 & $\begin{array}{c}- \\
\overline{-} \\
0,298 \\
0,307\end{array}$ & $\begin{array}{l}25 \\
21 \\
26 \\
27\end{array}$ \\
\hline $\mathbf{K}$ & $\begin{array}{l}- \\
\approx 8,0\end{array}$ & $\begin{array}{l}0,129 \\
0,119 \\
0,130\end{array}$ & $\frac{-}{0,234}$ & $\frac{-}{4,64}$ & $\frac{-}{0,129}$ & 0,129 & $\overline{-}$ & $\begin{array}{c}(4,75) \\
\overline{4,78}\end{array}$ & $2,36 / 8$ & $\frac{-}{\overline{0}, 306}$ & $\begin{array}{l}25 \\
21 \\
28\end{array}$ \\
\hline $\mathrm{Rb}$ & - & 0,122 & - & - & - & 0,121 & - & $(5,02)$ & $2,51 / 3$ & $(0,308)$ & 25 \\
\hline $\mathrm{Cs}$ & - & 0,111 & - & - & - & 0,112 & - & $(5,51)$ & $2,70 / 4$ & $(0,302)$ & 25 \\
\hline $\mathrm{Zn}$ & 10,8 & 0,228 & 0,380 & 2,94 & 0,209 & 0,223 & 0,208 & $(2,69)$ & 1,37 & $(0,312)$ & 20 \\
\hline $\mathrm{Cd}$ & 8,3 & 0,203 & 0,366 & 3,06 & 0,201 & 0,201 & 0,200 & 3,06 & 1,52 & 0,306 & 20 \\
\hline $\mathrm{Hg}$ & $\begin{array}{l}- \\
- \\
- \\
- \\
6 \\
\approx 8,0 \\
- \\
8,2 \\
8,3\end{array}$ & $\begin{array}{l}0,174 \\
0,181 \\
0,198 \\
0,171 \\
0,183 \\
0,184 \\
0,185 \\
0,188 \\
0,194 \\
\end{array}$ & $\begin{array}{l}0,342 \\
0,354 \\
0,367 \\
0,354 \\
0,360 \\
0,358 \\
0,365 \\
0,360 \\
0,361 \\
\end{array}$ & $\begin{array}{c}- \\
\overline{-} \\
3,00 \\
3,23 \\
3,00 \\
3,11 \\
- \\
3,10 \\
\approx 3,13\end{array}$ & $\begin{array}{l}0,188 \\
0,195 \\
0,202 \\
0,195 \\
0,198 \\
0,197 \\
0,201 \\
0,198 \\
0,198\end{array}$ & 0,196 & $\begin{array}{c}- \\
\overline{0} \\
0,204 \\
0,190 \\
0,204 \\
0,197 \\
- \\
0,198 \\
0,196\end{array}$ & $\begin{array}{l}3,27 \\
3,16 \\
3,05 \\
3,16 \\
3,11 \\
3,12 \\
3,07 \\
3,11 \\
3,10\end{array}$ & 1,55 & $\begin{array}{l}0,292 \\
0,302 \\
0,313 \\
0,302 \\
0,307 \\
0,306 \\
0,312 \\
0,307 \\
0,308\end{array}$ & $\begin{array}{l}21 \\
29 \\
30 \\
31 \\
32 \\
33 \\
34 \\
35 \\
36\end{array}$ \\
\hline In & $\begin{array}{l}8,5 \\
8,0\end{array}$ & $\begin{array}{l}0,179 \\
0,181\end{array}$ & $\begin{array}{l}0,335 \\
0,340\end{array}$ & $\begin{array}{l}3,30 \\
3,17\end{array}$ & $\begin{array}{l}0,184 \\
0,187\end{array}$ & 0,183 & $\begin{array}{l}0,186 \\
0,193\end{array}$ & $\begin{array}{l}3,34 \\
3,29\end{array}$ & 1,67 & & $\begin{array}{r}20 \\
3,23\end{array}$ \\
\hline $\mathrm{Sn}$ & $\begin{array}{l}- \\
\overline{10,0} \\
10,0\end{array}$ & $\begin{array}{l}0,171 \\
0,177 \\
0,174 \\
0,177\end{array}$ & $\begin{array}{c}- \\
\overline{-} \\
0,335 \\
0,338\end{array}$ & $\begin{array}{l}- \\
\overline{3} \\
3,38 \\
3,20\end{array}$ & $\begin{array}{c}- \\
\overline{-} \\
0,184 \\
0,186\end{array}$ & 0,194 ? & $\begin{array}{c}- \\
\overline{-} \\
0,181 \\
0,191\end{array}$ & $\begin{array}{c}- \\
\overline{-} \\
3,34 \\
3,31\end{array}$ & 1,58 & & $\begin{array}{c}21 \\
37 \\
20 \\
3\end{array}$ \\
\hline $\mathrm{Ga}$ & $\frac{-}{11,0}$ & $\begin{array}{l}0,194 \\
0,192 \\
0,199\end{array}$ & $\begin{array}{l}0,376 \\
0,382 \\
0,388\end{array}$ & $\begin{array}{l}2,80 \\
\overline{2,77}\end{array}$ & $\begin{array}{l}0,207 \\
0,210 \\
0,214\end{array}$ & 0,220 & $\begin{array}{c}0,219 \\
- \\
0,221\end{array}$ & $\begin{array}{l}2,98 \\
2,93 \\
2,88\end{array}$ & 1,39 & & $\begin{array}{r}31 \\
21 \\
3\end{array}$ \\
\hline $\mathrm{Ge}$ & 8,0 & 0,198 & 0,391 & 2,70 & 0,215 & 0,220 & 0,227 & 2,86 & 1,39 & & 3 \\
\hline $\mathrm{Sb}$ & 6,1 & 0,173 & 0,340 & 3,12 & 0,187 & 0,190 & 0,196 & 3,29 & 1,61 & & 38,39 \\
\hline $\mathrm{Bi}$ & $\begin{array}{r}- \\
7-8 \\
8,0 \\
8,0 \\
7,7\end{array}$ & $\begin{array}{l}0,170 \\
0,166 \\
0,164 \\
0,160 \\
0,175\end{array}$ & $\begin{array}{c}-\overline{327} \\
0,327 \\
0,335 \\
0,336 \\
0,330\end{array}$ & $\begin{array}{l}-\overline{3,32} \\
3,20 \\
3,35\end{array}$ & $\begin{array}{c}-\overline{0}, 180 \\
0,184 \\
0,185 \\
0,182\end{array}$ & 0,168 & $\begin{array}{c}\overline{-} \\
0,184 \\
0,191 \\
0,183\end{array}$ & $\begin{array}{l}- \\
3,42 \\
3,34 \\
3,33 \\
3,39\end{array}$ & 1,82 & & $\begin{array}{r}22 \\
3 \\
24 \\
24 \\
11\end{array}$ \\
\hline
\end{tabular}

- $(\ldots)=$ aus $\left(\sin \vartheta_{1} / \lambda\right)$ exp. berechnet.

Fußnoten auf der nächsten Seite

Tab. 1. Experimentelle und berechnete Werte von $\sin \vartheta / \lambda$ und kürzeste Atomabstände $r_{1}$. 
- Die Tab. 1 gliedert sich in einen oberen und einen unteren Teil. Die aus dem II. Maximum berechneten $\sin \vartheta_{\mathrm{I}} / \lambda$-Werte (Spalte 6$)$ im oberen Teil stimmen mit den experimentellen $\left[\sin \vartheta_{1} / \lambda\right)_{\text {exp. }}$-Werte] bis auf $\mathrm{Al}$ und $\mathrm{Zn}$ recht gut überein. Bei $\mathrm{Al}$ und $\mathrm{Zn}$ liegt offenkundig eine Oxydbildung vor; hierfür spricht das kleine Maximum bei $\sin \vartheta / \lambda \approx 0,1$ bzw. 0,115 (vgl. Abb. 12). Die Oxydbildung scheint das II. Maximum der Kugelmodell-Anordnung nach kleinen Werten von $\sin \vartheta / \lambda$ zu verlagern. Im unteren Teil der Tab. 1 sind die experimentellen $\sin \vartheta_{1} / \lambda$. Werte z. Tl. beträchtlich kleiner als die aus dem II. Maximum berechneten $\sin \vartheta_{\mathrm{I}} / \lambda$-Werte, d. h. das I. Flüssigkeits-Maximum ist nach der Seite der kleinen $\sin \vartheta / \lambda$-Werte verschoben. Dieser Befund ist kein Zufall, er ist sicherlich strukturell bedingt; er deutet an, daß die Lage des 1. Maximums durch verschiedene Beugungsvorgänge hervorgerufen wird. Die aus dem II. Flüssigkeits-Maximum errechneten kürzesten Atomabstände $\left(r_{1}\right)_{\text {ber. bzw. Atomradien }}$ $\left(a_{\mathrm{K}}{ }^{*}=\frac{1}{2} r_{1}\right)$ stimmen nach Tab. 1 , Spalte 9 mit den

Fußnoten zu Tabelle 1:

16 J. Reekie u. T. S. Hutchison, Phys. Rev. 91, 490 [1953] 92, 827 [1953]. - J. Reekie, T. S. Hutchison u. C. F. A. Beaumont, Proc. Phys. Soc., Lond. A 66, 409 [1953]. C. F. A. Beaumont u. J. Reekie, Proc. Roy. Soc., Lond. A 228, 363 [1955].

17 W. L. Gordon, C. H. Shaw u. I. G. Daunt, Phys. Rev. 96, 1444 [1956]. - D. G. Hurst u. D. G. Henshaw, Phys. Rev. 91, 1222 [1953]; 100, 994 [1955].

18 K. Lark-Horovitz u. E. P. Miller, Nature, Lond. 146, 459 [1940].

19 A. Eisenstein u. N. S. Gingrich, Bull. Amer. Phys. Soc. 15, Nr. 2, April [1940] ; Phys. Rev. 58, 307 [1940] ; 62, 261 [1942]. - D. G. Henshaw, D. G. Hurst u. N. K. Pope, Phys. Rev. 92, 1229 [1953].

20 G. Gamertsfelder, Phys. Rev. 55, 1116 [1939]; 57, 1055 [1940] ; 59, 926 [1941]. - J. Chem. Phys. 9, 450 [1941].

21 F. Sauerwald u. W. Teske, Z. anorg. u. allg. Chem. 210, 247 [1933]. - F. Sauerwald u. E. Osswald, Z. anorg. u. allg. Chem. 257, 195 [1948].

22 J. T. Randall u. H. P. Rooksby, Trans. Faraday Soc. 33, 109 [1937]. - J. T. Randall, The Diffraction of X-Rays and Electrons by Amorphous Solids, Liquids and Gases, Chapman \& Hall, Ltd., London 1934.

23 R. Glocker u. H. Hendus, Ann. Phys., Lpz. 43, 513 [1943].

24 O. Chamberlain, Phys. Rev. 77, 305 [1950].

25 J. T. Randall u. H. P. Rooksby, Nature, Lond. 130, 473 [1932].

26 L. P. Tarasov u. B. E. Warren, J. Chem. Phys. 4, 236 [1936].

27 F. H. Trimble u. N.S. Gingrich, Phys. Rev. 53, 278 [1938].

28 C. D. Thomas u. N. S. Gingrich, J. Chem. Phys. 6, 411 u. 659 [1938]. - N. S. Gingrich, Rev. Mod. Phys. 15, 90 [1943].

29 J. A. Prins, Z. Phys. 56, 617 [1929].

30 P. Debye u. H. Menke, Phys. Z. 31, 797 [1930]; Ergebn. d. techn. Röntgenkunde II, 1 [1931].

31 H. Menke, Phys. Z. 33, 593 [1932]. aus der Literatur bekannten Werten $a_{12}{ }^{*}$ für ZwölferKoordination von GoLDschmidt im allgemeinen recht gut überein, besonders die im oberen Teil der Tab. 1 .

\section{b) Berechnung von $\sin \vartheta_{\mathrm{I}} / \lambda$ bei bekanntem Atomradius}

Randall und Rooksby ${ }^{25}$ suchen das 1. Maximum in den Streukurven von flüssigem $\mathrm{Na}, \mathrm{K}, \mathrm{Rb}$ und Cs als einen Reflexionsvorgang an der (110)-Ebene des zugehörigen Gitters zu deuten. Die dabei erreichte Übereinstimmung ist recht unbefriedigend. Weiter weist Hendus ${ }^{3}$ bei flüssigem $\mathrm{Au}, \mathrm{Pb}$, In und $\mathrm{Tl}$ auf die gute Übereinstimmung ${ }^{40} \mathrm{der}$ Interferenzlagen für das 1. Maximum mit der der dichtest belegten Ebene des jeweiligen Gitters [(111)-Ebene] hin; bei $\mathrm{Tl}$ ist eine solche Übereinstimmung ebenfalls für die dichtest belegte Ebene des hexagonalen Gitters [(001)-Ebene] vorhanden. Schließlich will Hosemann $^{8}$ das 1. Maximum der Intensitätskurve von $\mathrm{Au}$ wegen seiner überragenden Höhe als einen „eigentlichen Reflex" gedeutet wissen, auch die

32 H. Hendus, Z. Naturforschg. 3 a, 416 [1948].

33 H. Richter u. D. Handtmane, Z. Metallkde., 49, [1958].

34 R. E. Jennings (vgl. Acta Cryst. 5, 1 [1952], Fig. 3).

35 R. E. Smallman u. B. R. T. Frost, Acta Met. 4, 611 [1956].

36 G. H. Vineyard, J. Chem. Phys. 22, 1665 [1954].

37 J. A. Prins, Trans. Faraday Soc. 33, 110 [1937].

38 H. Hendus u. H. K. F. Müller, Z. Naturforschg. 10 a, 254 [1955].

39 H. Hendus u. H. K. F. Müller, Z. Naturforschg. 12 a, 102 [1957].

40 Dieser Befund läßt sich leicht verifizieren. Aus dem flächenzentriert-kubischen bzw. hexagonalen Gitter folgt nämlich mit $c_{\mathrm{H}}=2 \sqrt{2 / 3} a_{\mathrm{H}}$

$$
\begin{aligned}
\left(\frac{\sin \vartheta}{\lambda}\right)_{(111)}^{\text {kub. }} & =\frac{1}{2 d_{(111)}}=\sqrt{\frac{3}{4} \frac{1}{a_{\mathrm{K}}}} \\
& \text { bzw. }\left(\frac{\sin \vartheta}{\lambda}\right)_{(001) 2}^{\text {hex. }}=\frac{1}{2 d_{(001) 2}} \sqrt{\frac{1}{c_{\mathrm{H}}^{2}}}=\sqrt{\frac{3}{8} \frac{1}{a_{\mathrm{H}}}}
\end{aligned}
$$

oder mit $a_{\mathrm{H}}=r_{1}=a_{\mathrm{K}} / 2 \cdot \sqrt{2}$

$$
\begin{aligned}
& \left(\frac{\sin \vartheta}{\lambda}\right)_{(111)}^{\text {kub. }}=\left(\frac{\sin \vartheta}{\lambda}\right)_{(001) 2}^{\text {hex. }}=\sqrt{\frac{3}{8} \frac{1}{a_{\mathrm{H}}}} \\
& =\sqrt{\frac{3}{8}} \frac{1}{r_{1}}=0,6125 \cdot \frac{1}{r_{1}} \approx \frac{\sin \vartheta_{\mathrm{I}}}{\lambda}=0,6147 \cdot \frac{1}{r_{1}},
\end{aligned}
$$

d. h. das I. Flüssigkeits-Maximum des statistischen Kugelmodells und die Raumgitter-Interferenzen von (111) kub. und $(002)_{\text {hex. }}$ treten praktisch unter demselben Winkel auf. Den gleichen Zahlenfaktor 0,6125 liefert auch die (110)-Ebene des raumzentriert-kubischen Gitters

$$
r_{1}{ }^{\prime}=a_{\mathrm{K}^{\prime}} / 2 \cdot \sqrt{3} \text {; }
$$

hier ist

$$
\frac{\sin \vartheta_{(110)}}{\lambda}=\sqrt{\frac{3}{8}} \frac{1}{r_{1}{ }^{\prime}}=0,6125 \cdot \frac{1}{r_{1}{ }^{\prime}} .
$$


Schärfe dieses Maximums spricht hierfür. Man sieht, daß es nicht an Versuchen fehlt, das Streubild von Metallschmelzen als einen Reflexionsvorgang an Netzebenen zu deuten.

Macht man andererseits die Annahme, daß in einatomigen Metallschmelzen die Atome nach Art des statistischen Kugelmodells angeordnet sind, so kann man nach oben für die Lage des I. FlüssigkeitsMaximums schreiben ${ }^{41}$ (vgl. auch Anm. ${ }^{40}$ )

$$
\sin \vartheta_{\mathrm{I}} / \lambda=0,6147 \cdot 1 / r_{1}=0,307 \cdot 1 / a_{\mathrm{K}}{ }^{*} .
$$

Diese Gleichung gilt für jede Koordination, d. h. das Produkt aus der Winkellage $\sin \vartheta_{\mathrm{I}} / \lambda$ und dem zugehörigen Atomradius $a_{\mathrm{K}}{ }^{*}$ besitzt immer den konstanten Wert 0,307. Multipliziert man die aus der Lage des II. Maximums erhaltenen $\left(\sin \vartheta_{\mathrm{I}} / \lambda\right)_{\text {ber.- }}$ Werte der Tab. 1 (Spalte 6) mit den in der vorletzten Spalte angegebenen Atomradien $a_{12}{ }^{*}$ für ZwölferKoordination, so gelangt man zu den Zahlenwerten der letzten Spalte. Sieht man von $\mathrm{Al}, \mathrm{Zn}$ und z. Tl. von $\mathrm{Hg}$ [ältere $(\sin \vartheta / \lambda)$-Werte] ab, so ergibt sich in guter Übereinstimmung mit der Berechnung als mittlerer Wert für die Konstante 0,306; auch die Alkali-Metalle ${ }^{42}$ ergeben denselben Wert. Dieser Befund ist ein unmittelbarer Beweis dafür, daß die Multiplikation mit den richtigen (zugehörigen) Atomradien-Werten erfolgte. Die Multiplikation mit den Atomradien $a_{8}{ }^{*}$ für Achter-Koordination liefert dagegen im Mittel den zu kleinen Wert 0,296. Trotz des geringen Unterschiedes der Atomradien $a_{\mathrm{K}}{ }^{*}$ für Zwölfer- und Achter-Koordination spricht das hier erhaltene Ergebnis eindeutig für das Vorliegen der dichtesten Atompackung (Zwölfer-Koordination) in einatomigen Metallschmelzen.

Die Lagen der Flüssigkeits-Maxima $\left[\sin \vartheta_{\mathrm{I}, \mathrm{II}} / \hat{\lambda}\right]$ kann man also berechnen, und zwar für die I. Koordination aus dem Atomradius $a_{\mathrm{K}}{ }^{*}$. Für die weitere Berechnung und zur Konstruktion der Abb. 7 wurde die Konstante zu 0,306 gewählt, wie sie sich aus dem oberen Teil der Tab. 1 als experimentell gefundener Mittelwert ergibt. Nach der obigen Beziehung wurden für den Fall der dichtesten Atompackung die in Tab. 1, Spalte 7, zusammengestellten Zahlenwerte als Lagen für das I. Flüssigkeits-Maximum der bisher untersuchten Metallschmelzen erhalten.

41 Vorausgesetzt, daß die Lagen der Maxima in der Intensitätskurve allein von einem (kürzesten) Atomabstand bestimmt werden. - Das I. Maximum der Kugelmodell-Anordnung für $r_{2}=r_{1} \sqrt{2}$ müßte bei $\sin \vartheta_{\mathrm{T}} / \lambda=0,651 / r_{2}$ auftreten.
Die aus den Atomradien der Zwölfer-Koordination ermittelten Werte von $\left(\sin \vartheta_{\mathrm{I}} / \lambda\right)_{\text {ber. }}$ (vgl. Tab. 1, Spalte 7) zeigen im oberen Teil der Tab. 1 eine recht gute Übereinstimmung mit den experimentellen (vgl. Spalte 3) und den aus dem II. Maximum berechneten $\sin \vartheta_{\mathrm{I}} / \lambda$-Werten (vgl. Spalte 6). Diese Übereinstimmung ist bei den Alkali-Metallen besonders gut; bei $\mathrm{Al}$ und $\mathrm{Zn}$ besteht Übereinstimmung nur mit den experimentellen Werten von $\sin \vartheta_{1} / \lambda$, d. h. die Lage des I. Flüssigkeits-Maximums wird durch die Oxydbildung nicht wesentlich beeinflußt. So findet man für $\mathrm{Zn}$ mit $\left(\sin \vartheta_{1} / \lambda\right)_{\text {exp. }}=0,228$ nach $\sin \vartheta_{\mathrm{I}} / \lambda=0,612 \cdot 1 / r_{1}$ als Atomradius $a_{\mathrm{ber}}^{*}$ $=1,35 \AA$, einen Wert, der mit dem bekannten Atomradius $a_{12}{ }^{*}=1,37 \AA$ im besten Einklang steht. Der aus dem 2. Maximum $\left(\sin \vartheta_{2} / \lambda=0,38\right)$ nach $\sin \vartheta_{\text {II }} / \lambda=1,119 \cdot 1 / r_{1}$ berechnete Atomradius $a_{\text {ber. }}^{*}$ $=1,47 \AA$ stimmt dagegen weniger gut; auffallenderweise stimmt er aber mit dem Ergebnis der FourierAnalyse von Gamertsfelder ${ }^{20}\left(r_{1}=2,94 \AA\right)$ überein. Im unteren Teil der Tab. 1 besteht für die aus dem Atomradius $a_{12}{ }^{*}$ berechneten Werte von $\sin \vartheta_{\mathrm{I}} / \lambda_{\text {. }}$ (vgl. Spalte 7) mit den experimentellen Werten von $\sin \vartheta_{1} / \lambda$ (vgl. Spalte 3 ) keine, dagegen mit den aus dem II. Flüssigkeits-Maximum ermittelten Werten in Spalte 6 zum großen Teil eine recht gute Übereinstimmung, weniger gut ist diese ${ }^{43}$ bei $\mathrm{Bi}$. Hier scheint sich in den Werten von $\left(\sin \vartheta_{1} / \lambda\right)_{\text {exp. }}$ die Schmelz-Anomalie von $\mathrm{Bi}$ widerzuspiegeln.

\section{c) Berechnung von $\sin \vartheta_{\mathrm{I}} / \lambda$ aus dem Ergebnis der Fourier-Analyse}

Ist der Atomabstand $r_{1}$ durch Fourier-Analyse der Streukurve bekannt, so kann man die $\sin \vartheta_{v} / \lambda$-Werte für die Flüssigkeits-Maxima unmittelbar bestimmen. In Tab. 1, Spalte 8 sind die Lagen für das I. Maximum aufgeführt, wie man sie nach der Gleichung $\sin \vartheta / \lambda=0,612 \cdot 1 / r_{1}$ errechnet. Wird der Verlauf der Streukurve praktisch nur durch das statistische Kugelmodell festgelegt, dann sind eventuelle Abweichungen in den $\sin \vartheta_{v} / \lambda$-Werten zwischen Experiment und Berechnung sicherlich als Fehler im Gang der Analyse zu deuten. Sind dagegen verschiedene Beugungsvorgänge am Zustandekommen der Intensitätskurve,

42 Für die Elemente der unteren Gruppe in Tab. 1 besteht eine solche Übereinstimmung nicht.

43 Abweichungen zwischen Berechnung nach a) bzw. b) und Experiment sind als Beeinflussung der Interferenzlagen des statistischen Kugelmodells durch andere Beugungsvorgänge zu deuten. 


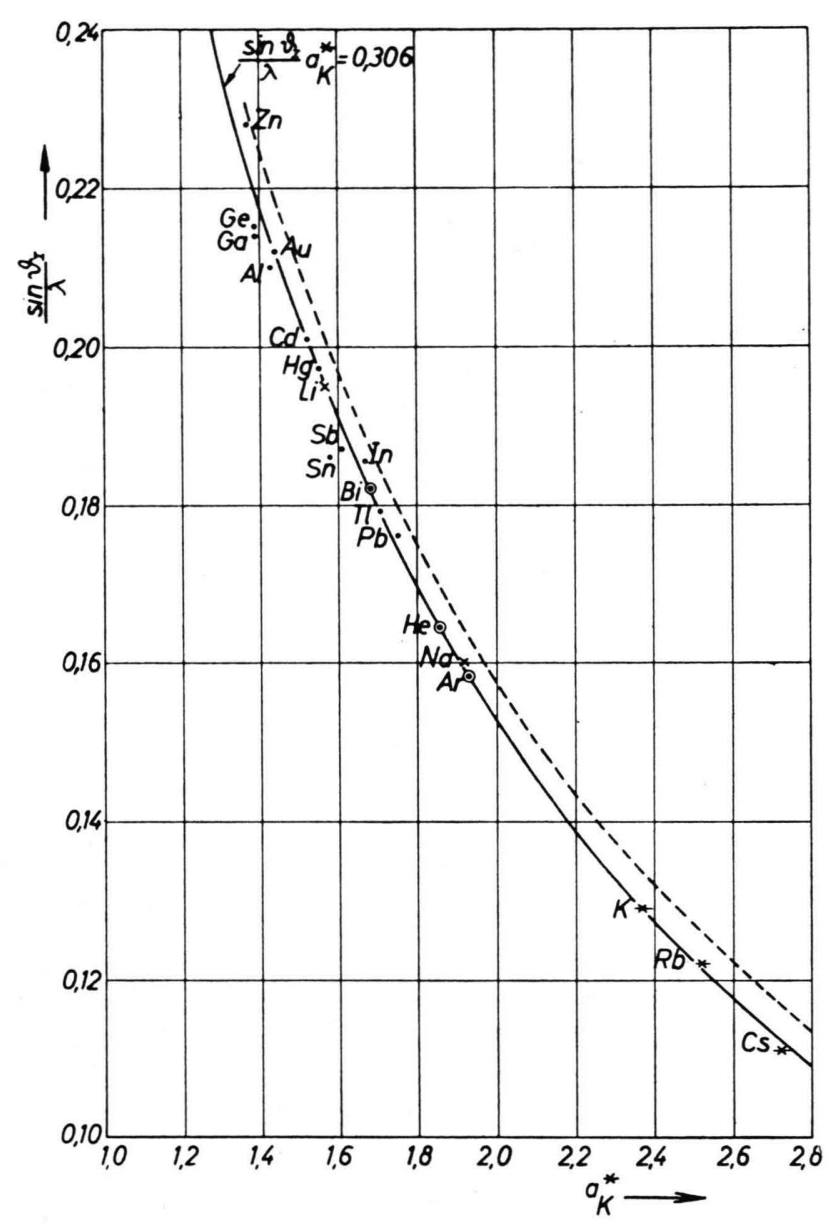

Abb. 7. Lage des I. Flüssigkeits-Maximums geschmolzener Metalle als Funktion des Atomradius.

insbesondere an der Fixierung des 1. Maximums beteiligt, dann ist, wie der untere Teil der Tab. 1 zeigt (vgl. Spalte 8 mit 3 bzw. 6), kaum eine Übereinstimmung zwischen den experimentellen und berechneten $\sin \vartheta_{v} / \lambda$-Werten, zumindest nicht für das 1. Maximum, vorhanden. Hierbei ist $\mathrm{zu}$ beachten, daß die Berechnung nur die Lagen der Maxima der Interferenz-Funktion $\sin k s r_{1} / k s r_{1}$, die Analyse dagegen den gesamten Intensitätsverlauf berücksichtigt.

44 Es wurden also die $\left(\sin \vartheta_{\mathrm{I}} / \lambda\right)$ ber.-Werte über den richtigen (zugehörigen) Atomradien-Werten aufgetragen.

45 Dieser Befund steht mit den bisherigen Ergebnissen der Fourier-Analyse der Streukurven von Alkali-Schmelzen keineswegs in Widerspruch; denn GAMERTSFELDER ${ }^{20}$ gibt für Li als Atomzahl $N_{1}=9,8$ an und für $\mathrm{Na}$ ermittelt man aus den Atomverteilungskurven von $\mathrm{T}_{\mathrm{ARASO}}$ und $\mathrm{W}_{\mathrm{AR}}$ REN ${ }^{26}$ sowie Trimble und Gingrich ${ }^{27}$ als Atomzahl $N_{1}=10,0$ d) Berechnete und experimentelle Werte von $\sin \vartheta_{\mathrm{I}} / \lambda$ als Funktion des Atomradius

Einen unmittelbaren Einblick in den Aufbau der einatomigen Metallschmelzen vermittelt Abb. 7. Hier stellt die ausgezogene Kurve die $\sin \vartheta_{\mathrm{I}} / \lambda$-Werte als Funktion des Atomradius $a_{\mathrm{K}}{ }^{*}$ dar, und zwar gemä $\beta$ der Gleichung $\sin \vartheta_{\mathrm{I}} / \lambda \cdot a_{\mathrm{K}}{ }^{*}=0,306$. Wie oben bereits bemerkt, gilt diese Beziehung bzw. die untere Kurve in Abb. 7 (gleichseitige Hyperbel) für jede Koordination, d. h. die $\sin \vartheta_{\mathrm{I}} / \lambda$-Werte der Koordination $\mathrm{K}$, über den zugehörigen Atomradien $a_{\mathrm{K}}{ }^{*}$ aufgetragen, liegen auf der ausgezogenen Kurve, und zwar liegen sie bei einem um so kleineren Werte von $\sin \vartheta_{\mathrm{I}} / \lambda$, je höher die Koordinationszahl bzw. je größer der Atomradius $a_{\mathrm{K}}{ }^{*}$ ist. Sucht man in Abb. 7 (untere Kurve) für die aus dem II. Maximum der Intensitätskurve berechneten $\sin \vartheta_{\mathrm{I}} / \lambda$-Werte der Tab. 1 (vgl. Spalte 6) die zugehörigen AtomradienWerte auf der Abszissenachse auf, so sieht man, daß diese Werte mit den Atomradien $a_{12}{ }^{*}$ für ZwölferKoordination praktisch zusammenfallen. Um die Streuungen der berechneten $\sin \vartheta_{\mathrm{I}} / \lambda$-Werte um die $\sin \vartheta_{\mathrm{I}} / \lambda \cdot a_{12}{ }^{*}$-Kurve deutlich hervortreten zu lassen, wurden die $\left(\sin \vartheta_{\mathrm{I}} / \lambda\right)_{\text {ber. }}$-Werte (vgl. Tab. 1, Spalte 6) über den Atomradien $a_{12}{ }^{*}$ für Zwölfer-Koordination in Abb. 7 aufgezeichnet. Man stellt fest, daß die aus dem II. Maximum berechneten $\sin \vartheta_{\mathrm{I}} / \lambda$-Werte für die untersuchten Metallschmelzen - von einer Korrektion auf Zimmertemperatur wurde Abstand genommen - ausnahmslos auf der unteren Kurve ${ }^{44}$ liegen; das gilt besonders für die Alkali-Metalle, d. h. selbst in diesen Schmelzen sind die Atome, abweichend vom zugehörigen Gitter mit Achter-Koordination, dichtest ${ }^{45}$ gepackt. Bei Vorliegen einer AchterKoordination müßte man einen $\sin \vartheta_{\mathrm{I}} / \lambda$-Wert erhalten (vgl. Tab. 8), der auf die gestrichelte Kurve ${ }^{46}$ fällt, wenn man ihn beim Atomradius $a_{12}{ }^{*}$ für Zwölfer-Koordination aufträgt. Trägt man diesen Wert dagegen bei dem zugehörigen Atomradius $a_{8}{ }^{*}$ für Achter-Koordination auf, dann muß er gemäß der Beziehung $\left(\sin \vartheta_{\mathrm{I}} / \lambda\right)_{8} a_{8}{ }^{*}=0,306$ wieder auf die ausgezogene Kurve der Abb. 7 fallen. Weiter sieht man, da $ß$ nicht ein einziger $\sin \vartheta_{\mathrm{I}} / \lambda$-Wert der bisher

bzw. 9,5. Für $\mathrm{K}$ geben Thomas und Gingrich ${ }^{28}$ allerdings $N_{1} \approx 8,0$ an.

${ }_{46}$ Die gestrichelte Kurve (transformierte Achter-Koordinationskurve) entsteht aus der ausgezogenen durch die Koordinaten-Transformation

$$
\begin{aligned}
& x^{\prime}=a_{8}^{*}+\left(a_{12}^{*}-a_{8}^{*}\right)=a_{12}{ }^{*}, \\
& y^{\prime}=y_{8}=\left(\sin \vartheta_{\mathrm{I}} / \lambda\right)_{8} .
\end{aligned}
$$


untersuchten Schmelzen von einatomigen Metallen die transformierte Achter-Koordinationskurve erreicht, geschweige denn auf ihr zu liegen kommt. Obwohl der Unterschied in den $\sin \vartheta_{\mathrm{I}} / \lambda$-Werten für Achter- und Zwölfer-Koordination nur etwa 3 bis $4 \%$ beträgt, darf man aus der Streuung der Meßpunkte um die ausgezogene Kurve der Abb. 7 wie oben bei der Produkt-Bildung (vgl. S. 905) schließen, $\mathrm{da}$ eine Atomgruppierung mit Achter-Koordination in den Metallschmelzen offensichtlich nicht anzutreffen ist. Alle Metalle streben in ihren Schmelzen dem Zustand der dichtesten Atompackung zu.

Bei $\mathrm{Ga}, \mathrm{Ge}, \mathrm{Sn}$ und $\mathrm{Sb}$ sind kleine Abweichungen vom Kurvenverlauf von etwa $3 \%$ vorhanden, geringer sind die Abweichungen bei In und $\mathrm{Pb}^{47}$, aber in umgekehrtem Sinne. Vermutlich ist hier sogar die Lage des II. Flüssigkeits-Maximums der Intensitätskurve durch andere Beugungsvorgänge leicht gestört, oder aber die Kenntnis der Atomradien-Werte ist nicht ausreichend. Bei $\mathrm{Al}$ und $\mathrm{Zn}$ wurden die experimentellen $\sin \vartheta_{1} / \lambda$-Werte, bei In der aus dem II. Maximum (vgl. Gamertsfelder ${ }^{20}$ und Hendus ${ }^{3}$ ) errechnete mittlere Wert von $\sin \vartheta_{\mathrm{I}} / \lambda$ und bei $\mathrm{Hg}$ der Wert von Richter und Handtmanv ${ }^{33}$ eingezeichnet. Als Atomradius für Ar gibt WinkLer ${ }^{48}$ (S. 168) an: $a_{12}{ }^{*}=1,92 \AA$; aus Abb. 7 ermittelt man $a^{*}$ $=1,93 \AA$ und für $\mathrm{He}: a^{*}=1,87 \AA$. Allein bei $\mathrm{Bi}$ mußte, sicherlich wegen der Schmelz-Anomalie, der Atomradius für die Zwölfer-Koordination $a_{1}{ }^{*}=1.82 \AA$ auf $a_{\text {her }}=1.68 \AA$ erniedrigt werden, damit der aus dem II. Flüssigkeits-Maximum berechnete Mittelwert $\sin \vartheta_{\mathrm{T}} / \lambda=0,182$ (vgl. Tab. 1), der sonst weit über die gestrichelte Kurve der Abb. 7 hinausfiele, auf der ausgezogenen Kurve zu liegen kommt. Der $\left(\sin \vartheta_{\mathrm{T}} / \lambda\right)_{\mathrm{v}}$-Wert für geschmolzenes $\mathrm{Bi}$ muß auf die Kurve fallen, solange die InterferenzLagen in der Streukurve allein von einem (kürzesten) Atomabstand festgelegt werden. Der mittlere experimentelle $\sin \vartheta_{1} / \lambda$-Wert für Bi [ $\left(\sin \vartheta_{1} / \lambda_{\mathrm{M}}=0,168\right]$ ist erheblich geringer als der aus dem II. Maximum errechnete Wert von $\left(\sin \vartheta_{\mathrm{T}} / \lambda_{\mathrm{Y}}=0.182\right.$. Bei dem gegebenen Atomradius von Bi für Zwölfer-Koordinationen $a_{12}{ }^{*}=1,82 \AA$ fällt dieser mittlere $\sin \vartheta_{1} / \lambda_{\text {-Wert }}$ auf die ausgezogene Kurve in Abb. 7, doch ist er nach den späteren Ausführungen bei Bi auszuschlieBen.

Die vorangegangenen Betrachtungen gestatten also, aus den Interferenz-Lagen der Intensitätskur-

47 Bezüglich der $\left(\sin \vartheta_{\mathrm{T}} / \lambda\right)_{\text {ber. }}$ - Werte für $\mathrm{Pb}$ und Bi beachte Anm. ${ }^{15}$. ven auf eine dichteste Packung der Atome in einatomigen Metallschmelzen zu schließen. Weiter kann man aus der Lage des II. bzw. III. FlüssigkeitsMaximums den kürzesten Atomabstand $r_{1}$ sowie die Lagen der Flüssigkeits-Maxima berechnen. Bestehen zwischen den experimentellen und berechneten Werten von $\left(\sin \vartheta_{1} / \lambda\right)_{\text {exp. }}$ und $\left(\sin \vartheta_{\mathrm{I}} / \lambda\right)_{\text {ber. }}$ starke $\mathrm{Ab}$ weichungen (vgl. unteren Teil der Tab. 1), so sind Flächengitter-, evtl. Raumgitter-Interferenzen von Schichtpaketen mit im Spiele, bestehen dagegen keine wesentlichen Unterschiede (vgl. oberen Teil der Tab. 1), so kann selbst in diesem Falle die I. Flüssigkeits-Interferenz der dichtesten Atompackung (statistisches Kugelmodell) auch durch Beugungsvorgänge an Schichtpaketen geringfügig verlagert sein.

\section{Struktur geschmolzener einatomiger Metalle}

Vergleicht man die Streukurven von flüssigem $\mathrm{Au}$, $\mathrm{Pb}, \mathrm{Li}, \mathrm{Na}, \mathrm{Tl}, \mathrm{Cd}$ u. a. (obere Gruppe der Tab. 1) miteinander, so fällt auf, daß hier das 1. Maximum einen ungestörten, fast symmetrischen Verlauf besitzt, ganz im Gegensatz zu dem der Streukurven von geschmolzenem $\mathrm{Bi}, \mathrm{Sb}, \mathrm{Ge}, \mathrm{Ga}$ u. a. (untere Gruppe der Tab. 1), wo der obige ungestörte Verlauf offensichtlich von einem weiteren Beugungsvorgang überlagert ist. Weiter ist der bei $\mathrm{Bi}, \mathrm{Sn}$ u. a. zu beobachtende steile Abfall des 1. Maximums der Streukurve nach der Seite der kleinen $\sin \vartheta / \lambda$. Werte bemerkenswert. Das unterschiedliche Verhalten der beiden Gruppen spiegelt sich auch in Tab. 1 wieder. Hier sind im oberen Teil die Unterschiede in den experimentellen $\sin \vartheta_{1} / \lambda$-Werten und den aus dem II. Maximum berechneten Werten von $\sin \vartheta_{\mathrm{T}} / \lambda$ z. Tl. sehr gering, aber um so größer sind sie im unteren Teil. Die Schichtpaket-Bildung in einatomigen Metallschmelzen ist es, die Form und Lage speziell des 1. Flüssigkeits-Maximums beeinflußt. Der Streubeitrag der Schichtnakete auf den Verlauf der Intensitätskurve einiger Metallschmelzen soll jetzt diskutiert und deren Aufbau eingehend betrachtet werden. Dabei werden von den beiden Gruppen jeweils ein bzw. zwei Beispiele ausführlich behandelt.

\section{a) Wismut}

Zur Diskussion der in geschmolzenem Bi (Schmelzpunkt $=271^{\circ} \mathrm{C}$ ) vorliegenden Verhältnisse sind in

48 H. G. F. Winrler, Struktur u. Eigenschaften der Kristalle, Springer-Verlag, Berlin 1955. 


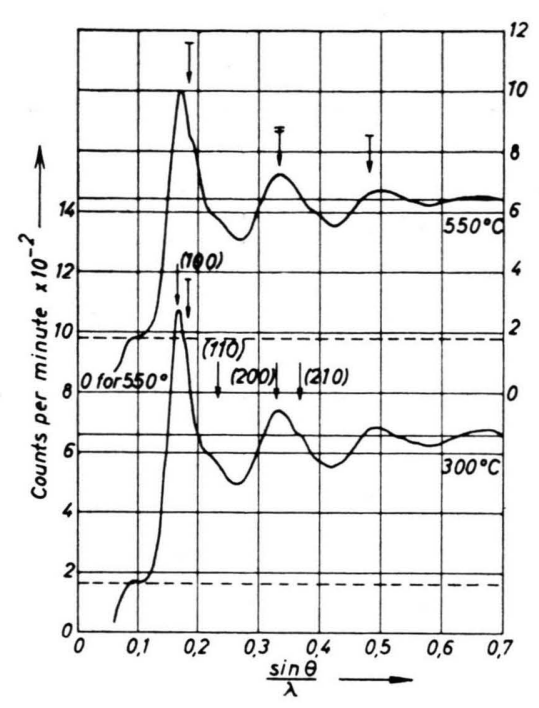

Abb. 8 a. Intensitätskurven von geschmolzenem $\mathrm{Bi}$ bei 300 und $550^{\circ} \mathrm{C}$ (Neutronen-Beugungsaufnahme).

$\downarrow=$ berechnete Lagen der Flüssigkeits-Maxima,

$\downarrow=$ berechnete Lagen der Flächengitter-Interferenzen (100), (110) und (200).

Abb. 8 a zwei Streukurven einer Bi-Schmelze bei verschiedenen Temperaturen wiedergegeben. Diese Kurven wurden von Sharrah und Smith ${ }^{11}$ durch Neutronen-Beugung mit $\lambda=1,16 \AA$ erhalten. Es sei zunächst die $300{ }^{\circ} \mathrm{C}$-Kurve betrachtet. Berechnet man nach oben aus der Lage des II. Maximums den kürzesten. Atomabstand $r_{1}$ und mit ihm $\left(r_{1}=3,39 \AA\right)$ die Lage des I. Flüssigkeits-Maximums ${ }^{49}$, so kommt das I. Maximum mit $\sin \vartheta_{\mathrm{I}} / \lambda=0,182$, in Abb. $8 \mathrm{a}$ durch den Pfeil $\bar{\downarrow}$ hervorgehoben, gerade auf der leichten Wellung im abfallenden Verlaufe des 1. Ma-

49 Oder kürzer $\left(\sin \vartheta_{\mathrm{I}} / \lambda\right)_{\text {ber. }}=0,55\left(\sin \vartheta_{2} / \lambda\right)$ exp. .

50 Die Streu-Intensität für ein Haufwerk durchsichtiger Flächengitter ist nach v. LAUE ${ }^{51}$ darstellbar durch (vgl. auch $\mathrm{W}_{\text {ARREN }}{ }^{52}$ )

$$
I_{(h k 0)}=C \frac{1}{\sin \vartheta \sqrt{\sin ^{2} \vartheta-\sin ^{2} \vartheta_{(h k 0)}}}
$$

mit $C=$ const , $\vartheta=$ Winkel zwischen der Streurichtung $\mathfrak{s}$ und der „mittleren“ Richtung $\mathfrak{s}_{0}$ der einfallenden Strahlung und $\vartheta(h k 0)=$ Winkellage der betrachteten $(h k 0)$ Flächengitter-Interferenz. Der Streuwinkel $\vartheta_{(h k 0)}$ errechnet sich nach der BragGschen Gleichung zu $\sin \vartheta(h k 0) / \lambda$ $=1 / 2 d_{(h k 0)}$. Bei kubischen Gittern mit der (001)-Ebene als Basisfläche ist

$$
\begin{aligned}
& d_{(100)}=\frac{a_{\mathrm{K}}}{\sqrt{1}}, \quad d_{(110)}=\frac{a_{\mathrm{K}}}{\sqrt{2}}, d_{(200)}=\frac{a_{\mathrm{K}}}{\sqrt{4}} ; \\
& d_{(210)}=\frac{a_{\mathrm{K}}}{\sqrt{5}} ; d_{(220)}=\frac{a_{\mathrm{K}}}{\sqrt{8}} \text { usf. }
\end{aligned}
$$

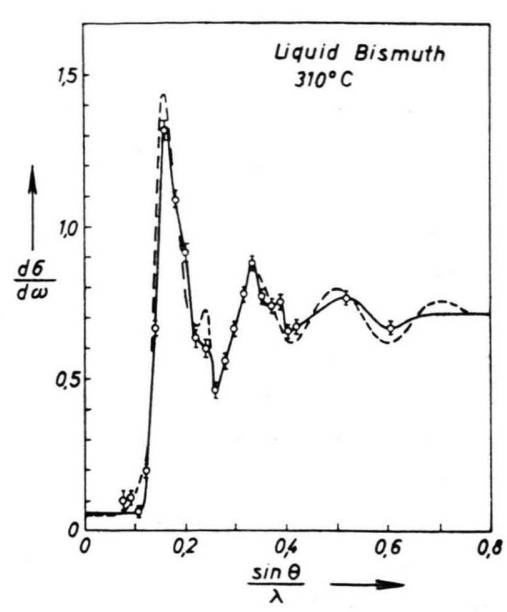

Abb. 8 b. Intensitätskurven von geschmolzenem Bi bei $310^{\circ} \mathrm{C}$ (Neutronen-Beugungsaufnahme).

ximums zu liegen. Die Lagen für die Maxima der Kugelmodell-Struktur aus der 300 - und $550^{\circ} \mathrm{C}$-Kurve von Sharrah und Smith ${ }^{11}$ sind mit denen von Chamberlain ${ }^{24}$ in den ersten drei Spalten der Tab. 2 zusammengestellt, ebenso die hierfür berechneten Werte. Dabei geben die untereinanderstehenden Zahlenwerte die Lagen der Flüssigkeits-Maxima an und die in derselben Spalte rechts davon stehenden zwei Zahlen die Lagen des 1. Maximums. Die Intensitätskurve von geschmolzenem $\mathrm{Bi}$ in $\mathrm{Abb} .8$ a (untere Kurve) zeigt in aller Deutlichkeit, daß das 1. Maximum durch Überlagerung verschiedener Beugungsvorgänge zustande kommt. Weiter ist an dieser Kurve ihr steiler Abfall nach der Seite der kleinen $\sin \vartheta / \lambda$-Werte auffallend; er spricht für das Vorliegen von Flächengitter-Interferenzen ${ }^{50}$; vielleicht sind

und bei hexagonalen ebenfalls mit (001) als Basisebene

$$
\begin{aligned}
& d_{(100)}=\frac{a_{\mathrm{H}}}{2} \sqrt{3} ; d_{(110)}=\frac{a_{\mathrm{H}}}{2} \sqrt{3} \cdot \frac{1}{\sqrt{3}} ; \\
& d_{(200)}=\frac{a_{\mathrm{H}}}{2} \sqrt{3} \cdot \frac{1}{\sqrt{4}} ; d_{(210)}=\frac{a_{\mathrm{H}}}{2} \sqrt{3} \cdot \frac{1}{\sqrt{7}} ; \\
& d_{(300)}=\frac{a_{\mathrm{H}}}{2} \sqrt{3} \cdot \frac{1}{\sqrt{9}} \text { usf., }
\end{aligned}
$$

d. h. die $d$-Werte verhalten sich hier wie

$$
1: 1 / \sqrt{3}: 1 / \sqrt{4}: 1 / \sqrt{7}: 1 / \sqrt{9} \text { usf. }
$$

Die Flächengitter-Interferenzen folgen im hexagonalen Falle weniger dicht als im kubischen aufeinander. - Bei Flächengittern orthogonaler Systeme mit der (001)-Ebene als Basisfläche sind nur Interferenzen von den Ebenen $(h k 0)$, also mit der [001]-Richtung als Zonenachse, möglich. Bei Schichtpaketen mit Flächengitter-Charakter tritt zusätzlich noch die Raumgitter-Reflexion (001) $n$ auf. Für 


\begin{tabular}{|c|c|c|c|c|c|c|c|c|c|}
\hline \multicolumn{3}{|c|}{$\frac{\left(\frac{\sin \theta_{\nu}}{\lambda}\right)_{\text {exp. }}}{\text { nach CHAMBERLAIN }{ }^{24} \text { bei } 310^{\circ} \mathrm{C}}$} & \multicolumn{3}{|c|}{$\begin{array}{l}\left(\frac{\sin \vartheta_{\nu}}{\lambda}\right)_{\exp .} \\
\text { nach SHARRAH } \\
\text { u. SMITH }^{11}\end{array}$} & \multirow{2}{*}{$\begin{array}{c}\left(\frac{\sin \theta_{v}}{\lambda}\right)_{\mathrm{Fl}} \\
\begin{array}{c}\text { berechnet } \\
\text { aus dem } \\
\text { mum }\end{array} \\
0,182\end{array}$} & \multicolumn{2}{|c|}{$\begin{array}{c}\left(\frac{\sin \vartheta_{\nu}}{\lambda}\right)_{(k k 0)}^{\text {Flg. }} \\
\text { experimentell nach CHAMBERLAIN }{ }^{24}\end{array}$} & \multirow{2}{*}{$\begin{array}{c}\left(\frac{\sin \vartheta_{\nu}}{\lambda}\right)_{(h k 0)}^{\text {Flg. }} \\
\begin{array}{c}\text { berechnet aus der } \\
(210)-\text { Interferenz } \\
\text { nach SHARRAH } \\
\text { u. SMITH }{ }^{11}\end{array} \\
0,164\end{array}$} \\
\hline I. Max. & $\approx 0,188$ & $\begin{array}{l}-0,164 \\
--0,160\end{array}$ & $\begin{array}{l}300^{\circ} \mathrm{C} \\
550^{\circ} \mathrm{C}\end{array}$ & $\begin{array}{r}0,182 \\
0,184\end{array}$ & $\begin{array}{l}0,175 \\
0,170\end{array}$ & & $(100)$-Interferenz & - & \\
\hline II.Max. & \multicolumn{2}{|c|}{$\begin{array}{l}0,335 \\
0,336\end{array}$} & \multicolumn{3}{|c|}{$\begin{array}{l}0,33 \\
0,33\end{array}$} & $0,330^{*}$ & (110)-Interferenz & 0,239 & 0,232 \\
\hline III. Max. & \multirow{2}{*}{\multicolumn{2}{|c|}{$\begin{array}{l}0,516 \\
0,502\end{array}$}} & \multicolumn{3}{|c|}{$\begin{array}{l}0,495 \\
0,495\end{array}$} & 0,479 & $(200)$-Interferenz & - & 0,327 \\
\hline IV. Max. & & & \multicolumn{3}{|c|}{$\begin{array}{l}0,65 \\
0,65\end{array}$} & 0,626 & $(210)$-Interferenz & 0,382 & $0,366^{*}$ \\
\hline
\end{tabular}

Tab. 2. Experimentelle und berechnete Lagen für die Intensitäts-Maxima und für die Flächengitter-Interferenzen ( $h k 0)$ bei geschmolzenem $\mathrm{Bi}\left[{ }^{*}\right.$ Berechnung zugrunde gelegter Wert]

die vielen kleinen Maxima der Streukurve als Interferenzen eines Flächengitters zu deuten. Faßt man das kleine Maximum bei $\sin \vartheta / \lambda=0,366$ auf dem abfallenden Ast des II. Maximums der KugelmodellAnordnung als die (210)-Interferenz eines Flächengitters bzw. eines sehr dünnen Schichtpaketes mit Flächengitter-Charakter von einfach-kubischer Struktur ${ }^{53}$ mit $a_{\text {Flg. }}=3,06 \AA$ auf, so besitzen nach Tab. 2 (letzte Spalte) die weit stärkeren Flächengitter-Interferenzen (100), (110) und (200) die in Abb. 8 a durch die Pfeile $\downarrow$ markierten Lagen. Danach sind die schwachen Maxima der Intensitätskurve offenbar als Interferenzen eines Flächengitters ${ }^{54} \mathrm{zu}$ deuten. Der steile Abfall des 1. Maximums der $300{ }^{\circ} \mathrm{C}$ Kurve ist somit durch die (100)-Flächengitter-Interferenz hervorgerufen. Bei Vorliegen von dünnen Schichtpaketen wird die (100)-Flächengitter-Interferenz durch die (001)-Raumgitter-Interferenz noch verstärkt; denn für die Winkellage der beiden Inter-

die Winkellagen dieser Interferenzen gilt im kubischen Fall

$$
(\sin \vartheta / \lambda)_{(100) n}^{\mathrm{Flg} .}=(\sin \vartheta / \lambda) \underset{(001) n}{\mathrm{Rg} .}
$$

und, wie später gezeigt wird, im hexagonalen Falle bei dichtester Kugelpackung

$$
(\sin \vartheta / \lambda)_{(100)}^{\text {Flg. }}<(\sin \vartheta / \lambda) \underset{(001) 2}{\mathrm{Rg} .} .
$$

In allen anderen kubischen Fällen, also mit einer höher indizierten Ebene als Basisfläche, lassen sich die Indizes der zur Zonenachse $[u, v, w]$ gehörigen Netzebenen unmittelbar aus der Zonengleichung $h u+k v+l w=0$ erhalten.

51 M. v. LaUe, Z. Krist. 82, 127 [1932].

52 B. E. WARren, Phys. Rev. 59, 693 [1941].

53 Aus der Lage der (210)-Flächengitter-Interferenz kann man die Größe $a_{\mathrm{Flg}}=a_{\mathrm{Rg}}$. berechnen und hieraus die Lagen der weiteren Flächengitter-Interferenzen $(h k 0)$ mit (001) als Basisfläche; im einfach-kubischen Falle gilt nämlich $\sin \vartheta_{(210)} / \lambda=1 / 2 d_{(210)}=\sqrt{5} / 2 a_{\text {Flg. . }}$.

${ }^{54}$ Die Deutung als Raumgitter-Interferenzen von $\mathrm{Bi}$ entfällt ferenzen gilt im vorliegenden Falle (vgl. Tab. 2)

$$
\left(\frac{\sin \vartheta}{\lambda}\right)_{(100)}^{\mathrm{Flg} .}=\left(\frac{\sin \vartheta}{\lambda}\right)_{(001)}^{\mathrm{Rg} .}=0,164,
$$

d. h. die Flächengitter-Interferenz (100) und die Raumgitter-Interferenz (001) treten unter demselben Winkel auf. Das 1. Maximum der $300^{\circ} \mathrm{C}$-Kurve in Abb. 8 a kann also durch Superposition der

a) Flächengitter-Interferenz (100) der Schicht-

$\beta$ ) Raumgitter-Interferenz (001) $\}$ pakete,

$\gamma$ ) I. Flüssigkeits-Interferenz der dichtesten Atompackung (statistisches Kugelmodell)

hervorgebracht sein. Die Flüssigkeits-Interferenzen entstehen indessen durch Beugung an kleinsten Bereichen mit dichtester Atomanordnung nach Art des statistischen Kugelmodells; sie sind nach Debye ${ }^{55}$, BoERSCH ${ }^{56}$ und $\mathrm{W}_{\text {ARREN }}{ }^{57}$ zu berechnen. Für die Winkellage der obigen drei Interferenzen besteht die Gleichung 58

auch deshalb, weil sich die stärkste (innerste) Gitter-Interferenz (102) mit $\sin \vartheta / \lambda=0,153$ im Streudiagramm der Abb. 8 nicht abzeichnet; Ähnliches gilt auch für das flüssige $\mathrm{Sb}$ (vgl. Tab. 3).

55 P. Debye, Ann. Phys., Lpz. 46, 809 [1915].

56 H. Boersch, Z. Phys. 119, 154 [1942].

57 B. E. W ARren, Z. Krist. 86, 349 [1933].

58 Diese Beziehung gilt immer in einfach-kubischen Fällen. Für den Fall dichtester Atompackung ist $r_{1}>a_{\mathrm{kub} .}^{\mathrm{Flg}}$. Mithin gilt $\left(r_{1}=\right.$ kürzester Atomabstand bei dichtester Atompackung)

$$
\left(\frac{\sin \vartheta}{\lambda}\right)_{(100) \text { kub. }}^{\text {Flg. }}=\frac{1}{2 d_{(100) \mathrm{kub} .}}=\frac{0,5}{a_{\mathrm{kub} .}^{\mathrm{Flg}}}<\frac{0,615}{r_{1}}
$$

Der Unterschied der Zählerwerte beträgt mehr als $18 \%$. Beim Übergang von der Vierer- zur Zwölfer-Koordination und der damit verbundenen Atomradien-Vergrößerung von $\approx 12 \%$ (Änderung der Nennerwerte) kann der Unterschied von $18 \%$ in den Zählerwerten nicht ausgeglichen werden. In den betrachteten (einfach-kubischen) Fällen gilt somit die obige Ungleichung. 


$$
\left(\frac{\sin \vartheta}{\lambda}\right)_{(100)}^{\mathrm{Flg} .}=\left(\frac{\sin \vartheta}{\lambda}\right)_{(001)}^{\mathrm{Rg} .}<\frac{\sin \vartheta_{\mathrm{I}}}{\lambda}=0,615 \cdot \frac{1}{r_{1}}
$$

d. h. die Flächengitter-Interferenz (100) und die (001)-Raumgitter-Interferenz verlagern das I. Flüssigkeits-Maximum nach kleinen Werten von $\sin \vartheta / \lambda$.

In der $300{ }^{\circ} \mathrm{C}$-Kurve von Abb. 8 a wird der Verlauf des 1. Maximums z. Tl. von der (100). Flächengitter-Interferenz bestimmt. Mit steigender Temperatur treten die Flächengitter-Interferenzen, wie die $550^{\circ} \mathrm{C}$-Kurve zeigt, zugunsten des I. Flüssigkeits-Maximums zurück. Aus der überragenden Höhe des 1. Maximums der $550{ }^{\circ} \mathrm{C}$-Kurve gegenüber der des I. Flüssigkeits-Maximums, markiert durch den Pfeil $\bar{\downarrow}$, ist zu schließen, daß noch immer Schichtpakete mit Flächengitter-Charakter im Spiele sind, obwohl der Abfall der Intensitätskurve jetzt weniger steil ist. Dieser Befund ist verständlich, wenn man bedenkt, da $\beta$ nach $W_{\text {ARREN }}{ }^{52}$ das Intensitätsmaximum der Flächengitter-Interferenzen $(h k 0)$ bei konstantem $\lambda$ nach um so größeren Winkeln verschoben ist, je kleiner die Ausdehnung des Flächengitters ist. $\mathrm{Da}$ in den Metallschmelzen die Flächengitter-Bereiche (Schichtpakete) insbesondere bei höheren Temperaturen von recht unterschiedlicher Größe sind, geht auf den zugehörigen Streubildern das charakteristische Aussehen eines Flächengitter-Diagrammes verloren. Die obere Kurve der Abb. 8 a läßt dennoch deutlich erkennen, da $\beta$ das 1 . Maximum $\left(\sin \vartheta_{1} / \lambda\right.$ $=0,170$ ) durch Überlagerung der (100)-Flächengitter-Interferenz $(\sin \vartheta / \lambda=0,164)$ mit dem I. Flüssigkeits-Maximum $\left(\sin \vartheta_{\mathrm{I}} / \lambda=0,182\right)$ zustande kommt. Bei noch höheren Temperaturen verschwindet schließlich auch die (100)-Flächengitter-Interferenz, und gleichzeitig sinkt die Höhe des 1. Maximums der Streukurve auf die des I. Flüssigkeits-Maximums herab. Nach Abb. 8 a scheint unmittelbar am Schmelzpunkt die Schichtpaket-Struktur, von ihm weit entfernt dagegen die Flüssigkeits-Struktur vorherrschend zu sein. Das Nebeneinander von Paket-Struktur mit z. Tl. Flächengitter-Charakter $\left(N_{1}=6\right.$ bzw. 4$)$ und Flüssigkeits-Struktur $\left(N_{1}=12\right)$ in geschmolzenem Bi setzt die Atomzahl auf $N_{1}=7$ bis 8 herab $^{59}$. Aus dieser Erniedrigung von $N_{1}$ kann man auf das Verhältnis von Paket- zu Flüssigkeits-Struktur schlie-

59 Eine Abweichung der Atomzahl $N_{1}$ von der Zwölfer-Koordination deutet offensichtlich darauf hin, daß noch andere Atomgruppierungen vorliegen, ebenso die Verschiebung des I. Flüssigkeits-Maximums nach kleinen $\sin \vartheta / \lambda$ Werten. ßen. Man findet hierfür einen Wert von etwa $1: 2$, in Übereinstimmung mit den verschiedenen Intensitätsbeiträgen zum 1. Maximum in der unteren Streukurve der Abb. 8 a.

Bestätigt werden diese Vorstellungen vom Aufbau der Bi-Schmelze durch eine nur wenig ältere Arbeit von Chamberlain ${ }^{24}$. In Abb. $8 \mathrm{~b}$ ist eine Aufnahme von $\mathrm{Bi}$ aus dieser Arbeit wiedergegeben. Die ausgezogene und die gestrichelte Kurve stellen zwei Intensitäts-Kurven von geschmolzenem $\mathrm{Bi}$ dar. Die Kreise mit der Schwankungsbreite $\bigcirc$ für ihre Lagen beziehen sich auf die ausgezogene Kurve. Bei einem Vergleich mit Abb. 8 a treten in Abb. 8 b die Flächengitter-Interferenzen (100) und (210) besonders stark hervor; daneben zeichnet sich in der ausgezogenen Kurve, allerdings schwach, auf dem abfallenden Ast des 1. Maximums auch die I. Flüssigkeits-Interferenz von $\mathrm{Bi}$ bei $\left(\sin \vartheta_{\mathrm{I}} / \lambda\right)_{\text {exp. }} \approx 0,188$ ab. Die aus Abb. $8 \mathrm{~b}$ erhaltenen $\sin \vartheta / \lambda$-Werte für die Flüssigkeits- und Flächengitter-Interferenzen sind ebenfalls in Tab. 2 zusammengestellt. Die Intensitätskurven in Abb. $8 \mathrm{~b}$ lassen den Flächengitter-Charakter besonders deutlich erkennen, deshalb tritt hier das 1. Maximum $\left[\left(\sin \vartheta_{1} / \lambda\right)_{M}=0,162\right]$ ganz in der Nähe der (100)-Flächengitter-Interferenz mit $(\sin \vartheta / \lambda)_{(100)}^{\text {Flg. }}=0,164$ auf (vgl. Tab. 2). In Abb. $8 \mathrm{a}$ ist dagegen die Flächengitter-Struktur etwas weniger ausgeprägt, daher ist hier das 1. Maximum mehr zwischen den $\sin \vartheta / \lambda$-Werten für die Flüssigkeitsund Flächengitter-Struktur zu beobachten, d. h. nach Tab. 2 zwischen $\sin \vartheta_{\mathrm{I}} / \lambda=0,182$ und $\left(\sin \vartheta_{1} / \lambda\right)_{(100)}^{\mathrm{Flg} .}$ $=0,164$.

In Abb. 8 liegt weitgehend ein Überlagerungsdiagramm vor. Zur Einzeichnung in Abb. 7 kann daher nicht der mittlere (experimentelle) Wert von $\left(\sin \vartheta_{1} / \lambda\right)_{\mathrm{M}}=0,168$ benützt werden, vielmehr muß hierzu der aus dem II. Flüssigkeits-Maximum errechnete Mittelwert $\left(\sin \vartheta_{\mathrm{I}} / \lambda\right)_{\mathrm{M}}=0,182$ herangezogen werden (vgl. Tab. 1). Wie oben bereits bemerkt, erhält man aus Abb. 7 als Atomradius für die Flüssigkeits-Struktur (wahrscheinlich: Zwölfer-Koordination) $a_{\text {ber. }}^{*}=1,68 \AA$ und für die einfach-kubische Paket-Struktur $a_{\text {ber. }}^{*}=1,53 \AA$, also Werte, die erheblich kleiner als der in der Literatur aufgeführte Atomradius für die Zwölfer-Koordination von $\mathrm{Bi}$ $\left(a_{12}{ }^{*}=1,82 \AA\right)$ sind; vielleicht findet in dieser starken Verringerung des kürzesten Atomabstandes die Schmelz-Anomalie von Bi ihre Begründung. 


\section{b) Antimon}

Auf der gleichen Basis läßt sich die Streukurve von geschmolzenem $\mathrm{Sb}\left(\mathrm{Schmelzpunkt}=630^{\circ} \mathrm{C}\right)$ deuten, die kürzlich von Hendus und MülLER 38,39 bei $665^{\circ} \mathrm{C}$ erhalten wurde (vgl. auch MüLLER ${ }^{60}$ ) ; sie ist in Abb. 9 wiedergegeben. Zunächst seien auch hier die Lagen der Flüssigkeits-Maxima berechnet; sie sind in Tab. 3 mit den experimentellen Werten zusammengestellt. Aus dem II. bzw. III. Maximum errechnet man für die Winkellage des I. Flüssigkeits-Maximums $\sin \vartheta_{\mathrm{I}} / \lambda=0,187$ und als kürzesten Atomabstand $r_{1}=3,29 \AA \AA$, der nach Tab. 1 mit dem doppelten Atomradius $a^{*}$ für Zwölfer-Koordination praktisch identisch ist. Hendus und MüLleR ${ }^{39}$ finden dagegen als Ergebnis der Fourier-Analyse der Streukurve von aufgeschmolzenem $\mathrm{Sb} r_{1}=3,12 \AA$ und $N_{1}=6,1$ (vgl. auch Richter und Mitarb. ${ }^{61}$ ).

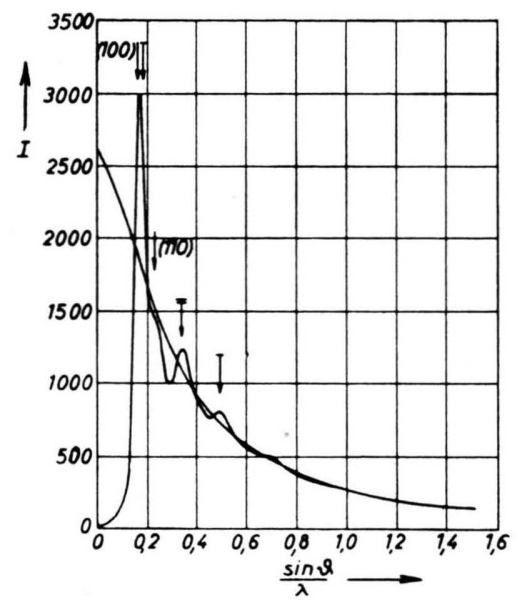

Abb. 9. Intensitätskurve von geschmolzenem Sb.

$\downarrow=$ berechnete Lagen der Flüssigkeits-Maxima,

$\downarrow=$ berechnete Lage der Flächengitter-Interferenz (100).

Die Berechnung der Lagen für die Flächengitter-Interferenzen der Schichtpakete macht hier einige Schwierigkeiten, da nur ein Maximum bei $\sin \vartheta / \lambda \approx 0,232 \mathrm{zu}$ beobachten ist, zudem ist seine Lage schwer zu bestimmen. Wenn auch die Streukurve von flüssigem $\mathrm{Sb}$ in Abb. 9 in nicht so ausgeprägter Form wie die von $\mathrm{Bi}$ in Abb. 8 auf das Vorliegen von Flächengitter-Interferenzen hinweist, so sind doch gewisse Anzeichen wie die Höhe und die Schärfe des 1. Maximums, evtl. auch des 2. Maximums vorhanden, die es angezeigt erscheinen lassen, das kleine Maximum bei $\sin \vartheta / \lambda \approx 0,232$ mit der Flächengitter-Interferenz (110) zu identifizieren; zudem spricht auch hierfür der analoge Verlauf der Streukurven von geschmolzenem Bi und Sb (vgl. die Abb. 8 und 9). Aus der Lage dieses Maximums errechnet man bei Annahme einer einfach-kubischen Struktur

${ }^{60}$ H. K. F. Müller, Dissertation, Universität d. Saarlandes, Juni 1956.

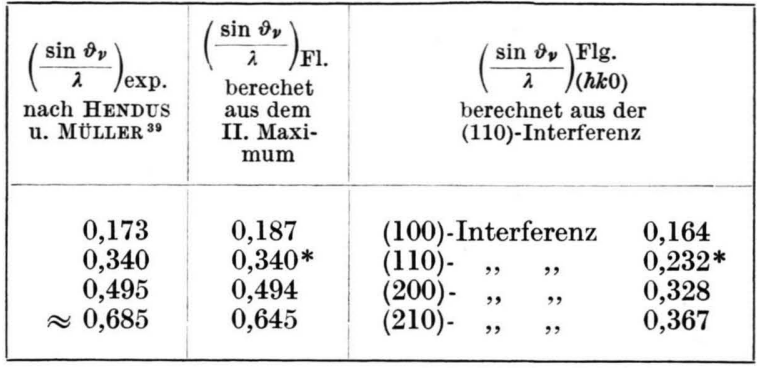

Tab. 3. Experimentelle und berechnete Lagen für die Intensitäts-Maxima und für die Flächengitter-Interferenzen $(h k 0)$ bei geschmolzenem $\mathrm{Sb}$ [ ${ }^{*}$ Berechnung zugrunde gelegter Wert].

als Kantenlänge für das Flächengitter $a_{\mathrm{Flg} .}=3,06 \AA$ und hieraus als Winkellagen für die Flächengitter-Interferenzen $(h k 0)$ die in Tab. 3 angegebenen Werte. Die Lagen dieser Interferenzen und die der FlüssigkeitsMaxima sind in Abb. 9 durch die Pfeile $\downarrow$ bzw. $\downarrow$ gekennzeichnet. Bei Vorliegen von Schichtpaketen ist das 1. Maximum der Intensitätskurve noch durch die (001) Raumgitter-Interferenz mit der gleichen Winkellage wie die Flächengitter-Interferenz (100) angehoben. Bezüglich der Interferenzlagen gilt hier wieder

$$
(\sin \vartheta / \lambda)_{(100)}^{\mathrm{Flg} .}=(\sin \vartheta / \lambda)_{(001)}^{\mathrm{Rg} .}<\sin \vartheta_{\mathrm{I}} / \lambda .
$$

Wie bei flüssigem Bi entsteht offensichtlich auch bei $\mathrm{Sb}$ das 1. Maximum der Intensitätskurve durch Überlagerung verschiedener Beugungsvorgänge. Je nach dem Grade, mit dem die einzelnen Prozesse am Zustandekommen des 1. Maximums der Streukurve beteiligt sind, wird seine Lage zwischen den beiden in Tab. 3 angegebenen Extremwerten streuen. Das II. Maximum der Flüssigkeits-Struktur liegt ähnlich wie bei der Streukurve von geschmolzenem $\mathrm{Bi}$ (vgl. Abb. 8) in der Nähe der beiden Flächengitter-Interferenzen (200) und (210); diese Interferenzen bedingen bei Sb offensichtlich die Schärfe und die besondere Form des II. Maximums. Mit wachsender Temperatur werden auch hier die Raum- und Flächengitter-Interferenzen der Schichtpakete zurücktreten, so daß der Verlauf der Intensitätskurve vorwiegend von der dichtesten Atompackung des statistischen Kugelmodells bestimmt wird; in diesem Falle sollte nach Tab. 1, Spalte 7, das I. Flüssigkeits-Maximum bei $\sin \vartheta_{\mathrm{I}} / \lambda=0,190$ auftreten.

Wegen der kleinen Integrationslänge vermag die Fourier-Analyse der Intensitätskurve dicht beieinanderliegende Abstände nicht zu trennen, sondern liefert als kürzesten Atomabstand bzw. Atomzahl für die unterschiedlichen Anordnungen der Atome (Schichtpakete und statistisches Kugelmodell) einen Mittelwert, der zugunsten der am stärksten vertretenen Struktur verschoben ist. Aus dem Unterschied der berechneten Atomabstände $r_{1}=3,29 \AA$ bzw. $a_{\mathrm{Flg} .}=3,06 \AA$ und dem Mittelwert von Hendus und MüLleR ${ }^{39}\left(r_{1}=3,12 \AA ̊\right.$ und

61 H. Richter, H. Berckhemer u. G. Breitling, Z. Naturforschg. 9 a, 236 [1954]. 


\begin{tabular}{|c|c|c|c|c|c|c|}
\hline Element & $\left(\frac{\sin \vartheta_{1}}{\lambda}\right)_{\exp }$ & $\left(\frac{\sin \theta}{\lambda}\right)_{\mathrm{Nbm}}$ & $d_{1}$ & $d_{\mathrm{Nbm}}$ & $\begin{array}{l}\text { Netzebenenabstände } \\
\text { des Gitters }\end{array}$ & $\begin{array}{l}\text { Autoren s. } \\
\text { Anm. Nr. }\end{array}$ \\
\hline $\mathrm{Hg}$ & $\begin{array}{l}0,181 \\
0,198 \\
0,171 \\
0,183 \\
0,184\end{array}$ & $\begin{array}{l}- \\
\overline{-} \\
0,219 \\
-\end{array}$ & $\begin{array}{l}2,76 \\
2,52 \\
2,92 \\
2,74 \\
2,72\end{array}$ & $\begin{array}{c}- \\
- \\
\overline{2,28} \\
-\end{array}$ & $\begin{array}{l}\text { Rhomboedrisch } \\
(100): 2,75 \text { s. st. } \\
(111))\{2,24 \text { s. st. } \\
(110)\} \\
(101): 1,74 \mathrm{~m} .\end{array}$ & $\begin{array}{l}29 \\
30 \\
31 \\
32 \\
33\end{array}$ \\
\hline In & $\begin{array}{l}0,179 \\
0,181\end{array}$ & - & $\begin{array}{l}2.79 \\
2,76\end{array}$ & - & $\begin{array}{l}\text { Tetragonal } \\
(111): 2,79 \text { s. st. } \\
(200): 2,29 \text { st. } \\
(220): 1,62 \text { st. }\end{array}$ & $\begin{array}{r}20 \\
23 \\
3\end{array}$ \\
\hline Sn & $\begin{array}{l}0,177 \\
0,174 \\
0,177\end{array}$ & $\begin{array}{l}-\overline{232}+ \\
0,240\end{array}$ & $\begin{array}{l}2,83 \\
2,87 \\
2,83\end{array}$ & $\begin{array}{l}\overline{2,16} \\
2,08\end{array}$ & $\begin{array}{l}\text { Tetragonal } \\
(200): 2,91 \text { s. st. } \\
(101): 2,79 \text { s. st. } \\
(220): 2,06 \mathrm{~m} . \\
(121): 2,01 \text { st. }\end{array}$ & $\begin{array}{r}37 \\
20 \\
3\end{array}$ \\
\hline $\mathrm{Ga}$ & $\begin{array}{l}0,194 \\
0,199\end{array}$ & $\begin{array}{l}0,243^{+} \\
0,249\end{array}$ & $\begin{array}{l}2,58 \\
2,51\end{array}$ & $\begin{array}{l}2,06 \\
2,01\end{array}$ & $\begin{array}{l}\text { Rhombisch } \\
(002): 3,83 \mathrm{~s} . \\
(111): 2,96 \mathrm{~s} . \text { st. } \\
(102): 2,92 \mathrm{~s} . \text { st. } \\
(200)\} 2,25 \mathrm{~m} . \\
(020)\} \text { st. } \\
(113): 1,99 \mathrm{st} \\
(211): 1,96 \mathrm{~m} .\end{array}$ & $\begin{array}{r}31 \\
3\end{array}$ \\
\hline $\mathrm{Ge}$ & 0,198 & 0,260 & 2,52 & 1,92 & $\begin{array}{l}\text { Kubisch } \\
(111): 3,25 \text { s. st. } \\
(220): 1,99 \text { st. } \\
(311): 1,70 \text { st. }\end{array}$ & 3 \\
\hline $\mathrm{Sb}$ & 0,173 & $0,232^{+}$ & 2,89 & 2,16 & $\begin{array}{l}\text { Hexagonal } \\
(003): 3,75 \mathrm{~s} . \\
(101): 3,54 \mathrm{~s} . \mathrm{s} . \\
(102): 3,15 \mathrm{~s} . \text { st. } \\
(104): 2,24 \mathrm{st} . \\
(110): 2,15 \mathrm{st} . \\
(105): 1,92) \mathrm{s} .\end{array}$ & 38,39 \\
\hline $\mathrm{Bi}$ & $\begin{array}{l}0,170 \\
0,166 \\
0,175 \\
0,162\end{array}$ & $\begin{array}{l}-\overline{2} \\
0,20 \\
0,232^{+} \\
0,239^{+}\end{array}$ & $\begin{array}{l}2,94 \\
3,01 \\
2,86 \\
3,09\end{array}$ & $\begin{array}{l}-\overline{2} \\
2,16 \\
2,09\end{array}$ & $\begin{array}{l}\text { Hexagonal } \\
(003): 3,95 \mathrm{~s} . \\
(101): 3,73 \mathrm{~s} . \mathrm{s} . \\
(102): 3,27 \mathrm{s.} \text { st. } \\
(104): 2,36 \mathrm{st} . \\
(110): 2,27 \mathrm{st} . \\
(105): 2,03 \mathrm{~s} .\end{array}$ & $\begin{array}{r}22 \\
3 \\
11 \\
24\end{array}$ \\
\hline
\end{tabular}

+ Der Intensitätskurve entnommene Werte.

Tab. 4. Zuordnung der Periodenwerte $d_{1}$ und $d_{\mathrm{Nbm}}$ zu Netzebenenabständen des jeweiligen Gitters.

$\left.N_{1}=6,1\right)$ läßt sich näherungsweise ${ }^{62}$ das Verhältnis von Paket- zu Flüssigkeits-Struktur abschätzen, ebenso aus den erhaltenen Atomzahlen.

\section{c) Germanium, Gallium, Zinn und Indium}

Die Intensitätskurven von $\mathrm{Ge}, \mathrm{Ga}$ und $\mathrm{Sn}$ besitzen auf dem abfallenden Ast des 1. Maximums wie die von $\mathrm{Bi}$ und $\mathrm{Sb}$ ein kleines Nebenmaximum ${ }^{63}$. Die Lage

${ }^{62}$ Durch die spezifische Intensitätsverteilung der (100)-Flächengitter-Interferenz wird das I. Flüssigkeits-Maximum besonders stark nach kleinen Winkelwerten verschoben, so daß das hieraus ermittelte Komponenten-Verhältnis bezüglich der Paket-Struktur zu groß erhalten wird. dieses Nebenmaximums und die des 1 . Maximums sind in Tab. 4 für die oben genannten Elemente sowie für $\mathrm{Hg}$, In, Sb und $\mathrm{Bi}$ zusammengestellt, ebenso die aus der BRAGgschen Beziehung sich hierfür ergebenden Periodenwerte $d_{\mathrm{Nbm}}$. und $d_{1}$. Weiter sind in dieser Tabelle die ersten Netzebenen-Abstände für die obigen Gitter angegeben. Nach Tab. 4 ist eine Zuordnung der $d_{\text {Nbm. }}$-Werte zu gewissen Netzebenen-Abständen des jeweiligen Gitters z. Tl. gut möglich. Auffallend ist

${ }^{63} \mathrm{Das}$ von Hendus ${ }^{32}$ bei flüssigem $\mathrm{Hg}$ beobachtete schwache Maximum mit $\sin \vartheta / \lambda=0,219$ konnte nicht bestätigt werden. 


\begin{tabular}{|c|c|c|c|c|c|c|c|}
\hline Element & $\left(\frac{\sin \theta_{1}}{\lambda}\right)_{\exp }$ & $\begin{array}{c}\left(\frac{\sin \vartheta_{1}}{\lambda}\right) \\
\text { berechnet } \\
\text { aus dem } \\
\text { II. Maximum }\end{array}$ & $\frac{\sin \vartheta_{\mathrm{Nbm} .}}{\lambda}=\left(\frac{\sin \vartheta}{\lambda}\right)_{(110)}^{\mathrm{Flg} .}$ & $\left(\frac{\sin \theta}{\lambda}\right)_{(100)}^{\text {Flg. }}$ & $a_{\text {Flg. }}$ & $2 a_{12} *$ & $\begin{array}{l}\text { Autoren siehe } \\
\text { Anm. Nr.' }\end{array}$ \\
\hline Sn & $\begin{array}{l}0,174 \\
0,177\end{array}$ & $\begin{array}{l}0,184 \\
0,186\end{array}$ & $\begin{array}{l}0,232 \\
0,240\end{array}$ & $\begin{array}{l}0,164 \\
0,170\end{array}$ & $\begin{array}{l}3,05 \\
2,95\end{array}$ & 3,16 & $\begin{array}{l}20 \\
3\end{array}$ \\
\hline $\mathrm{Ga}$ & $\begin{array}{l}0,194 \\
0,199\end{array}$ & $\begin{array}{l}0,207 \\
0,214\end{array}$ & $\begin{array}{l}0,243 \\
0,249\end{array}$ & $\begin{array}{l}0,172 \\
0,176\end{array}$ & $\begin{array}{l}2,91 \\
2,84\end{array}$ & 2,78 & $\begin{array}{l}31 \\
3\end{array}$ \\
\hline $\mathrm{Ge}$ & 0,198 & 0,215 & 0,260 & 0,184 & 2,72 & 2,78 & 3 \\
\hline
\end{tabular}

Tab. 5 a. Experimentelle und berechnete Lagen für $\sin \vartheta_{1}, \vartheta_{\mathrm{I}}, \vartheta_{\mathrm{Nbm}} / \lambda$ sowie für die Flächengitter-Interferenzen (100) und (110) bei geschmolzenem $\mathrm{Sn}, \mathrm{Ga}$ und $\mathrm{Ge}$.

dabei, daß die Periodenwerte $d_{\mathrm{Nbm}}$. mit den NetzebenenAbständen von (110) bzw. (220) weitgehend übereinstimmen, ausgenommen bei $\mathrm{Ga}$. Dieser Befund könnte als Hinweis dafür dienen, daß in der Schmelze noch kleinste Gitterbereiche vorhanden sind. Andererseits ist nach Tab. 1 (vgl. Spalte 7 bzw. 6 mit 3) das I. Flüssigkeits-Maximum von geschmolzenem $\mathrm{Ge}, \mathrm{Ga}$ und $\mathrm{Sn}$ zum Teil beträchlich nach kleinen $\sin \vartheta / \lambda$-Werten verschoben. Versucht man diese Verschiebung durch die vorderen Gitter-Interferenzen zu erklären, so hat eine solche Deutung, wie die eingehende Prüfung zeigt, nicht bei allen Elementen den gewünschten Erfolg.

Unter diesen Umständen liegt es nahe, das Nebenmaximum auf dem abfallenden Ast des 1. Maximums wie bei geschmolzenem $\mathrm{Bi}$ und $\mathrm{Sb}$ als (110)-Flächengitter-Interferenz eines Schichtpaketes ${ }^{64}$ mit einfachkubischer ${ }^{67}$ Struktur und der Kantenlänge $a_{\text {Flg. }}$ zu deuten (vgl. Tab. 5 a). Für das Vorliegen einer Flächengitter-Struktur spricht der bei $\mathrm{Ga}$ und $\mathrm{Sn}$ zu beobachtende steile Abfall des 1. Maximums der Intensitätskurve nach der Seite der kleinen $\sin \vartheta / \lambda$-Werte (vgl. Hendus $^{3}$ ). Die Verschiebung des I. Flüssigkeits-Maximums wäre danach wie bei flüssigem $\mathrm{Bi}$ und $\mathrm{Sb}$ durch die (100)-Flächengitter-Interferenz zu erklären. So zeigt Tab. 5 a, daß auch hier das 1. Maximum der Intensitätskurve zwischen den berechneten Interferenzlagen von $\sin \vartheta_{\mathrm{I}} / \lambda$ und $(\sin \vartheta / \lambda)_{(100)}^{\mathrm{Flg} \text {. }}$ auftritt.

Beim geschmolzenen Sn stimmt die aus der Lage des Nebenmaximums berechnete Kantenlänge $\bar{a}_{\text {Flg. }}=3,00 \AA$

${ }^{64}$ Im Streubild von geschmolzenem Sn tritt nach Richter ${ }^{65}$ (Elektronenbeugungsversuche) und Hendus ${ }^{3}$ eine schwache Interferenz (Nebenmaximum) mit $d_{\mathrm{Nbm}} \approx 2,08 \AA$ auf; sie wird nach Richter ${ }^{66}$ auch bei Ätzversuchen an Sn erhalten. Dieser Ring ist nach Tab. 5 a als (110)-Flächengitter-Interferenz zu deuten. Demnach wird das Sn-Gitter bereits beim Ätzen in einzelne Schichtpakete abgebaut. Wenn selbst die Metallschmelzen weitgehend Schichtbildung mit Flächengitter-Charakter zeigen, dann ist es nicht verwunderlich, wenn ähnliche Strukturen - allerdings z. Tl. von Metalloxyden - auch bei Ätz-, Polier- und Einlaufvorgängen beobachtet werden. Damit zeigt die Ätz-, Polier- und Einlaufschicht von Sn bereits eine Komponente der Flüssigkeits-Struktur.

65 H. Richter, Phys. Z. 44, 406 [1943].

${ }^{66}$ H. Richter, Z. angew. Phys. 8, 433 [1956]. praktisch mit dem kürzesten Atomabstand im Sn-Gitter $\left(r_{1}\right)_{\mathrm{G}}=3,02 \AA$ überein. Weiter ändert sich nach den Tab. 1 und $5 \mathrm{a}$, und zwar in Übereinstimmung mit der Theorie, der kürzeste Atomabstand beim Ưbergang von der Vierer- bzw. Sechser- zur ZwölferKoordination um etwa 5\%, bei geschmolzenem Sb bzw. Bi um 7 bzw. 10\%. Diese Ergebnisse machen es sehr wahrscheinlich, daß auch im geschmolzenen $\mathrm{Sn}$ eine Überlagerung von einfach-kubischer Schichtpaket- und Kugelmodell-Struktur vorliegt. Dabei ist die einfachkubische Struktur als entzerrtes tetragonales Sn-Gitter aufzufassen. Beim flüssigen $\mathrm{Ga}^{68}$ und $\mathrm{Ge}$ sind die entsprechenden Änderungen des kürzesten Atomabstandes nach den Tab. 1 und 5 a erheblich geringer, und zudem fehlt bei $\mathrm{Ge}$ in Abb. 10 a der steile Intensitätsabfall des 1. Maximums nach der Seite der kleinen $\sin \vartheta / \lambda$-Werte (vgl. Hendus ${ }^{3}$ ); Ga und Ge lassen sich also nur sehr schwer in das Schema der Tab. 5 a einordnen.

Bei flüssigem Ga kann das Nebenmaximum mit der Winkellage $\sin \vartheta_{\mathrm{Nbm}} / \lambda=0,246$ auch durch den Atomabstand im einzelnen Molekül hervorgerufen sein, der sich im vorliegenden Falle nach $\sin \vartheta_{\mathrm{Nbm} .} / \lambda=0,615 \cdot 1 / r_{1}$ zu $r_{1}=2,50 \AA$ berechnet (vgl. Tab. $5 \mathrm{~b}$ ). Dieser Abstandswert stimmt mit dem Atomabstand im EinzelMolekül des Ga-Gitters ${ }^{70}\left[\left(r_{1}\right)_{\mathrm{G}}=2,45 \AA\right.$ und $\left.N_{1}=1\right]$ recht gut überein. Ungeklärt bleibt aber die Verschiebung des I. Flüssigkeits-Maximums, das von der Kugelmodell-Struktur der Einzelatome herrührt.

Versucht man beim geschmolzenen Ge das Nebenmaximum bei $\sin \vartheta_{\mathrm{Nbm}} / \lambda=0,260$ (vgl. Abb. $10 \mathrm{a}$ )

67 Bei Annahme von Flächengittern mit dichtester Atompackung $\quad\left[(111)\right.$ kub. $^{-}=(001)$ hex.-Ebene $=$ Basisebene $]$ würden die (100)- und (110)-Flächengitter-Interferenzen in dem zu großen Abstand $1: \sqrt{3}$ statt $1: \sqrt{2}$ aufeinander folgen (vgl. Anm. ${ }^{50}$ ). Die annähernd gleichen Lagen der Flächengitter-Interferenzen (100) und (110) bezüglich des I. Flüssigkeits-Maximums sind bei $\mathrm{Bi}, \mathrm{Sb}$ und $\mathrm{Sn}$ zu beachten.

68 Die in der Literatur angegebenen Atomradien-Werte für Ga streuen stark. So gibt z. B. Goldschmidt an $a_{12}{ }^{*}=1,39 \AA$, $\mathrm{P}_{\text {AULING }}{ }^{69}$ indessen $a_{12}{ }^{*}=1.53 \AA$ und experimentell findet man im Mittel $a_{12}{ }^{*}=1,46 \AA$.

69 J. E. Hiller, Grundriß der Kristallchemie, Walter de Gruyter, Berlin 1952.

70 A. J. Bradlex, Z. Krist. 91, 302 [1935]. 


\begin{tabular}{|c|c|c|c|}
\hline \multirow[b]{2}{*}{ Element } & \multirow[b]{2}{*}{$\begin{array}{c}\text { Kürzester Gitterabstand } \\
{\left(r_{1}\right)_{\mathrm{G}}}\end{array}$} & Nach DEBYE: & Nach Flächengitter-Vorstellung \\
\hline & & $\left(r_{1}\right)_{\text {Baust. }}=0,615 \frac{1}{\frac{\sin \vartheta_{\mathrm{Nbm}}}{\lambda}}$ & $a_{\text {Flg. }}=d_{(110)} \sqrt{2}=\frac{1}{\sqrt{2} \frac{\sin \vartheta_{\mathrm{Nbm}}}{\lambda}}$ \\
\hline $\mathrm{Sn}$ & 3,02 & - & $\bar{a}_{\text {Flg. }}=3,00$ \\
\hline $\mathrm{Sb}$ & 2,87 & - & $a_{\text {Flg. }}=3,06$ \\
\hline $\mathrm{Bi}$ & 3,10 & - & $\bar{a}_{\text {Flg. }}=3,08$ \\
\hline $\mathrm{Ga}$ & 2,45 & 2,50 & - \\
\hline $\mathrm{Ge}$ & $2,43\left(r_{2}=a_{\mathrm{H}}=3,97\right)$ & 2,37 & $a_{\mathrm{H}}=3,85$ \\
\hline
\end{tabular}

Tab. 5 b. Aus den Lagen der Nebenmaxima $\left(\sin \vartheta_{\mathrm{Nbm}} / \lambda\right.$-Werte) berechnete kürzeste Atomabstände.

\begin{tabular}{|c|c|c|c|c|}
\hline \multirow{2}{*}{$\begin{array}{c}\text { Raumgitter } \\
a_{\text {kub. }}=5,62 \AA\end{array}$} & $\begin{array}{c}\text { Flächengitter } \\
a_{\mathrm{H}}=3,97 \AA\end{array}$ & \multicolumn{2}{|c|}{ Einzel-Tetraeder } & $\begin{array}{c}\text { Flüssigkeits- } \\
\text { Struktur } \\
r_{1}=2,78 \AA\end{array}$ \\
\cline { 3 - 4 } & & $\left(r_{1}\right)_{\text {Tetr. }}=2,43 \AA$ & $\left(r_{2}\right)_{\text {Tetr. }}=3,97 \AA$ & \\
\hline$(111): 0,154$ & $(100): 0,146$ & - & 0,155 & - \\
$(220): 0,252$ & $(110): 0,252$ & 0,253 & 0,282 & 0,221 \\
$(311): 0,295$ & $(200): 0,291$ & 0,460 & 0,408 & 0,403 \\
$(400): 0,356$ & $(210): 0,385$ & 0,667 & 0,535 & 0,584 \\
$(331): 0,388$ & $(300): 0,436$ & 0,873 & 0,661 & 0,764 \\
$(422): 0,436$ & $(220): 0,504$ & 1,08 & 0,787 & 0,943 \\
\hline
\end{tabular}

Tab. 5 c. Interferenzlagen bei Ge $(\sin \vartheta / \lambda$-Werte).

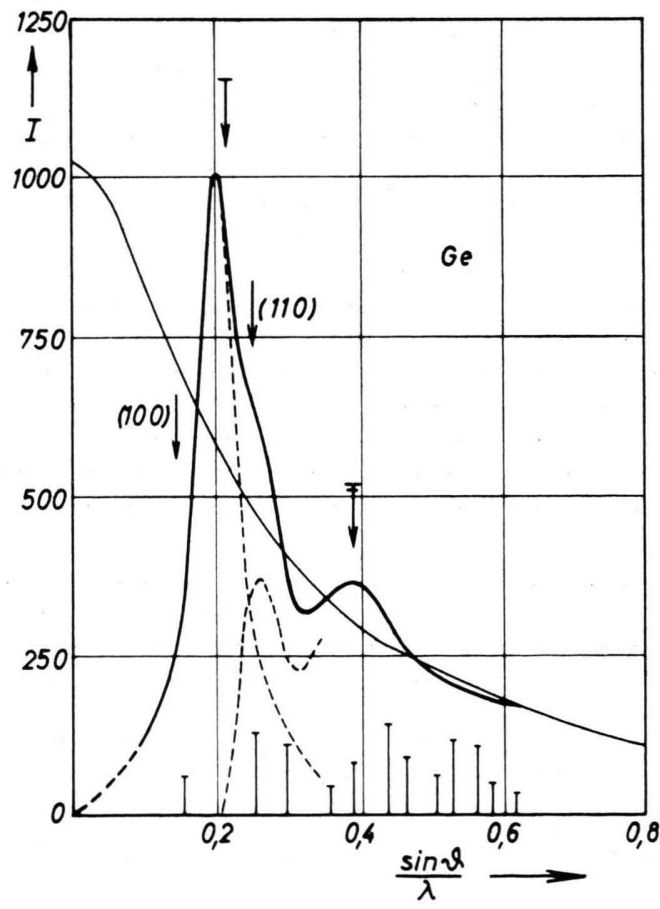

Abb. 10 a. Intensitätskurve von geschmolzenem Ge.

$\downarrow=$ berechnete Lagen der Flüssigkeits-Maxima,

$\downarrow=$ berechnete Lage der Flächengitter-Interferenz (100). ebenfalls nach der DeByeschen Interferenz-Funktion zu deuten, so erhält man als kürzesten Atomabstand $r_{1}=2,37 \AA$. . - Dieser Abstandswert stimmt mit dem kürzesten Atomabstand des Ge-Gitters [Zentralabstand eines regulären Tetraeders mit $\left(r_{1}\right)_{\text {Tetr. }}=2,43 \AA$ und $\left.N_{1}=4\right]$ gut überein. Falls in geschmolzenem Ge EinzelTetraeder vorliegen, dann sollte sich auch die TetraederKante mit $\left(r_{2}\right)$ Tetr. $=3,97 \AA$ abzeichnen, und zwar nach Tab. 5 c bei $\sin \vartheta / \lambda=0,155$. Diese Winkellage fällt mit dem ersten vertikalen Strich in Abb. 10 a, der die Lage der (111)-Interferenz des Ge-Gitters angibt, zusammen.

Nach den Erfahrungen bezüglich des Aufbaues fester amorpher Stoffe schließen sich die Grundbausteine durch Verkettung aneinander. Die Ge-Tetraeder wären demnach wie im Gitter mit allseitiger Verknüpfung aneinander zu fügen, d. h. es müßte sich im flüssigen Ge neben der Kugelmodell-Anordnung in kleinsten Bereichen das normale Gitter, allerdings mit Störungen in seinem Aufbau, ausbilden. Das breite, von Hendus ${ }^{3}$ bei $\sin \vartheta / \lambda=0,26$ beobachtete Nebenmaximum wäre demnach als (220)-Raumgitter-Interferenz mit $\sin \vartheta_{(220)} / \lambda$ $=0,252 \mathrm{zu}$ deuten (vgl. Tab. 4 bzw. 5 c). Die stärkste Gitter-Interferenz von $\mathrm{Ge}$ bei $\sin \vartheta_{(111)} / \lambda=0,154$ hätte somit die starke Verbreiterung des 1. Maximums in Abb. 10 a zur Folge. Entsprechend dem deutlichen Hervortreten der (220)-Gitter-Interferenz müßte die Raumgitter-Struktur in geschmolzenem $\mathrm{Ge}$ noch recht ausgeprägt vorhanden sein. Das starke Zurückbleiben der (111)-Interferenz ist dann allerdings unverständlich; es kann auch nicht durch eine verminderte Meßgenauig. keit infolge erhöhter Absorption in diesem Winkelbereich 
erklärt werden. Nimmt man aber an, daß die RaumgitterBereiche sehr dünn sind, dann wirken sie wie Flächengitter mit der (111) kub.-Ebene bzw. (001) hex.-Ebene als Basis; d. h. es liegen Schichtpakete aus (111) - bzw. (001)-Ebenen vor. Bei $a_{\mathrm{H}}=\left(r_{2}\right)$ Tetr. $=3,97 \AA$ erhält man als Lagen für die Flächengitter-Interferenzen

$(\sin \vartheta / \lambda)_{(100)}=0,146, \quad(\sin \vartheta / \lambda)_{(110)}=0,252$ usf. Diese Lagen, in Abb. 10 a durch die Pfeile $\downarrow$ markiert (Lagen der Flüssigkeits-Maxima $=\downarrow$ ), sind nach Tab. 5 c praktisch mit denen der Raumgitter-Interferenzen von Ge identisch. Weiter sei in Anlehnung an das GeGitter angenommen, daß sich die Schichtpakete aus Doppelschichten mit den Anfangslagen (000) und $\left(\frac{1}{3} \frac{2}{3} z\right)$ aufbauen. In diesen Doppelschichten ist jedes Atom an drei nächste Nachbarn gebunden, sein vierter Nachbar liegt in der benachbarten Doppelschicht. Diese spezielle Atomanordnung führt in der Ge-Schmelze, of fenbar durch den Vorgang der Gleitung begünstigt, zur Ausbildung von Schichtpaketen aus Doppelschichten und mit Flächengitter-Charakter. Damit ist bei Diskussion der Intensitätsverteilung des zu erwartenden Flächengitter-Diagrammes der Strukturfaktor $S$ für die Doppelschicht mitzuberücksichtigen; er ergibt sich für die innersten Flächengitter-Interferenzen (100) und (110) zu $\left|S_{(100)}\right|^{2}=1 \cdot F_{\mathrm{Ge}^{2}}$ und $\left|S_{(110)}\right|^{2}=4 \cdot F_{\mathrm{Ge}}{ }^{2}$. Bei gleicher Flächenhäufigkeit und bei Berücksichtigung des Atomformfaktors ist der Strukturfaktor für die (110)-Ebene etwa 3 -mal ${ }^{71}$ so groß wie der für die (100)-Ebene. Dieses Ergebnis erklärt das starke Hervortreten der Flächengitter-Interferenz (110) bei $\sin \vartheta / \lambda=0,252$ und damit das Zurückbleiben der (100) -Flächengitter-Interferenz bei $\sin \vartheta / \lambda=0,146$ sowie die starke Verbreiterung und Verlagerung des I. Flüssigkeits-Maximums; andererseits beweist es das Vorliegen von Schichtpaketen aus Doppelschichten mit Flächengitter-Charakter in geschmolzenem Ge. Damit lies: in der Ge-Schmelze eine Überlagerung von Schichtpakı't-Struktur mit Flächengitter-Charakter $\left(N_{1}=4\right)$ und Flüssigkeits-Struktur $\left(N_{1}=12\right)$ vor. Ein geringer Anteil an gestörter Raumgitter-Struktur ist nicht auszuschließen. Diese Überlagerung erklärt die von HenDus ${ }^{3}$ beobachtete Atomzahl $N_{1}=8$ und den zu kleinen Atomabstand $r_{1}=2,70$ Å. Damit führt auch beim aufgeschmolzenen Ge die Deutung seiner Intensitätskurve zu einer Schichtpaket-Struktur wie beim geschmolzenen Bi, $\mathrm{Sb}$ und $\mathrm{Sn}$, allerdings mit dem Unterschied. daß die Atomanordnung des betrachteten Flächengitters als Elementarbereich eine hexagonale Zelle besitzt. Wahrscheinlich ist auch die Streukurve von flüssigem $\mathrm{Ga}$ als Überlagerung von Schichtpaket- und Flüssigkeits-Struktur zu deuten.

Zusammenfassend sind in Tab. $5 \mathrm{~b}$ die bisherigen Ergebnisse bezüglich des Nebenmaximums in den Intensitätskurven von geschmolzenem $\mathrm{Sn}, \mathrm{Sb}, \mathrm{Bi}, \mathrm{Ga}$ und Ge zusammengestellt. Hier sind lediglich die kürzesten

71 Berechnet man nach DeBYE ${ }^{55}$ den Verlauf der Streukurve für die Ge-Doppelschicht. so ist für $N^{*}=10$ (maximale Ausdernung $\approx 8 \AA \AA$ ) das erste und zweite Maximum von gleicher Höhe. Mit Ausdehnung des betrachteten Bereiches wird dieses Verhältnis kleiner.
Atomabstände aufgeführt, wie man sie nach der Debyeschen Interferenz-Theorie oder nach der FlächengitterVorstellung aus der jeweiligen Lage des Nebenmaximums errechnet. Bei $\mathrm{Ge}$ und $\mathrm{Ga}$ erhält man nach DeBYe ${ }^{55}$ unmittelbar den kürzesten Gitterabstand. Weiter wurde bei Ge das Nebenmaximum der Streukurve bei $\sin \vartheta / \lambda=0,26$ in Anlehnung an die zugehörige Gitterstruktur als die (110)-Flächengitter-Interferenz eines Schichtpaketes mit hexagonaler Gitterzelle gedeutet (vgl. Tab. 5 b, letzte Spalte). Bei Sn, Sb und Bi führt indessen die Flächengitter-Vorstellung [Nebenmaximum $=(110)$. Flächengitter-Interferenz] zu einfach-kubischen Schichtpaketen ${ }^{72}$ z. Tl. mit Flächengitter-Charakter, dabei ist für $\mathrm{Sn}$ und $\mathrm{Bi}$ die Flächengitter-Kante $a_{\mathrm{Flg}}$. praktisch mit dem kürzesten Atomabstand des zugehörigen Gitters, das sich näherungsweise einfach-kubisch beschreiben läßt, identisch (vgl. Tab. 5 b). Bei Sb liegt die Kantenlänge $a_{\mathrm{Flg}}$, vielleicht wegen der stärkeren Abweichung des Sb-Gitters von der kubischen Struktur, zwischen den ersten beiden Gitterabständen ${ }^{73}$. Eine Deutung der Streukurve von aufgeschmolzenem $\mathrm{Sb}$ und $\mathrm{Bi}$ nach Art von Schichtpaketen mit hexagonaler Gitterzelle und Flächengitter-Charakter wie bei geschmolzenem Ge steht nicht im Einklang mit dem experimentellen Befund. Hier ist offenbar die beobachtete einfach-kubische Struktur als Entzerrung des zugehörigen Gitters zu deuten.

Bei den Schichtpaketen handelt es sich keineswegs um eine feste, stabile Anordnung der Atome, sondern diese Atomgruppierungen bilden sich, je nach den vorliegenden Verhältnissen wie Temperatur-Unterschiede, innere Spannungen usf. als Momentanzustände in kleinsten Bereichen der Schmelze immer wieder von neuem heraus, um gleichzeitig an anderen Stellen zu verschwinden. Durch die homöopolare Bindung begünstigt, kommt es zur Ausbildung von Schichtpaketen; als Gleitebene treten hier die (001) - und mitunter auch die (110). Ebene auf. Die möglichen Flächengitter-Interferenzen sind im letzten Falle (vgl. Anm. ${ }^{50}$, danach muß sein $h=-k$ und $l=$ beliebig): 001, $110,1 \overline{1} 1,002,1 \overline{1} 2$, 220, 003 usf.; d. h. sie liegen dichter beieinander, als wenn die (001) -Ebene Basis-Fläche ist.

Die Intensitätskurve von geschmolzenem In in Abb. $10 \mathrm{~b}$ besitzt bezüglich des 1. Maximums wohl einen schwach unsymmetrischen. aber völlig ungestörten Verlauf, und zwar selbst in der Nähe des Schmelzpunktes (vgl. Gamertsfelder ${ }^{20}$ ). In Abb. $10 \mathrm{~b}$ geben die eingezeichneten Pfeile $\downarrow$ bzw. $\downarrow$ wiederum die Lagen der Flüssigkeits-Maxima bzw. der (100)-Flächengitter-Interferenz an. Die gute Übereinstimmung bezüglich der Interferenz-Lagen zwischen Experiment und Berechnung und die geringfügige Verschiebung des I. FlüssigkeitsMaximums (vgl. Tab. 1) gehen auch aus Tab. 6 hervor. Strukturmäßig gehört In zur unteren Gruppe der Tab. 1, der ungestörte Verlauf seiner Streukurve und die geringe Abweichung zwischen $\operatorname{den}\left(\sin \vartheta_{1} / \lambda\right)$ exp.

72 Aus der Atomverteilungskurve für geschmolzenes Sb schließen Hendus und Müller ${ }^{39}$ ebenfalls auf eine einfach-kuhische (räumliche) Struktur. allerdings mit $a_{\mathrm{kub}}=3.12 \AA$

73 Bei Sb ist: $r_{1}=9.87 \AA . r_{K_{1}}=3,37 \AA$ und $r_{2}=4,27 \AA$; bei $\mathrm{Bi}: r_{1}=3,10 \AA \overline{\mathrm{A}}, r_{\mathrm{K}_{1}}=3,47 \AA$ und $r_{2}=4,53 \AA$. 


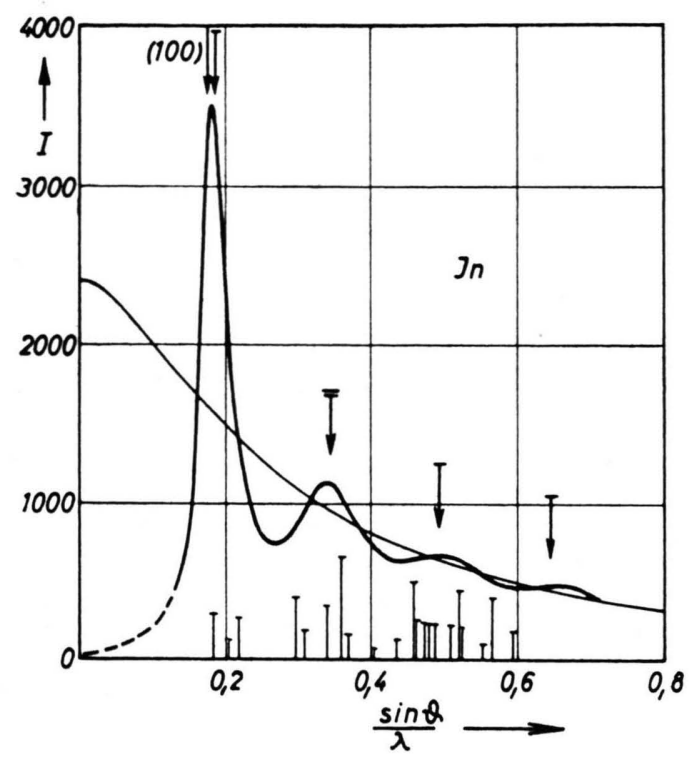

Abb. 10 b. Intensitätskurve von geschmolzenem In.

$\downarrow=$ berechnete Lagen der Flüssigkeits-Maxima,

$\downarrow=$ berechnete Lage der Flächengitter-Interferenz (100).

\begin{tabular}{|c|c|c|c|}
\hline \multicolumn{2}{|c|}{$\begin{array}{c}\left(\frac{\sin \vartheta_{\nu}}{\lambda}\right)_{\text {exp. }} \\
\text { nach GAMERTS- } \\
\text { FELDER }{ }^{20}\end{array}$} & $\begin{array}{c}\left(\frac{\sin \vartheta_{\nu}}{\lambda}\right)_{\text {exp. }} \\
\text { nach GLOCKER } \\
\text { u. HENDUS }{ }^{23}\end{array}$ & $\begin{array}{c}\left(\frac{\sin \vartheta_{\nu}}{\lambda}\right)_{\mathrm{Fl}} . \\
\text { berechnet } \\
\text { aus dem } \\
\text { II. Maximum }\end{array}$ \\
\hline $160^{\circ} \mathrm{C}$ & $390^{\circ} \mathrm{C}$ & $165^{\circ} \mathrm{C}$ & \\
\hline $\begin{array}{l}0,179 \\
0,335 \\
0,50 \\
-\end{array}$ & $\begin{array}{l}0,177 \\
0,335 \\
0,49 \\
-\end{array}$ & $\begin{array}{r}0,181 \\
0,340 \\
0,495 \\
\approx 0,667\end{array}$ & $\begin{array}{l}0,186 \\
0,337_{5} * \\
0,490 \\
0,643\end{array}$ \\
\hline
\end{tabular}

* Berechnung zugrunde gelegter Wert.

Tab. 6. Experimentelle und berechnete Lagen für die Intensitäts-Maxima bei geschmolzenem In.

und $\left(\sin \vartheta_{\mathrm{I}} / \lambda\right)_{\text {ber. }}$-Werten verlangen aber seine Einordnung in die obere Elementen-Gruppe. Die Besprechung der Intensitätskurve von aufgeschmolzenem In erfolgt daher zusammen mit den Elementen der oberen Gruppe.

\section{d) $B l e \mathrm{i}$}

Die Verschiebung des I. Flüssigkeits-Maximums in der Streukurve der unteren Elementen-Gruppe

74 Es sei bemerkt, daß jetzt im Gegensatz zum vorangegangenen einfach-kubischen Falle $a_{\text {hex. }}^{\text {Flg. }}=a_{\mathrm{H}}=r_{1}$ ist mit $r_{1}$ als kürzestem Atomabstand bei dichtester Packung der Atome. Die Berechnung der Lage der Flächengitter-Interferenzen mit der (111) kub.-Ebene als Basis wird wesentlich erleichtert, wenn man beachtet, daß bei hexagonaler Indizierung die (111)-Ebene des flächenzentriert-kubischen Gitters in die $(001)_{\text {hex. }}$-Ebene übergeht. Man hat also in bekannter der Tab. 1 kommt durch die Existenz von Schichtpaketen zustande. Ist eine solche Paketbildung mit z. Tl. Flächengitter-Charakter auch in den Metallschmelzen der oberen Gruppe vorhanden?

In flächenzentriert-kubischen bzw. hexagonalen Gittern ist die (111)- bzw. (001)-Ebene die am dichtesten belegte Ebene. Falls in den Schmelzen dieser Metalle Flächengitter bzw. Schichtpakete vorkommen, dann bauen sich diese sicherlich aus (111) . bzw. (001)-Ebenen z. Tl. mit beliebiger ParallelVerschiebung auf. Hinsichtlich des zu erwartenden Beugungsdiagrammes hat man auch hier wieder $\mathrm{zu}$ unterscheiden zwischen den Raumgitter- und Flächengitter ${ }^{74}$-Interferenzen der Schichtpakete und den Flüssigkeits-Interferenzen des statistischen Kugelmodells. Unter welchen Winkeln treten jetzt diese drei verschiedenen Interferenzen auf? Für die Raumgitter-Interferenz $(111)_{\text {kub. bzw. }}(002)_{\text {hex. }}$ und die Flächengitter-Interferenz $(100)_{\text {hex. }}$ gilt (vgl. auch die Anm. ${ }^{40,50}$ )

$$
\begin{aligned}
& \left(\frac{\sin \vartheta}{\lambda}\right)_{(h k l)}^{\mathrm{Rg} .}=\frac{1}{2 d_{(111) \mathrm{kub} .}}=\frac{1}{2 d_{(002) \mathrm{hex} .}}=\frac{\sqrt{3}}{2 a_{\mathrm{K}}} \\
& =\sqrt{\frac{3}{8}} \frac{1}{a_{\mathrm{H}}}=\sqrt{\frac{3}{8}} \frac{1}{r_{1}}=0,613 \frac{1}{r_{1}} \approx \frac{\sin \vartheta_{\mathrm{I}}}{\lambda}=0,615 \frac{1}{r_{1}}
\end{aligned}
$$

und

$$
\left(\frac{\sin \vartheta}{\lambda}\right)_{(100) \text { hex. }}^{\text {Flg. }}=\sqrt{\frac{1}{3}} \frac{1}{a_{\mathrm{H}}}=\sqrt{\frac{1}{3}} \frac{1}{r_{1}}=0,577 \frac{1}{r_{1}}
$$

mit $a_{\mathrm{K}}=a_{\mathrm{H}} \sqrt{2}$ und $a_{\mathrm{H}}=r_{1}$. Danach fällt die $(002)_{\text {hex }}$-Raumgitter-Interferenz ${ }^{75}$ praktisch mit dem I. Flüssigkeits-Maximum zusammen; die Flächengitter-Interferenz $(100)_{\text {hex. }}$ ist dagegen geringfügig gegenüber der (002) hex-Raumgitter-Interferenz bzw. dem I. Flüssigkeits-Maximum nach der Seite der kleinen $\sin \vartheta / \lambda$-Werte verschoben. Der $A b$ stand der beiden Interferenzen beträgt

$$
\Delta(\sin \vartheta / \lambda)=0,06 \sin \vartheta_{\mathrm{I}} / \lambda .
$$

Für die Winkellage der drei Interferenz-Maxima gilt somit (vgl. die entsprechende Beziehung auf S. 910)

$$
\left(\frac{\sin \vartheta}{\lambda}\right)_{(100)}^{\mathrm{Flg} .}<\left(\frac{\sin \vartheta}{\lambda}\right)_{(002)}^{\mathrm{Rg} .} \approx \frac{\sin \vartheta_{\mathrm{I}}}{\lambda}=0,615 \frac{1}{r_{1}} .
$$

Weise die Lagen der Flächengitter-Interferenzen $(h k 0)$ hex. für die Basisfläche $(001)$ hex. zu bestimmen.

75 Die Raumgitter-Interferenz $(001)_{\text {hex. }}$ ist bei hexagonaler Atomanordnung nicht möglich. Die Raumgitter-Interferen-

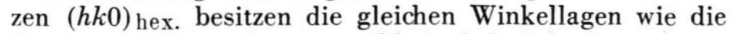
Flächengitter-Interferenzen $(h k 0)$, lediglich die Intensitäts-Verteilung ist verschieden. 


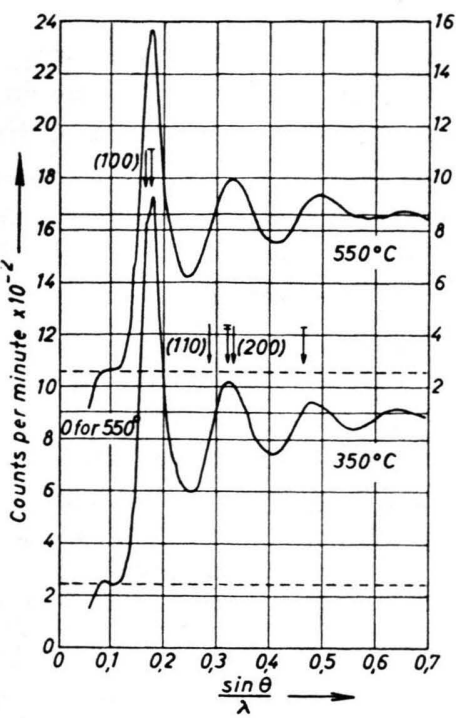

Abb. 11 a. Intensitätskurven von geschmolzenem $\mathrm{Pb}$ bei 350 und $550^{\circ} \mathrm{C}$ (Neutronen-Beugungsaufnahme).

$\downarrow=$ berechnete Lagen der Flüssigkeits-Maxima,

$\downarrow=$ berechnete Lagen der Flächengitter-Interferenzen (100), (110) und (200).

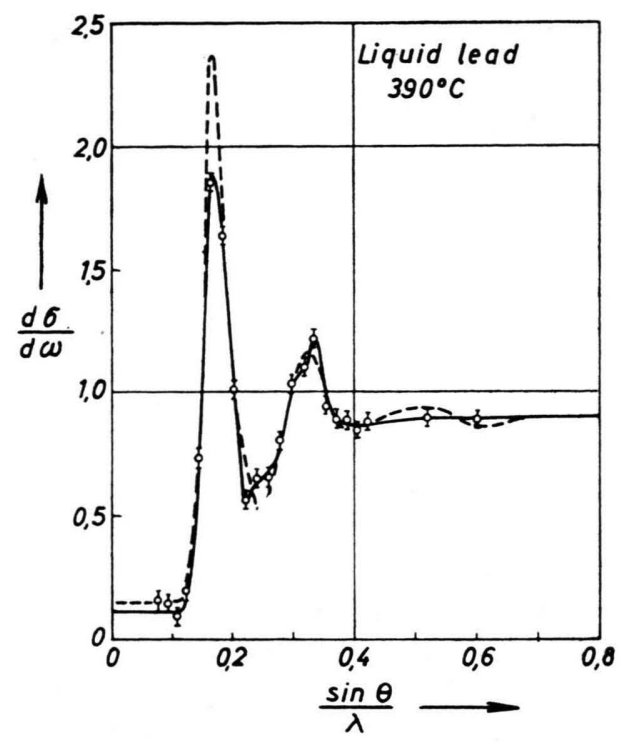

Abb. 11 b. Intensitätskurven von geschmolzenem $\mathrm{Pb}$ bei $390{ }^{\circ} \mathrm{C}$ (Neutronen-Beugungsaufnahme).

\begin{tabular}{|c|c|c|c|c|c|c|}
\hline \multicolumn{6}{|c|}{ Lagen der Maxima in der Intensitätskurve } & \multirow{2}{*}{ 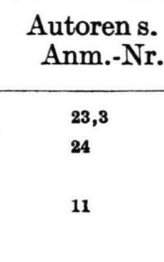 } \\
\hline$\left(\frac{\sin \theta_{\nu}}{\lambda}\right)_{\exp .}$ & $\begin{array}{l}375^{\circ} \mathrm{C} \\
390^{\circ} \mathrm{C}- \\
390^{\circ} \mathrm{C}-- \\
350^{\circ} \mathrm{C} \\
550^{\circ} \mathrm{C}\end{array}$ & $\begin{array}{l}0,174 \\
0,165 \\
0,165 \\
0,180 \\
0,175\end{array}$ & $\begin{array}{l}0,321 \\
0,332 \\
0,321 \\
0,325 \\
0,330\end{array}$ & $\begin{array}{r}0,471 \\
\approx \overline{-} \\
0,514 \\
0,485 \\
0,490\end{array}$ & $\begin{array}{c}\approx 0,607 \\
- \\
\overline{-} \\
0,65 \\
0,65\end{array}$ & \\
\hline \multicolumn{2}{|l|}{$\left(\frac{\sin \theta_{\nu}}{\lambda}\right)_{\text {ber. }}^{\text {Fl. }}$} & 0,176 & 0,321 & 0,465 & 0,609 & \multirow{2}{*}{$\begin{array}{c}\text { Berechnung } \\
\text { mit } \\
r_{1}=3,49\end{array}$} \\
\hline \multicolumn{2}{|c|}{$\left(\frac{\sin \vartheta_{v}}{\lambda}\right)_{(h k 0) \text { ber. }}^{\text {Flg. }}$} & $\begin{array}{l}(100) \\
0,165\end{array}$ & $\begin{array}{l}(110) \\
0,286\end{array}$ & $\begin{array}{l}(200) \\
0,331\end{array}$ & $\begin{array}{l}(210) \\
0,438\end{array}$ & \\
\hline
\end{tabular}

Tab. 7. Experimentelle und berechnete Lagen für die Intensitäts-Maxima und für die Flächengitter-Interferenzen ( $h k 0)$ bei geschmolzenem $\mathrm{Pb}$.

Der Unterschied in den Lagen der InterferenzMaxima ist hier sehr gering (vgl. dagegen die Tab. $2,3,5$ u. a.).

Zur Prüfung des obigen Befundes seien in Abb. 11 a zwei Intensitätskurven von geschmolzenem $\mathrm{Pb}$ (Schmelzpunkt $=327,4{ }^{\circ} \mathrm{C}$ ) betrachtet; sie wurden ebenfalls von Sharrah und Smith ${ }^{11}$ durch Neutronenbeugung mit $\lambda=1,16 \AA$ bei 350 und $550{ }^{\circ} \mathrm{C}$ erhalten. Die eingezeichneten Pfeile $\downarrow$ bzw. $\downarrow$ bedeuten die mit $r_{1}=3,49 \AA$ berechneten InterferenzLagen für die Kugelmodell- bzw. für die Flächengitter-Struktur. Diese Lagen sind in Tab. 7 zusammengestellt. Experiment ${ }^{76}$ und Berechnung stimmen nach Abb. 11. a und Tab. 7 bestens miteinander überein. In der unteren Streukurve $\left(350^{\circ} \mathrm{C}\right.$-Kurve) der Abb. 11 a zeichnet sich links vom 1. Maximum der dichtesten Atompackung durch einen leichten Knick im Kurvenverlauf die Flächengitter-Interferenz (100) ab, und zwar im verlangten Winkelabstand $\Delta(\sin \vartheta / \lambda)=0,06 \sin \vartheta_{\mathrm{I}} / \lambda$ vom 1. Maximum. Auch die (110)-Flächengitter-Interferenz

${ }^{76}$ Die Neutronen-Beugungsaufnahmen der Abb. 8, 11 und 14 d lassen den Flächengitter-Charakter weit deutlicher erkennen als die entsprechenden RöNTGEN-Aufnahmen, sicherlich wegen der Konstanz des Atomformfaktors; zudem sind es Durchstrahlungs-Aufnahmen, die bei weit günstigeren Aufnahme-Bedingungen erhalten wurden. 
bleibt nicht ohne Einfluß auf das Streudiagramm. So zeigt die untere Kurve $\left(350^{\circ} \mathrm{C}\right.$-Kurve $)$ bei einem Vergleich mit der oberen Kurve $\left(550{ }^{\circ} \mathrm{C}\right.$-Kurve $)$ eine allerdings geringfügige Verschiebung des II. Maximums nach kleinen $\sin \vartheta / \lambda$-Werten (vgl. auch Tab. 7). Die Gleitung der Atome führt hier zu Schichtpaketen mit Flächengitter-Charakter und mit der $(111)_{\text {kub.- bzw. }}(001)_{\text {hex. }}$-Ebene als Gleitebene. Mit wachsender Temperatur ist, wie die obere Kurve in Abb. 11 a zeigt, die (100)-FlächengitterInterferenz nicht mehr zu beobachten. Diese Interferenz bleibt aber ähnlich wie bei geschmolzenem $\mathrm{Bi}$ (vgl. Abb. 8 a, obere Kurve) weiterhin im Spiel; sie hebt das I. Flüssigkeits-Maximum zur beobachteten Höhe an. Selbst bei $\mathrm{Pb}$ scheint erst bei Temperaturen weit über dem Schmelzpunkt die Atomverteilung allein durch das statistische Kugelmodell bestimmt zu sein.

Eine Abschätzung des Verhältnisses von Schichtpaket- zu Flüssigkeits-Struktur in geschmolzenem $\mathrm{Pb}$ ist sehr schwierig, und zwar wegen des geringen Winkelabstandes der (100)-Flächengitter-Interferenz vom I. Flüssigkeits-Maximum, ganz besonders aber wegen des Zusammenfallens der Raumgitter-Interferenz (002) der Schichtpakete mit dem I. Flüssigkeits-Maximum und nicht zuletzt wegen der z. Tl. geringen Abweichung der beobachteten Atomzahl ${ }^{77} N_{1}$ von der bei dichtester Kugelpackung (vgl. Tab. 1).

Die Neutronen-Beugungsversuche von ChamberLAIN $^{24}$ an aufgeschmolzenem $\mathrm{Pb}$ bestätigen die obigen Ergebnisse. In Abb. $11 \mathrm{~b}$ sind zwei Streukurven von flüssigem $\mathrm{Pb}$, die bei $390{ }^{\circ} \mathrm{C}$ erhalten wurden, wiedergegeben. Auffallend ist auch hier wie bei flüssigem $\mathrm{Bi}$ der unterschiedliche Kurvenverlauf trotz gleicher Untersuchungs-Temperatur. Die ausgezogene Kurve zeigt bei $\sin \vartheta / \lambda=0,332$ ein recht scharfes Maximum. Aus Tab. 7, in welche die beobachteten und berechneten Lagen der Flüssigkeits-Maxima sowie die der berechneten Flächengitter-Interferenzen $(h k 0)$ eingetragen sind, ersieht man, da $\beta$ das ausgeprägte Maximum der Flächengitter-Interferenz (200) zuzuordnen ist. Die gestrichelte Kurve zeigt indessen bei $\sin \vartheta / \lambda=0,321$ ein breites Maximum, das bei gleicher Winkellage wie bei Hendus ${ }^{3}$ offenbar der Atomanordnung des statistischen Kugelmodells mit $r_{1}=3,49 \AA$ zugeschrieben werden muß. Das 1. Maximum beider Streukurven bei $\sin \vartheta_{1} / \lambda=0,165$

\footnotetext{
77 Wegen der sehr geringen Ausdehnung der Schichtpakete ist die Koordinationszahl $N_{1}$ trotz dichtester Packung der Atome kleiner als 12.
}

stimmt dagegen bezüglich seiner Lage mit der der Flächengitter-Interferenz (100) überein. Bei einem Vergleich der Intensitätskurven von Hendus ${ }^{3}$ und Chamberlain ${ }^{24}$ zeichnet sich bei Chamberlain ${ }^{24}$ der Flächengitter-Charakter am stärksten ab. Hier wird die Lage des 1. Maximums vornehmlich von der (100) -Flächengitter-Interferenz mit $\sin \vartheta / \lambda=0,165$ festgelegt.

Abweichend von der unteren Elementen-Gruppe der Tab. 1 beträgt bei der oberen der Abstand der (100) -Flächengitter-Interferenz vom I. FlüssigkeitsMaximum bzw. von der Raumgitter-Interferenz (002) jeweils etwa $6 \%$ des $\sin \vartheta_{\mathrm{I}} / \lambda$-Wertes. Dabei sind nach S.916 die Lagen dieser drei Interferenzen allein durch den kürzesten Atomabstand $r_{1}=a_{\mathrm{H}}$ festgelegt. Hier läßt sich die Winkellage für die (100)-FlächengitterInterferenz (innerste Grenzlage) leicht angeben, natürlich auch die des I. Flüssigkeits-Maximums (äußerste Grenzlage). Wie die obere Gruppe in Tab. 1 (vgl. Spalte 3 mit 7) zeigt, wird die innerste Grenzlage bei den neueren Untersuchungen an flüssigem $\mathrm{Pb}$ und $\mathrm{Hg}$ erreicht. Nicht ganz so einfach sind die Verhältnisse bezüglich der Lage der verschiedenen Interferenzen bei der unteren Gruppe der Tab. 1. Die unterschiedliche Lage der (100) Flächengitter-Interferenz gegenüber dem I. Flüssigkeits-Maximum ist es, welche die verschieden große Verlagerung des I. Maximums in den Streukurven der beiden Elementen-Gruppen bedingt. Die Verschiebung ist also in beiden Fällen vorhanden, nur ist sie verschieden groß.

Für das Vorliegen von Flächengittern bzw. von Schichtpaketen mit Flächengitter-Charakter sprechen die folgenden Punkte:

1. Auftreten der Flächengitter-Interferenzen (100), (110) u. a., evtl. mit steilem Intensitätsabfall,

2. Verschiebung des I. Flüssigkeits-Maximums,

3. Erniedrigung der Atomzahl $N_{1}$ und

4. Beitrag der Flächengitter-Interferenz (100) zum I. Flüssigkeits-Maximum, insbesondere zu dessen Form, Schärfe und Höhe (vgl. Abb. 14 c).

Punkt 1. entfällt bei zu unterschiedlicher Größe der Flächengitterbereiche sowie bei zu großer Dicke der Schichtpakete. Im letzten Falle bleibt aber bei den Elementen der unteren Gruppe die mitunter recht beträchtliche Verschiebung des I. FlüssigkeitsMaximums wegen des Auftretens der RaumgitterInterferenz (001) bestehen. Bei den Elementen der oberen Gruppe mit ihrer hexagonalen Paket-Struktur scheiden die Kriterien 1 bis 3 von vornherein meistens 


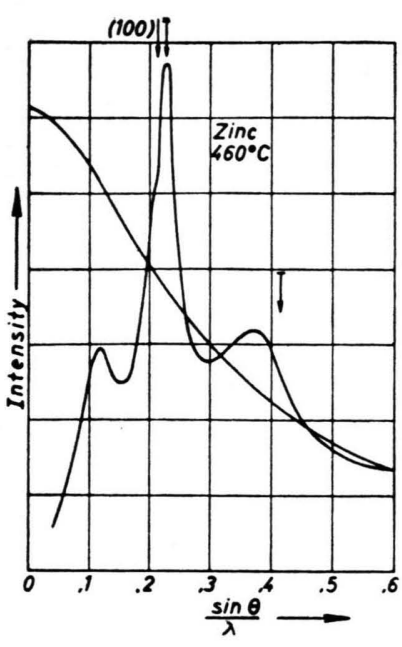

Abb. 12. Intensitätskurve von geschmolzenem $\mathrm{Zn}$. $\mp=$ berechnete Lagen der Flüssigkeits-Maxima, $\downarrow=$ berechnete Lage der Flächengitter-Interferenz (100).

aus. Wohl ist in den Streukurven von flüssigem $\mathrm{Pb}$, $\mathrm{Zn}$ und $\mathrm{Hg}$ (vgl. die Abb. 11 a, untere Kurve, 12 und 14 d) die Flächengitter-Interferenz $(100)_{\text {hex. }}$ noch gut zu erkennen; sobald aber die Auflösung nicht ausreicht ${ }^{78}$ - die Verschiebung des I. FlüssigkeitsMaximums ist hier sehr gering - oder die Schichtpakete von zu unterschiedlicher Größe sind, entfällt die Erkennbarkeit der Flächengitter-Interferenzen. Als einziger Hinweis für das Vorliegen einer Schichtpaket-Bildung bleibt dann die dominierende Höhe und Schärfe des 1. Maximums. Man sieht also, daß es bei den Elementen der oberen Gruppe unvergleichbar schwieriger ist, in deren Schmelzen an Hand ihres Streubildes eine Schichtpaket-Bildung der verlangten Art nachzuweisen.

\section{e) Zink und Cadmium}

In der Streukurve von geschmolzenem $\mathrm{Zn}$ (Schmelzpunkt $=419,4{ }^{\circ} \mathrm{C}$ ), das von GAMERTSFELDER 20 bei $460{ }^{\circ} \mathrm{C}$ untersucht wurde, ist, wie bereits erwähnt, das I. Maximum der dichtesten Atompackung durch OxydBildung kaum gestört (vgl. Tab. 1, Spalte 3 mit 7). Diesem Maximum ist nach Abb. 12 ein kleines Maximum im Abstand $\Delta(\sin \vartheta / \lambda) \approx 0,06 \cdot \sin \vartheta_{\mathrm{I}} / \lambda$ vorgelagert. In diesem Maximum spiegelt sich auch hier die Flächengitter-Struktur der Zn-Schmelze wieder; denn das kleine Maximum ist wie bei flüssigem $\mathrm{Pb}$ in $\mathrm{Abb}$. 11 a als (100)-Flächengitter-Interferenz zu deuten. Danach liegen in der $\mathrm{Zn}$-Schmelze wegen des Abgleitens der Atome auf dichtest belegten Ebenen Schichtpakete

\footnotetext{
78 Vgl. als Beispiel das geschmolzene $\mathrm{Pb}$ nach Hendus ${ }^{3}$.
}

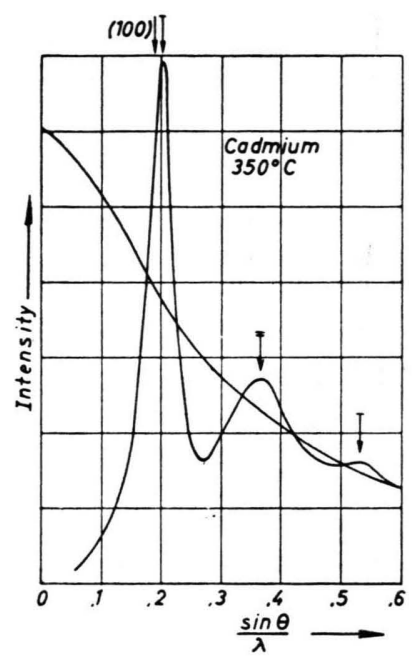

Abb. 13. Intensitätskurve von geschmolzenem Cd.

$\bar{\downarrow}=$ berechnete Lagen der Flüssigkeits-Maxima,

$\downarrow=$ berechnete Lage der Flächengitter-Interferenz (100).

aus (001)-Ebenen mit Flächengitter-Charakter vor. Die in den Abb. 12 und 13 (flüssiges $\mathrm{Cd}$ ) eingezeichneten Pfeile $\downarrow$ bzw. $\downarrow$ geben die Lagen für die FlüssigkeitsMaxima des statistischen Kugelmodells bzw. für die Flächengitter-Interferenz (100) an.

Geschmolzenes Cd (Schmelzpunkt $=321{ }^{\circ} \mathrm{C}$ ) wurde ebenfalls von $\mathrm{G}_{\text {AMERTSFELdER }}{ }^{20}$ bei $350^{\circ} \mathrm{C}$ untersucht. Nach Tab. 1 (vgl. Spalte 3 mit 6 bzw. 7) besteht hier kaum ein Unterschied in den experimentellen und berechneten Werten von $\sin \vartheta_{1} / \lambda$ und $\sin \vartheta_{\mathrm{I}} / \lambda$. Weiter zeichnen sich in der Intensitätskurve der Abb. 13 keinerlei Flächengitter-Interferenzen $a b$; dennoch könnte auch bei Cd wie bei Bi (vgl. Abb. 8 a, obere Kurve) das I. Flüssigkeits-Maximum durch die (100)-Interferenz von Flächengittern mit unterschiedlicher Größe, sowie durch die (002)-Raumgitter-Interferenz von Schichtpaketen mit zu großer Dicke verstärkt sein. Die Höhe dieses Maximums ist etwa von gleicher Größe wie die des I. Maximums von aufgeschmolzenem $\mathrm{Zn}$ mit seiner ausgeprägten Flächengitter-Struktur (vgl. Abb. 12). Ganz ähnlich liegen die Verhältnisse beim geschmolzenen $\mathrm{Tl}$ (Schmelzpunkt $=302,5^{\circ} \mathrm{C}$ ), das von Hendus ${ }^{3}$ bei $375^{\circ} \mathrm{C}$ untersucht wurde (vgl. Tab. 1). Das mitunter beobachtete Fehlen von FlächengitterInterferenzen bei der oberen Gruppe in Tab. 1 schließt ähnlich wie bei Bi (vgl. Abb. 8 a, obere Kurve) bzw. $\mathrm{Sb}$ (vgl. Abb. 9) - hier ist wenigstens noch eine deutliche Überlagerung bzw. Verschiebung des I. Flüssigkeits-Maximums festzustellen - das Vorhandensein einer Paketstruktur selbst mit Flächengitter-Charakter nicht aus.

\section{f) Quecksilber}

Die Ergebnisse einer Reihe von Untersuchungen an flüssigem $\mathrm{Hg}$ sind in Tab. $\mathrm{l}$ aufgeführt. Die Versuche von Richter und HandtManN ${ }^{33}$ an $\mathrm{Hg}$ (Er- 


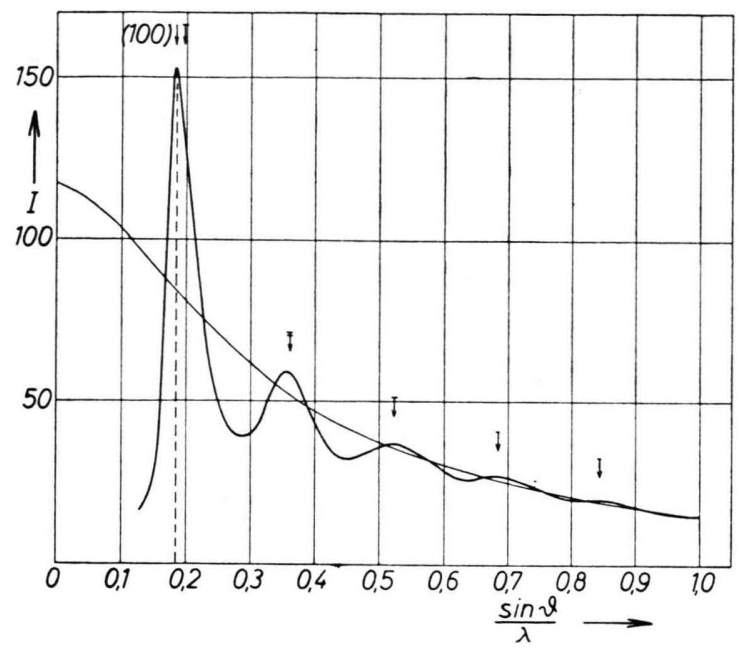

Abb. 14 a. Intensitätskurve von flüssigem $\mathrm{Hg}$.

$\downarrow=$ berechnete Lagen der Flüssigkeits-Maxima,

$\downarrow=$ berechnete Lage der Flächengitter-Interferenz (100).

starrungspunkt $=-38,8{ }^{\circ} \mathrm{C}$ ) wurden bei 18 und $-38{ }^{\circ} \mathrm{C}$ nach dem Zählrohr-Goniometer-Verfahren mit gebogenem Quarzkristall in asymmetrischer Anordnung (vgl. Frohnmeyer und Richter ${ }^{79}$ ) zur Monochromatisierung der verwandten $\mathrm{Cu}$ - und MoStrahlung durchgeführt. Bei beiden Temperaturen wurde die gleiche Streukurve erhalten. Die korrigierte und auf die Atomformfaktor-Werte normierte Intensitätskurve ist in Abb. 14 a wiedergegeben. Die eingezeichneten Pfeile $\downarrow$ bzw. $\downarrow$ markieren die berechneten Lagen für die Flüssigkeits-Maxima bzw. für die Flächengitter-Interferenz (100). Bezüglich der Lage der Flüssigkeits-Maxima, ausgenommen das I. Maximum, besteht eine recht gute Übereinstimmung ${ }^{80}$ zwischen den beobachteten und berechneten Lagen.

Die aus der RöNTGEN-Streukurve unmittelbar und durch Fourier-Analyse erhaltenen charakteristischen Größen sind in Tab. 1 zusammengestellt. Es seien zunächst die aus dem II. Maximum der Intensitätskurve gewonnenen Zahlenwerte betrachtet. Nach oben findet man als kürzesten Atomabstand $r_{1}=3,12 \AA$, in guter Übereinstimmung mit dem Atomradius für Hg bei Zwölfer-Koordination $a_{12}{ }^{*}=1,55 \AA$ A. Für die

79 G. Frohnmeyer u. H. Richter, Naturwiss. 42, 256 [1955].

80 Mitunter beobachtet man, daß die höheren Maxima geringfügig nach größeren $\sin \vartheta / \lambda$-Werten verschoben sind (vgl. die Tab. 2, 3, 6, 7 und 9 a). Dieser Befund ist z. Tl. durch den Atomformfaktor verursacht. Bei Elimination seines Einflusses bleibt die Lage des 1. Maximums praktisch un-

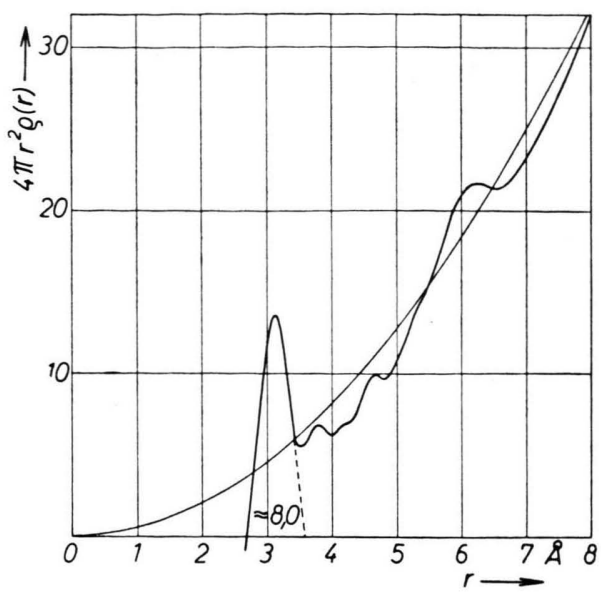

Abb. 14 b. Atomverteilungskurve von flüssigem $\mathrm{Hg}$.

Lage des I. Flüssigkeits-Maximums errechnet man $\left(\sin \vartheta_{\mathrm{I}} / \lambda\right)_{\text {ber. }}=0,197$ (vgl. Tab. 1, Spalte 6), in guter Übereinstimmung mit dem aus der ZwölferKoordination berechneten Wert $\left(\sin \vartheta_{\mathrm{I}} / \lambda\right)_{\text {ber. }}=0,196$ (vgl. Tab. 1, Spalte 7$)$ und mit den $\left(\sin \vartheta_{\mathrm{I}} / \lambda\right)_{\text {ber.- }}$. Werten, wie man sie aus dem II. Maximum der Streukurven anderer Autoren erhält. Das 1. Maximum der Intensitätskurve in Abb. 14 a wird dagegen in Übereinstimmung mit Prins ${ }^{29}$, Hendus ${ }^{32}$, Jennings ${ }^{34}$ sowie Smallman und Frost ${ }^{35}$ bei $\sin \vartheta_{1} / \lambda \approx 0,185$ beobachtet; es ist gegenüber dem aus dem II. Maximum berechneten Wert von $\sin \vartheta_{\mathrm{I}} / \lambda=0,197$ wiederum nach der Seite der kleinen Winkelwerte verschoben. Die Verschiebung beträgt praktisch $6 \%$ des $\sin \vartheta_{\mathrm{I}} / \lambda$-Wertes. Dieses Ergebnis spricht sehr für eine ausgeprägte SchichtpaketStruktur mit Flächengitter-Charakter und dichtester Atompackung in flüssigem $\mathrm{Hg}$. Hierauf deutet auch der steile Abfall des 1. Maximums (vgl. Abb. 14a) nach der Seite der kleinen $\sin \vartheta / \lambda$-Werte hin sowie der allmähliche und damit asymmetrische Intensitätsabfall auf der Gegenseite. Danach liegen im flüssigen $\mathrm{Hg}$ des untersuchten Temperaturbereiches neben der Flüssigkeits-Struktur Schichtpakete aus (001)-Ebenen mit Flächengitter-Charakter vor. Die Zusammenstellung der $\mathrm{Hg}$-Werte in Tab. 1 zeigt, wie mit zunehmender Meßgenauigkeit der aus dem II. Maximum berech-

verändert, dagegen werden die der weiteren Maxima, insbesondere des II. Flüssigkeits-Maximums geringfügig nach größeren Winkelwerten verlagert. Hierdurch wird eine noch bessere Ubereinstimmung zwischen Experiment und Berechnung erreicht. 


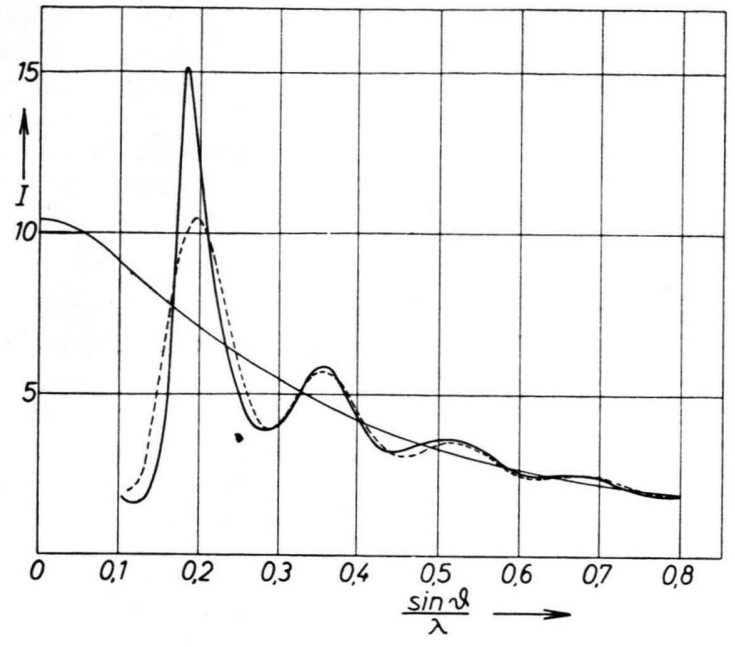

Abb. 14 c. Uberlagerung von Flüssigkeits- und Paket-Struktur im l. Maximum der Intensitätskurve von flüssigem $\mathrm{Hg}$.

nete $\sin \vartheta_{\mathrm{I}} / \lambda$-Wert bzw. der experimentelle Wert von $\sin \vartheta_{1} / \lambda$ immer weniger um den oberen Extremwert $\sin \vartheta_{\mathrm{I}} / \lambda=0,196$ (vgl. Tab. 1, Spalte 6 mit 7 ) bzw. um den unteren Extremwert

$$
\left(\sin \vartheta_{1} / \lambda\right)_{\operatorname{exp.}}^{\mathrm{Min} .}=(\sin \vartheta / \lambda)_{(100)}^{\mathrm{Flg} .}=0,185
$$

streuen (vgl. Spalte 3 und in diesem Zusammenhang auch die $\mathrm{Pb}$-Werte der Tab. 1, Spalte 3, 6 und 7).

Es seien jetzt die Ergebnisse der Fourier-Analyse der Intensitätskurve von flüssigem $\mathrm{Hg}$ nach RichteR und Handtmann ${ }^{33}$ betrachtet. Die Abszisse des ersten Maximums bei $r_{1}=3,12 \AA$ in der Atomverteilungskurve der Abb. 14 b entspricht dem kürzesten Atomabstand in flüssigem $\mathrm{Hg}$. In diesem Abstand findet man etwa 8 Atome vor. Die kleinen, ausgeprägten Maxima bei $r \approx 3,80 \AA$ und $r \approx 4,70 \AA$ sind Nebenmaxima. Bei $r=r_{1} \sqrt{3}=5,40 \AA$ und $r=2 r_{1}=6,24 \AA$ zeichnen sich zwei breite Maxima ab; ihre Abszissenwerte lassen sich mit $r_{1}=a_{\mathrm{H}}=3,12 \AA$ als die Atomabstände eines Sechser-Ringes deuten. In flüssigem $\mathrm{Hg}$ liegen offenbar Schichtpakete mit FlächengitterCharakter und der (111) rhboedr.- bzw. (001) $)_{\text {hex.- }}$ Ebene des Hg-Gitters als Basis vor, allerdings mit $r_{1}=3,12 \AA$ als kürzestem Atomabstand (entsprechender Gitterwert $r_{2}=3,463 \AA$, kürzester Gitterabstand $r_{1}=2,999 \AA$, vgl. Wolf ${ }^{81}$ sowie MeHL und BARRETT ${ }^{82}$ ). Daneben scheint sich in Abb. 14 b auch die

81 M. Wolf, Z. Phys. 53, 72 [1929].

82 R. F. Mehl u. C. S. Barrett, Amer. Inst. Min. Metallurg. Engr. Techn. Publ. Nr. 225 [1929]. - C. S. Barrett, Acta Cryst. 10, 58 [1957].

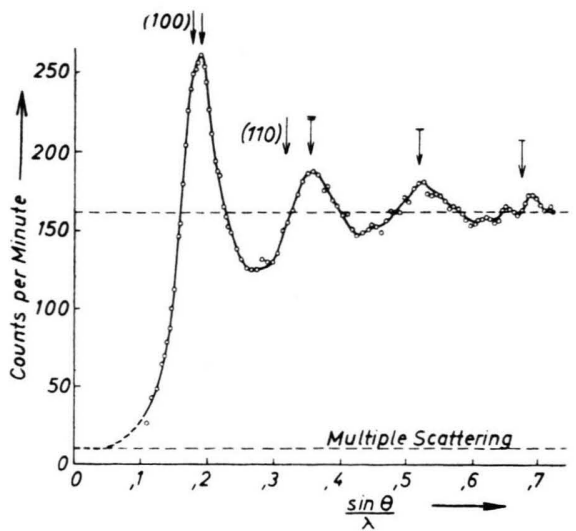

Abb. 14 d. Intensitätskurve von flüssigem $\mathrm{Hg}$ (Neutronen-Beugungsaufnahme).

$\downarrow=$ berechnete Lagen der Flüssigkeits-Maxima,

$\downarrow=$ berechnete Lagen der Flächengitter-Interferenzen (100) und (110).

Beim 1. Maximum hat der rechte Pfeil versehentlich keinen Querstrich.

Atomanordnung des statistischen Kugel-Modells abzuzeichnen, und zwar in einem nur sehr schwach angedeuteten Maximum bei $r=r_{1} \sqrt{2}=4,42 \AA$. Ähnlich liegen die Verhältnisse bei geschmolzenem $\mathrm{Bi}$ (vgl. Sharrah und Smith ${ }^{11}$, Abb. 2 b).

Wie oben ausgeführt wurde, setzt sich die Streukurve einatomiger Metallschmelzen aus den Beiträ. gen der Kugelmodell-Anordnung (Flüssigkeits-Struktur) und der Schichtpakete zusammen. Der Streubeitrag der Schichtpakete beschränkt sich vornehmlich auf das I. Flüssigkeits-Maximum, d. h. der Verlauf der Intensitätskurve außerhalb des I. Maximums wird praktisch von der Kugelmodell-Anordnung, also von der Funktion $\sin k s r_{1} / k s r_{1}$ bestimmt. Berechnet man nach ihr und mit $\left(r_{1}\right)_{\mathrm{Hg}}=3,12 \AA$ in erster Näherung die Streukurve für flüssiges $\mathrm{Hg}$ und gleicht sie nach Berücksichtigung des Temperatur-Faktors bei großen $\sin \vartheta / \lambda$-Werten an die experimentelle Intensitätskurve an, so erhält man das Diagramm der Abb. 14c; hierin bedeutet die ausgezogene Kurve die experimentell gefundene Intensitätsverteilung für flüssiges $\mathrm{Hg}$ und die gestrichelte die berechnete Streukurve für das Kugelmodell. Man sieht, daß das 1. Maximum der Hg-Kurve durch Überlagerung der Streubeiträge von Flüssigkeits- und Paket-Struktur zustande kommt. Dabei ergibt sich der Streuanteil der Paket-Struktur als Differenzbetrag der gestrichelten Kurve von der ausgezogenen Kurve. Außerhalb des 1. Maximums wird der Intensitäts- 
verlauf in sehr guter Übereinstimmung mit dem Experiment allein vom Flüssigkeits-Modell bestimmt. Weiter ist die verschiedene Lage des innersten Maxi-

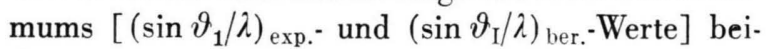
der Streukurven deutlich zu erkennen. Aus dem Unterschied des Flächeninhaltes beider Kurven oberhalb der $\mathrm{F}^{2}$-Kurve und im Bereich des innersten Maximums ermittelt man in erster Näherung als Verhältnis von Paket- zu Flüssigkeits-Struktur den Wert $1: 2$, also einen Wert, der auch hier zeigt, daß die Paket-Struktur einen beachtlichen Anteil in der Schmelze ausmacht.

Als einzige Untersuchung mit Neutronenstrahlen an flüssigem $\mathrm{Hg}$ liegt die von VInEyarD ${ }^{36}$ vor. Die Streukurve in Abb. $14 \mathrm{~d}$ zeigt bei $\sin \vartheta / \lambda \approx 0,184$ ähnlich wie die von flüssigem $\mathrm{Pb}$ (vgl. Abb. $11 \mathrm{a}$, untere Kurve) eine deutliche Störung im Kurvenverlauf, welche durch die (100)-Flächengitter-Interferenz $(\downarrow)$ verursacht ist. Diese Interferenz verlagert das I. Flüssigkeits-Maximum $(\downarrow)$ der dichtesten Atompackung mit $\sin \vartheta_{\mathrm{I}} / \lambda=0,196$ (vgl. Tab. 1, Spalte 7) nach einem kleineren $\sin \vartheta / \lambda$-Wert $\left[\left(\sin \vartheta_{1} / \lambda\right)_{\text {exp. }}=0,194\right]$, während man aus dem II. Maximum in sehr guter Ubereinstimmung mit $\sin \vartheta_{\mathrm{I}} / \lambda=0,196$ den Wert $\left(\sin \vartheta_{\mathrm{I}} / \lambda\right)_{\text {ber. }}=0,198$ erhält. Abb. 14 d stellt damit einen unmittelbaren Beweis für die oben geforderte Struktur des flüssigen $\mathrm{Hg}$ dar.

Das geschmolzene In (Schmelzpunkt $=156,4{ }^{\circ} \mathrm{C}$ ) wurde von $\mathrm{Hendus}^{3}$ und Gamertsfelder ${ }^{20}$ bei $\approx 160$ und $390{ }^{\circ} \mathrm{C}$ untersucht. Die Streukurve von flüssigem In nach Hendus ${ }^{3}$ in Abb. 10 b zeigt bei genauer Betrachtung einen schwach unsymmetrischen Verlauf ähnlich wie die von flüssigem $\mathrm{Hg}$. Diese Unsymmetrie wird nach GAMERTSFELDER ${ }^{20}$ mit steigender Temperatur schwächer. Offenbar hat man es im geschmolzenen In mit einem Nebeneinander von Flüssigkeits- und FlächengitterStruktur zu tun. Der kürzeste (mittlere) Atomabstand $r_{1}=3,32 \AA$, wie man ihn aus den Streukurven von Gamertsfelder ${ }^{20}$ und Hendus ${ }^{3}$ errechnet, ist nach Tab. 1 mit dem aus der Literatur bekannten Atomradius für Zwölfer-Koordination in guter Übereinstimmung. Nach dieser Tabelle ist die Verschiebung des I. Flüssigkeits-Maximums gering $(<6 \%)$. Wegen dieser geringen Verschiebung und des Fehlens von Nebenmaxima gehört das flüssige In zur oberen ElementenGruppe der Tab. 1, d. h. zur Gruppe mit Schichtpaketen mit dichtester Atompackung. Danach. geht beim Aufschmelzen der homöopolare Bindungsanteil des kristallinen In verloren, so daß sich bezüglich der FlüssigkeitsStruktur jedes Atom nach Art der dichtesten Kugelpackung mit der geometrisch größtmöglichen Anzahl von Atomen umgibt. Das gleichzeitige Vorhandensein einer Flächengitter-Struktur setzt aber die beobachtete Atom- zahl auf $N_{1}=8$ bis 9 herab. $\mathrm{Hg}$ und In stehen am Ubergang zwischen den beiden Gruppen der Tab. 1; dabei ist im flüssigen $\mathrm{Hg}$ wegen der größeren Verschiebung des I. Flüssigkeits-Maximums, der größeren Unsymmetrie des 1. Maximums und wegen der kleineren Atomzahl $N_{1}=7$ bis 8 die Flächengitter-Struktur ausgeprägter als beim aufgeschmolzenen In.

\section{g) Alkali-Metalle}

$\mathrm{Zu}$ der oberen Elementen-Gruppe in Tab. 1 gehören auch die Alkali-Metalle. In Abb. 15 ist als Beispiel die Intensitätskurve von geschmolzenem $\mathrm{Na}$ wiedergegeben (Schmelzpunkt $\left.=97,7^{\circ} \mathrm{C}\right)$. Wieder geben die Pfeile $\downarrow$ die berechneten Lagen für die Flüssigkeits-Maxima an. Die Höhe des 1. Maximums ist leicht dominierend. Ein merklicher Unterschied in den Interferenz-Lagen, insbesondere in den $\sin \vartheta_{1} / \lambda$ - bzw. $\sin \vartheta_{\mathrm{I}} / \lambda$-Werten, wie sie vom Experiment und der Berechnung geliefert werden,

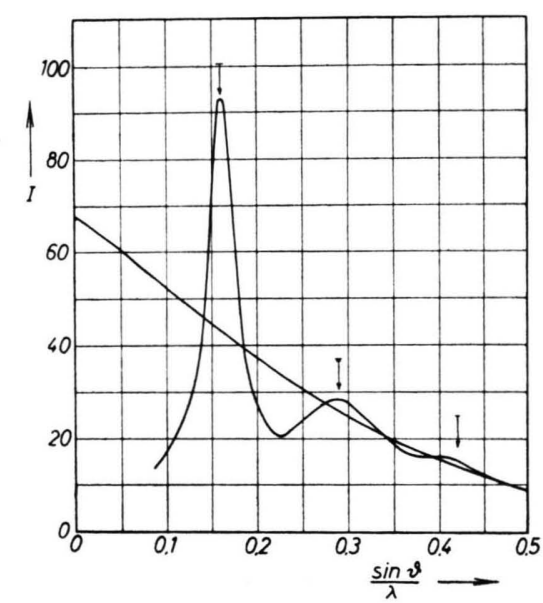

Abb. 15. Intensitätskurve von geschmolzenem Na bei $100^{\circ} \mathrm{C}$. $\bar{\downarrow}=$ berechnete Lagen der Flüssigkeits-Maxima.

ist nach Tab. 1 nicht festzustellen, vielmehr ist hier die Utbereinstimmung überraschend gut. Für das Vorliegen von Flächengitter-Interferenzen bestehen somit keine unmittelbaren Anhaltspunkte. Weitere sehr genaue Intensitätsmessungen sind besonders bezüglich der beobachteten Atomzahlen notwendig.

Wollte man in den Alkali-Schmelzen eine gitterähnliche Anordnung der Atome mit Achter-Koordination zulassen, so ist dieser Fall nach Abb. 7 auch bezüglich einer eventuellen Schichtpaket-Bildung aus (110)-Ebenen mit oder ohne Flächengitter-Charakter auszuschließen. Für das flüssige $\mathrm{Na}$ als Beispiel ist nämlich $(\sin \vartheta / \lambda)_{(100)}^{\text {Flg. }}=0,116$ und nach Anm. ${ }^{40}$ :

$$
(\sin \vartheta / \lambda)_{(110)}^{\mathrm{Flg}}=(\sin \vartheta / \lambda)_{(110)}^{\mathrm{Rg} .} \approx\left(\sin \vartheta_{\mathrm{T}} / \lambda\right)_{\text {ber. }}^{8}=0,164,
$$

nach Tab. 1 ist aber $\left(\sin \vartheta_{\mathrm{I}} / \lambda\right)_{\text {ber. }}^{12}=0,159$.

Nach Abb. 7 besitzen die Alkali-Metalle in ihren Schmelzen eine Zwölfer-Koordination. Der gleiche Be- 


\begin{tabular}{|c|c|c|c|c|}
\hline $\begin{array}{l}\text { Alkali- } \\
\text { Metalle }\end{array}$ & $\left(\frac{\sin \theta_{1}}{\lambda}\right)_{\exp }$ & $\begin{array}{c}\left(\frac{\sin \vartheta_{I}}{\lambda}\right)_{\text {ber. }} . \\
\text { aus-dem } \\
\text { II. Max. }\end{array}$ & $\begin{array}{c}\left(\frac{\sin \vartheta_{\mathrm{I}}}{\lambda}\right)_{\mathrm{ber}} . \\
\text { für 12er- } \\
\text { Koord. }\end{array}$ & $\begin{array}{c}\left(\frac{\sin \vartheta_{\mathrm{I}}}{\lambda}\right)_{\text {ber. }} \text {. } \\
\text { für 8er- } \\
\text { Koord. }\end{array}$ \\
\hline $\mathrm{Li}$ & 0,195 & 0,195 & 0,195 & 0,201 \\
\hline $\mathrm{Na}$ & $\begin{array}{l}0,155 \\
0,161\end{array}$ & $\begin{array}{l}0,155 \\
0,160\end{array}$ & 0,159 & 0,164 \\
\hline $\mathbf{K}$ & $\begin{array}{l}0,129 \\
0,130\end{array}$ & $0, \overline{129}$ & 0,129 & 0,133 \\
\hline $\mathrm{Rb}$ & 0,122 & - & 0,121 & 0,126 \\
\hline $\mathrm{Cs}$ & 0,111 & - & 0,112 & 0,117 \\
\hline
\end{tabular}

Tab. 8. Vergleich der experimentellen bzw. der aus dem II. Maximum berechneten Werte von $\left(\sin \vartheta_{1} / \lambda\right)$ exp. bzw. $\left(\sin \vartheta_{\mathrm{I}} / \lambda\right)_{\text {ber. }}^{\text {II.Max. }}$ mit den $\left(\sin \vartheta_{\mathrm{I}} / \lambda\right)$ ber.-Werten für Zwölferund Achter-Koordination.

fund geht auch aus Tab. 8 hervor. Hier sind die berechneten $\sin \vartheta_{\mathrm{I}} / \lambda$-Werte ${ }^{83}$ für Zwölfer- und AchterKoordination neben den experimentellen Werten von $\sin \vartheta_{1} / \lambda$ und den aus dem II. Maximum berechneten $\sin \vartheta_{\mathrm{I}} / \lambda$-Werten (vgl. Tab. 1) eingetragen. Abgesehen von dem älteren Wert von Randall und Rooksby 25 für $\mathrm{Na}$ mit $\sin \vartheta_{1} / \lambda=0,166$ sind die $\left(\sin \vartheta_{\mathrm{I}} / \lambda\right)_{\text {ber. }}$ Werte für Zwölfer-Koordination mit den Werten von

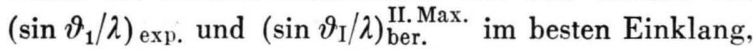
während die der Achter-Koordination eine starke Abweichung zwischen Experiment und Berechnung erkennen lassen; sie liegen insgesamt zu hoch. Mit Tab. 8 ist das Ergebnis der Abb. 7 wenigstens für die AlkaliMetalle bezüglich beider Koordinationen auch zahlenmäßig belegt.

Vergleicht man die Volumen-Änderung $\Delta V$ der Elemente am Schmelzpunkt miteinander, so stellt man fest, daß diese Änderung bei den Alkali-Metallen Li, Na usf. ( $\Delta V=1,5$ bis $2,5 \%$ ) besonders gering ist im Gegensatz $\mathrm{zu}$ der bei den ausgesprochenen Metallen wie $\mathrm{Au}, \mathrm{Ag}, \mathrm{Cu}$ u. a. mit $\Delta V=5,2$ bis $4,1 \%$ (vgl. Borelius ${ }^{84}$ sowie $\mathrm{U}_{\text {BвELOHDE }}{ }^{85}$ ). Die geringe Volumen-Änderung $\Delta V$ bei den Alkali-Metallen erklärt sich durch den Übergang von der Achter- zur Zwölfer-Koordination beim Schmelzvorgang.

\section{h) Gold und Aluminium}

Als einzige Untersuchung an aufgeschmolzenem $\mathrm{Au}$ (Schmelzpunkt $=1063^{\circ} \mathrm{C}$ ) liegt die von Hennus ${ }^{3}$ vor. Hendus hat das flüssige $\mathrm{Au}$ bei etwa $1100^{\circ} \mathrm{C}$ untersucht. Die Lage des 1. Maximums von geschmolzenem $\mathrm{Au}$ in Abb. 6 ist gegenüber dem aus dem II. Flüssig. keits-Maximum berechneten $\sin \vartheta_{\mathrm{T}} / \lambda$-Wert geringfügig nach kleinen Winkelwerten verschoben (vgl. Tab. 1, Spalte 3 mit 6). Auch bei Al ist der experimentelle Wert von $\sin \vartheta_{1} / \lambda$ etwas kleiner als der aus dem

83 Die Berechnung erfolgte in beiden Fällen nach der Gleichung $\sin \vartheta_{\mathrm{T}} / \lambda=0,306 \cdot 1 / a_{\mathrm{K}}^{*}$, wobei für $a_{\mathrm{K}}{ }^{*}$ der Wert des Atomradius für Zwölfer- bzw. Achter-Koordination einzusetzen ist.
Atomradius $a_{12}{ }^{*}$ für Zwölfer-Koordination berechnete Wert (vgl. Tab. 1, Spalte 3 mit 7). Obwohl die Streukurven von flüssigem $\mathrm{Au}$ und $\mathrm{Al}$ keinerlei Anzeichen für das Vorliegen von Flächengitter-Interferenzen unmittelbar erkennen lassen, könnte dennoch die obige Verschiebung als Hinweis für das Vorliegen einer Paket-Struktur gedeutet werden; vor allem spricht auch hierfür die beherrschende Höhe des 1. Maximums in Abb. 6 und nicht zuletzt der eingangs diskutierte Vergleich von experimenteller und berechneter Atomverteilung und da besonders die fehlende zweite Atomgruppe des statistischen Kugelmodells bei $r_{2}=r_{1} \sqrt{2}$ (vgl. aber $\mathrm{Bi}$ und $\mathrm{Hg}$ ). Allerdings wäre es auch denkbar, daß hier die Streuungen der Atome der zweiten Sphäre der Kugelmodell-Anordnung bereits so groß sind, daß sich selbst diese Atomgruppe in der Dichteverteilungskurve nicht abzuzeichnen vermag. Wie auch immer die Dinge liegen mögen, die Tendenz zur Schicht- bzw. PaketBildung dürfte in geschmolzenem Au gering sein, ebenso der zugehörige Temperaturbereich; er wird sich auf die nächste Nachbarschaft des Schmelzpunktes beschränken.

\section{i) Argon und Helium}

Der Einfluß der Temperatur auf den Verlauf der Streukurve ist für das flüssige $\mathrm{Ar}$ aus $\mathrm{Abb} .16 \mathrm{a} \mathrm{zu}$ ersehen. Hier sind eine ganze Reihe von Intensitätskurven wiedergegeben, wie sie von Eisensters und Gingrich ${ }^{19}$ mittels Röntgen-Strahlen unterhalb der kritischen Temperatur (untersuchter Temperaturbereich 84,4 bis $149,3^{\circ} \mathrm{K}$ ) und des kritischen Druckes erhalten wurden $\left(T_{\mathrm{kr} .}=155,66^{\circ} \mathrm{K}\right.$ und $\left.p_{\mathrm{kr} .}=47,66 \mathrm{Atm}\right)$. Man sieht an der Kurve (1) [oberste Kurve] und an Tab. 9 a, daß die Übereinstimmung zwischen den experimentellen und berechneten $(\downarrow)$ Lagen der Flüssigkeits-Maxima sehr gut ist. Der Berechnung wurde als kürzester Atomabstand einmal der experimentelle Wert $\left(r_{1}\right)_{\text {exp. }}=3,79 \AA$ und das andere Mal der aus dem I. Maximum berechnete Wert $\left(r_{1}\right)_{\text {ber. }}=3.87 \AA \AA$ zugrunde gelegt. Der Verlauf der Streukurven dürfte selbst bei den tiefsten Temperaturen wohl allein vom FlüssigkeitsModell bestimmt sein. Weiter erkennt man aus Abb. 16 a, wie mit Annäherung an die Siedetemperatur die Maxima breiter und flacher werden, ein Zeichen dafür, daß die Streuung der Atomlagen stetig zu- und die Ordnung abnimmt. ebenso nimmt die Dichte und mit ihr die Atomzahl $N_{1}$ ab, und zwar nach $\mathrm{Abb} .16 \mathrm{~b}$ von $N_{1}=10,6$ [Kurve (1)] auf $N_{1} \approx 4.0$ [Kurve (5)]. Die Verschiebung des I. Flüssigkeits-Maximums mit der Temperatur ist hier durch die Vergrößerung des kürzesten Atomabstandes bedingt. In Tab. $9 \mathrm{~b}$ sind die von Eisenstein und Gingrich ${ }^{19}$ angegebenen kürzesten

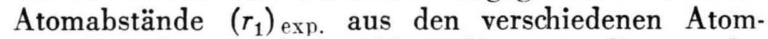
verteilungskurven (vgl. Abb. 16 b) sowie die aus der Lage des I. und II. Flüssigkeits-Maximums berechne-

84 G. Borelius, Hdb. d. Metallphys. I, 231 [1935].

85 A. R. Ubbelohde, An Introduction to Modern Thermodynamical Principles, Clarendon Press, Oxford 1952. 


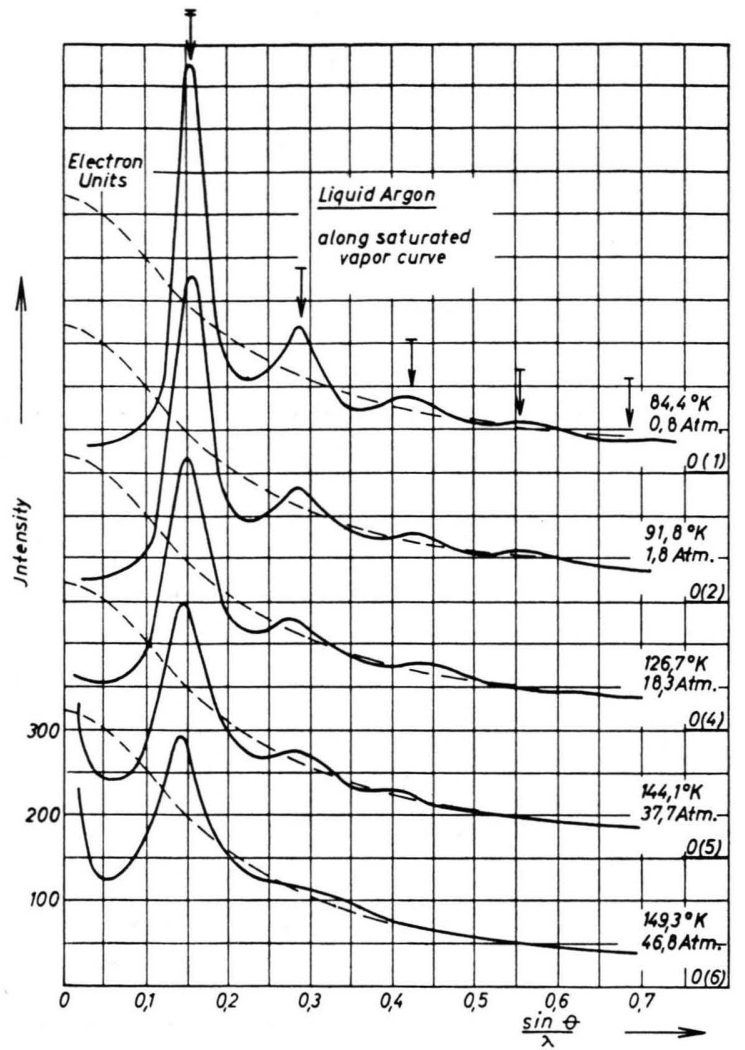

Abb. 16 a. Intensitätskurven von flüssigem Ar bei verschiedenen Temperaturen und Drucken.

$\downarrow=$ berechnete Lagen der Flüssigkeits-Maxima,

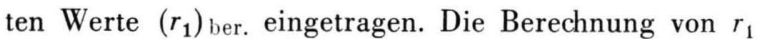
ist nur so lange möglich, als die Lagen der Maxima allein von diesem Abstand bestimmt werden. Die Übereinstimmung entsprechender berechneter $\left(r_{1}\right)_{\text {ber-. }}$-Werte untereinander ist recht gut, mit den experimentellen dagegen weniger. Weiter sieht man, wie mit wachsender Temperatur der kürzeste Atomabstand von $\left(r_{1}\right)_{\text {ber. }}=$ $3,87 \AA$ [Kurve (1)] auf $\left(r_{1}\right)_{\text {ber. }}=4,27 \AA$ [Kurve (6)] ansteigt. Zum Vergleich hierzu sei bemerkt, daß der kürzeste Atomabstand im Ar-Gitter ${ }^{86}\left[\left(a_{\mathrm{K}}\right)_{\text {flzk. }}=5,44 \AA\right.$, vgl. Winkler $\left.{ }^{48}\right] \quad r_{1}=3,84 \AA$ A beträgt. Henshaw, Hurst und Pope ${ }^{19}$ haben das flüssige Ar bei $86,3{ }^{\circ} \mathrm{K}$ mit Neutronenstrahlen der Wellenlänge $\lambda=1,08 \AA$ untersucht. Nach Tab. 1 besteht eine recht gute Übereinstim. mung zwischen den Ergebnissen der RöNtgen-StrahlenBeugung von Eisenstein und Gingrich ${ }^{19}$ und den Resultaten der Neutronen-Beugung.

Das flüssige $\mathrm{He}$ wurde von Reerie und Hutchison ${ }^{16}$ im Temperaturbereich von 1,27 bis $4,20{ }^{\circ} \mathrm{K}$ mit $\mathrm{CuK}_{a^{-}}$ Strahlung untersucht. Die Autoren geben als Lagen für das I. und II. Flüssigkeits-Maximum $2 \vartheta_{1}=28,5^{\circ}$ und $2 \vartheta_{2}=55^{\circ}$ sowie als kürzesten Atomabstand $r_{1} \approx 3,25 \AA$

86 Vgl. auch Landolt-Börnstein, I. Bd., 4. Tl., S. 521 usf., Springer-Verlag, Berlin 1955.

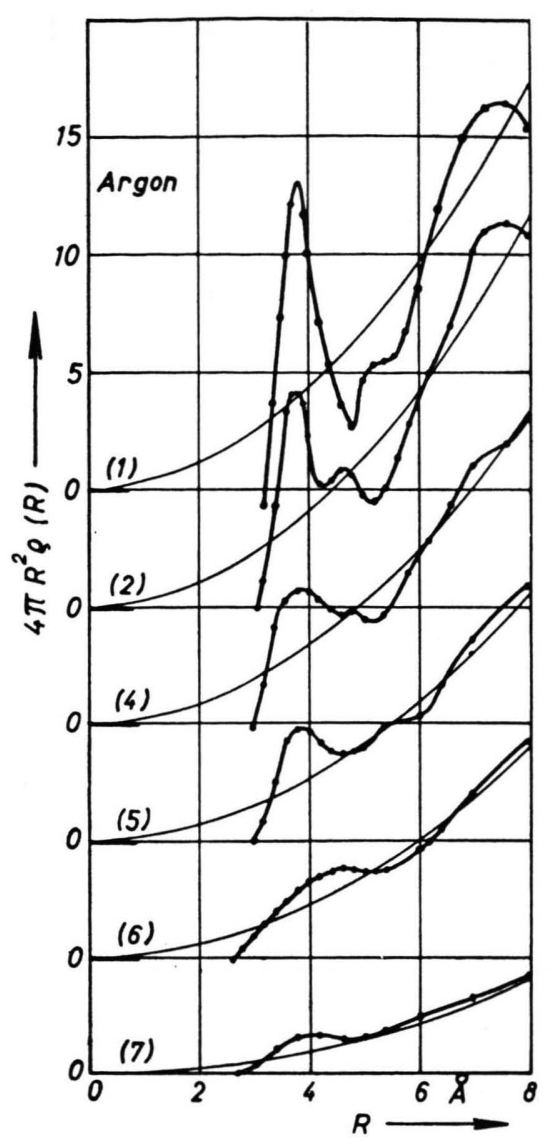

Abb. 16 b. Atomverteilungskurven von flüssigem Ar, Kurve $(7)=$ Verteilungs-Kurve von gasförmigem Ar.

an. Rechnerisch findet man aber (vgl. Tab. 1 bzw. Abb. 7) $r_{1}=3,73 \AA$, etwa in Übereinstimmung mit $r_{1}=a_{\mathrm{H}}=3,61 \AA$ für das kristalline He. Ebenfalls mit $\mathrm{CuK}_{\alpha}$-Strahlung haben Gordon, SHaw und DaunT ${ }^{17}$ das flüssige He bei 1,4 und $4,2{ }^{\circ} \mathrm{K}$ untersucht. Die Lage für das 1. Maximum von flüssigem $\mathrm{He}$ bei $4,2{ }^{\circ} \mathrm{K}$ und der daraus berechnete kürzeste Atomabstand $\left[\left(r_{1}\right)_{\text {ber. }}=\right.$ $3,78 \AA$ A ] sind in Tab. 1 aufgeführt. Hurst und Henshaw ${ }^{17}$ untersuchten dagegen das flüssige $\mathrm{He}$ bei 1,65 bis $5,04{ }^{\circ} \mathrm{K}$ mit Neutronenstrahlen der Wellenlänge $\lambda=1,04 \AA$ A. Aus der angegebenen Lage für das 1. Intensitäts-Maximum $\left(2 \vartheta_{1}=19,6 \pm 0,5^{\circ}\right)$ errechnet man als kürzesten Atomabstand $r_{1}=3,74 \pm 0,10 \AA \AA$, der nach Tab. 1 mit dem Ergebnis der Fourier-Analyse $r_{1}=3,70 \AA$ 践 gut übereinstimmt. Nach diesen Arbeiten wird die Atomanordnung in flüssigem $\mathrm{He}$ am besten durch eine Zwölfer-Koordination beschrieben.

Zusammenfassend läßt sich sagen, daß die Vorstellung von dem Vorhandensein von Schichtpaketen in einatomigen Metallschmelzen bei einer Reihe von 


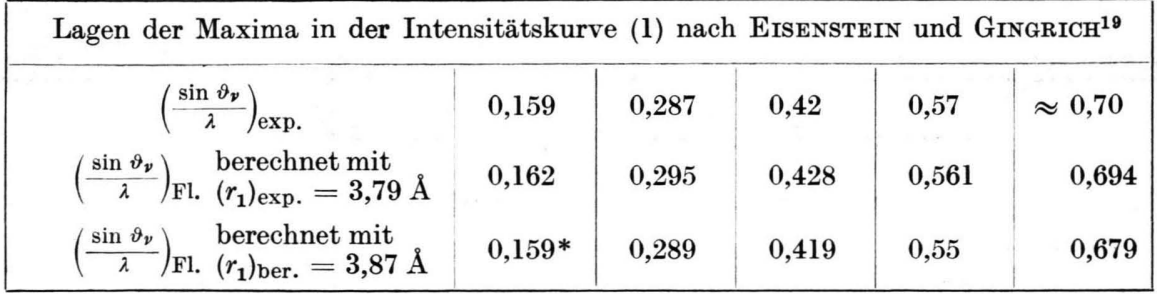

* Berechnung zugrunde gelegter Wert.

Tab. 9 a. Experimentelle und berechnete Lagen für die Intensitäts-Maxima bei flüssigem Ar.

\begin{tabular}{|c|l|l|l|l|l|l|}
\hline $\begin{array}{c}\text { Streu- u. Vert.-Kurven nach } \\
\text { EisEnStEIN uns GINGRICH }\end{array}$ & $(1)$ & $(2)$ & $(4)$ & $(5)$ & $(6)$ & $(7)$ \\
\hline$\left(r_{1}\right)_{\text {exp. }}$ & 3,79 & 3,79 & 3,80 & 3,80 & 4,50 & 4,10 \\
$\left(\frac{\sin \vartheta_{1}}{\lambda}\right)_{\text {exp. }} \approx \frac{\sin \vartheta_{1}}{\lambda}$ & 0,159 & 0,158 & 0,153 & 0,149 & 0,144 & 0,140 \\
$\left(r_{1}\right)_{\text {ber. }}$ & 3,87 & 3,89 & 4,02 & 4,13 & 4,27 & 4,39 \\
$\left(\frac{\sin \vartheta_{2}}{\lambda}\right)_{\text {exp. }} \approx \frac{\sin \vartheta_{I I}}{\lambda}$ & 0,287 & 0,288 & 0,277 & 0,28 & - & - \\
$\left(r_{1}\right)_{\text {ber. }}$ & 3,90 & 3,89 & 4,04 & 4,00 & - & - \\
\hline
\end{tabular}

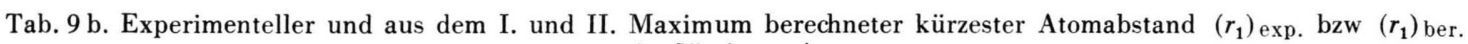
in flüssigem Ar.

Elementen bestätigt werden konnte, besonders in der Nähe des Schmelzpunktes. Die Schmelze gewisser Elemente erweist sich also als ein System von Schichtpaketen, die in dauernder Entstehung und Auflösung sowie im Strukturaustausch mit dem Kugelmodell der dichtesten Atompackung begriffen sind. Bei Temperaturen über dem Schmelzpunkt wird der Verlauf der Streukurve früher oder später allein vom Kugelmodell bestimmt, wo die Atome nach Art der dichtesten Kugelpackung statistisch angeordnet sind. Man sieht also, daß das Streudiagramm selbst von einfachen Metallschmelzen recht komplexer Natur sein kann. Aus diesem Grunde ist es angezeigt, bei der Wahl eines Struktur-Modells auch die Ergebnisse aus der Diskussion der Streukurve mit heranzuziehen.

87 H. RichteR, Z. Naturforschg. 13 a, [1958], im Erscheinen. H. Richter u. G. Gommel, Z. Naturforschg. 12 [1957], im Erscheinen.
Die vorstehenden Überlegungen hinsichtlich der Berechnung der Interferenz-Lagen lassen sich auf den festen amorphen Körper übertragen. Während bei den einatomigen Metallschmelzen im wesentlichen der kürzeste Atomabstand den Verlauf der Streukurve bestimmt, wird ihr Verlauf bei den festen amorphen Stoffen vornehmlich von den $\mathrm{Ab}$ messungen des Grundbausteines, also meistens von zwei Atomabständen, festgelegt (vgl. Richter ${ }^{87}$ ), d. h. der Grundbaustein zeichnet sich nicht allein in der Atomverteilungskurve, sondern bereits in der Intensitätskurve mit aller Deutlichkeit ab.

Der Deutschen Forschungsgemeinschaft sei für vielseitige Unterstützung dieser Untersuchungen bestens gedankt. 